\title{
Optical Adhesive Property Study
}

\author{
Kansas City Division
}

P. D. Sundvold

KCP-613-5631

Published January 1996

Topical Report

Approved for public release; distribution is unlimited.

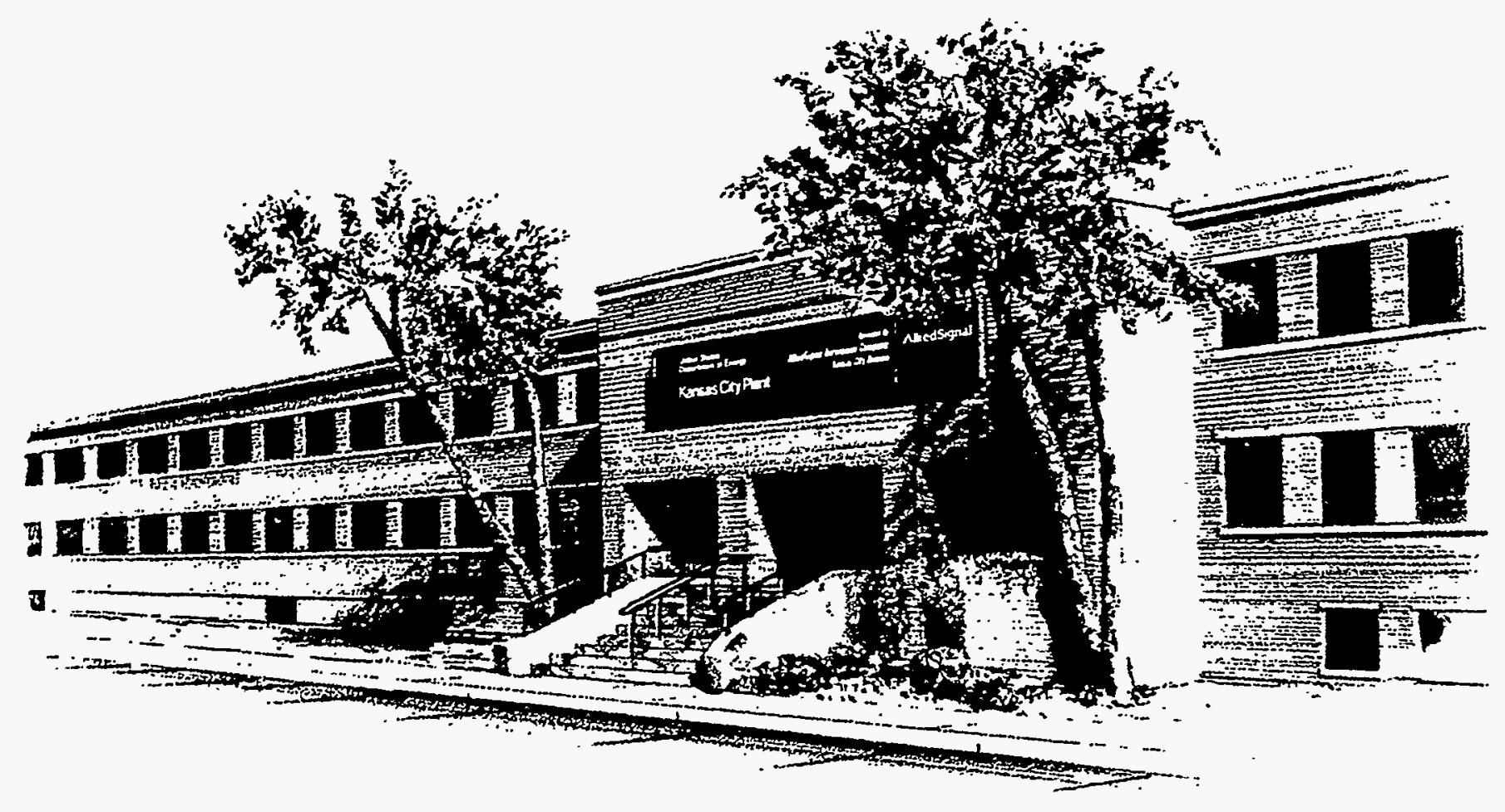




\section{DISCLAIMER}

This report was prepared as an account of work sponsored by an agency of the United States Government. Neither the United States Government nor any agency thereof, nor any of their employees, makes any warranty, express or implied, or assumes any legal liability or responsibility for the accuracy, completeness, or usefulness of any information, apparatus, product, or process disclosed, or represents that its use would not infringe privately owned rights. Reference herein to any specific commercial product, process, or service by trade names, trademark, manufacturer, or otherwise, does not necessarily constitute or imply its endorsement, recommendation, or favoring by the United States Government or any agency thereof. The views and opinions of authors expressed herein do not necessarily state or reflect those of the United States Government or any agency thereof.

All data prepared, analyzed and presented has been developed in a specific context of work and was prepared for internal evaluation and use pursuant to that work authorized under the referenced contract. Reference herein to any specific commercial product, process or service by trade name, trademark, manufacturer, or otherwise, does not necessarily constitute or imply its endorsement, recommendation, or favoring by the United States Government, any agency thereof or AlliedSignal Inc.

Printed in the United States of America.

This report has been reproduced from the best available copy.

Available to DOE and DOE contractors from the Office of Scientific and Technical Information, P. O. Box 62, Oak Ridge, Tennessee 37831; prices available from (615) 576-8401, FTS 626-8401.

Available to the public from the National Technical Information Service, U. S. Department of Commerce, 5285 Port Royal Rd., Springfield, Virginia 22161.

A prime contractor with the United States Department of Energy under Contract Number DE-ACO4-76-DP00613.
AlliedSignal Inc. Kansas City Division P. O. Box 419159 Kansas City, Missouri 64141-6159 
KCP-613-5631

Distribution Category UC-706

Approved for public release; distribution is unlimited.

\title{
OPTICAL ADHESIVE PROPERTY STUDY
}

\author{
P. D. Sundvold
}

Published January 1996

Topical Report

P. D. Sundvold, Project Leader

Project Team:

B. G. Parker

B. D. Sparrow 


\section{Contents}

Section

Page

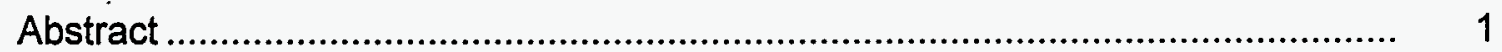

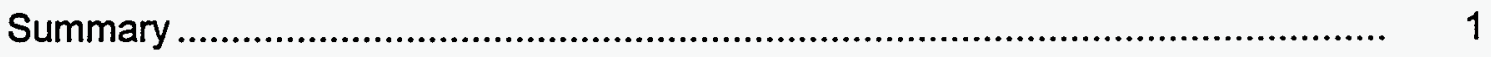

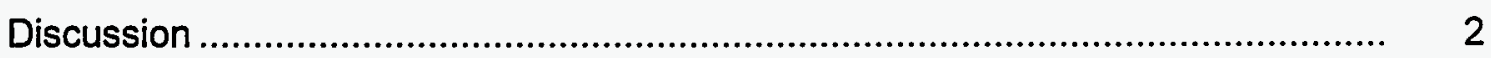

Scope and Purpose.................................................................................... 2

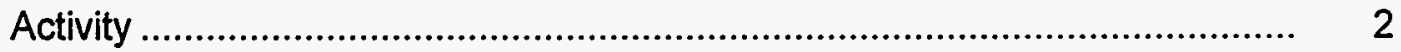

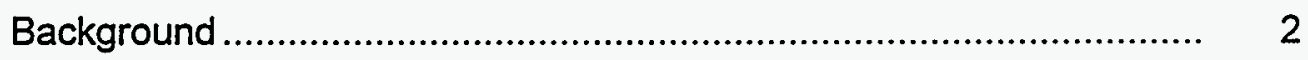

Results .....................................................................................

Accomplishments ...................................................................................

Future Work ....................................................................................... 5

Appendices

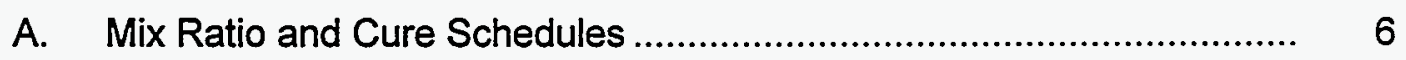

B. Tensile Strength ........................................................................ 8

C. Compressive Strength ....................................................................... 11

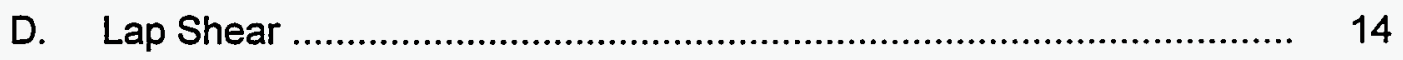

E. Coefficient of Thermal Expansion..................................................... 17

F. RMS Glass Transition Temperature ............................................. $\quad 30$

G. DMA Glass Transition Temperature ................................................. 53

H. Thermal Stability........................................................................... 61

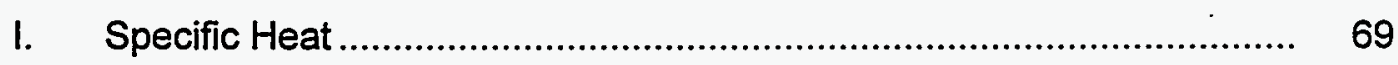

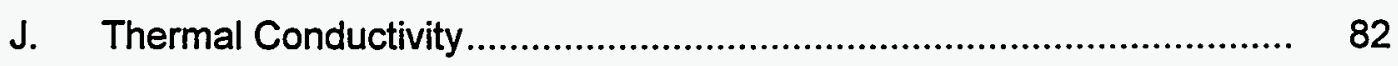

K. Shrinkage and Density .................................................................... 91

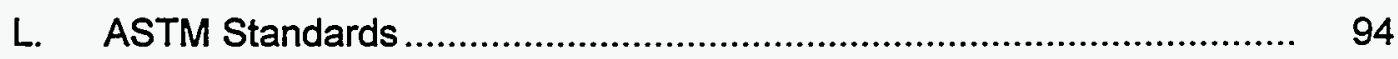




\section{Illustrations}

Figure

Page

E-1 Dimensional Change as a Function of Temperature for A12 1:1 Ratio

E-2 Dimensional Change as a Function of Temperature for A12 1:2 Ratio

E-3 Dimensional Change as a Function of Temperature for A12 2:3 Ratio

E-4 Dimensional Change as a Function of Temperature for EC2216

E-5 Dimensional Change as a Function of Temperature for EA9309.3 NA.

E-6 Dimensional Change During Temperature Cycle for A12 1:1 Ratio............... 25

E-7 Dimensional Change During Temperature Cycle for A12 1:2 Ratio............... 26

E-8 Dimensional Change During Temperature Cycle for A12 2:3 Ratio............... 27

E-9 Dimensional Change During Temperature Cycle for EC2216 ..................... 28

E-10 Dimensional Change During Temperature Cycle for EA9309.3 NA ............... 29

F-1 Shear Modulus as a Function of Temperature for A12 1:1 Ratio................... 33

F-2 Shear Modulus as a Function of Temperature for A12 1:2 Ratio................... 34

F-3 Shear Modulus as a Function of Temperature for A12 2:3 Ratio................... 35

F-4 Shear Modulus as a Function of Temperature for EC2216 ......................... 36

F-5 Shear Modulus as a Function of Temperature for EA9309.3 NA................. 37

G-1 Frequency Response as a Function of Temperature for A12 1:1 Ratio

G-2 Frequency Response as a Function of Temperature for A12 1:2 Ratio

G-3 Frequency Response as a Function of Temperature for A12 2:3 Ratio

G-4 Frequency Response as a Function of Temperature for EC2216. 
G-5 Frequency Response as a Function of Temperature for EA9309.3 NA

$\mathrm{H}-1$ Percent Weight as a Function of Temperature for A12 1:1 Ratio

H-2 Percent Weight as a Function of Temperature for A12 1:2 Ratio ................. 65

H-3 Percent Weight as a Function of Temperature for A12 2:3 Ratio ................ 66

H-4 Percent Weight as a Function of Temperature for EC2216 ..................... 67

H-5 Percent Weight as a Function of Temperature for EA9309.3 NA ............... 68

1-1 Specific Heat as a Function of Temperature for the Optical Adhesives

I-2 Heat Flow as a Function of Temperature at $2^{\circ} \mathrm{C} / \mathrm{min}$ for EC2216................ 74

I-3 Heat Flow as a Function of Temperature at $4^{\circ} \mathrm{C} / \mathrm{min}$ for EC2216.............. 75

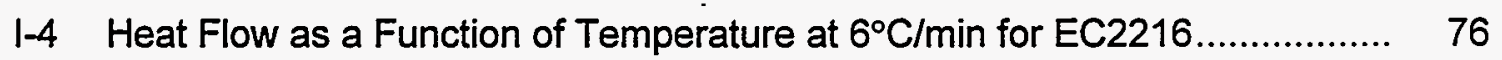

I-5 Heat Flow as a Function of Temperature at $8^{\circ} \mathrm{C} / \mathrm{min}$ for EC2216 ............. 77

I-6 Heat Flow as a Function of Temperature at $10^{\circ} \mathrm{C} / \mathrm{min}$ for $\mathrm{EC} 2216 \ldots \ldots \ldots \ldots \ldots .78$

1-7 Log of the Heat Rate Versus the Reciprocal of the Temperature for EC2216.................................................................................. 79

I-8 Predicted Cure Rate at $71^{\circ} \mathrm{C}$ and $22^{\circ} \mathrm{C}$ for EC2216 ............................ 80

1-9 Predicted Cure Rate at $22^{\circ} \mathrm{C}$ and Measured Value for EC2216 ............... 81

$\mathrm{J}-1$ Thermal Conductivity as a Function of Temperature for A12 1:1.............. 85

$\mathrm{J}-2$ Thermal Conductivity as a Function of Temperature for A12 1:2 .............. 86

J-3 Thermal Conductivity as a Function of Temperature for A12 2:3............... 87

J-4 Thermal Conductivity as a Function of Temperature for EC2216 ............. 88

J-5 Thermal Conductivity as a Function of Temperature for EA9309.3 NA ............................................................................... 89

J-6 Pyrocerm 9606 Reference Standard ................................................ 90 


\section{Tables}

Number $\quad$ Page

1 Summary of Testing Methods ...................................................................

2 Properties of EC2216 .....................................................................

A-1 Mix Ratio and Cure Schedule................................................................... 7

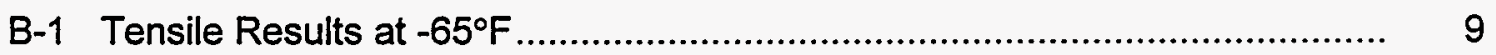

B-2 Tensile Results at Room Temperature ....................................................... 10

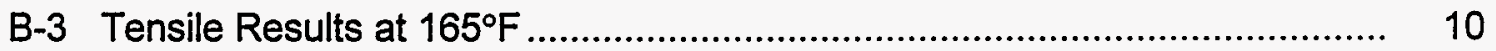

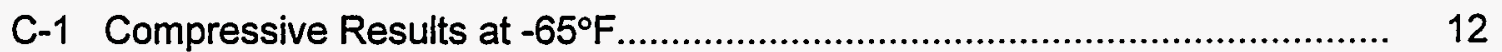

C-2 Compressive Results at Room Temperature............................................... 13

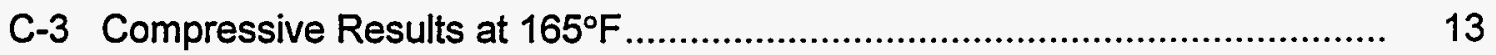

D-1 Lap Shear Results .......................................................................... 16

E-1 Coefficient of Thermal Expansion (CTE) ……........................................... 19

F-1 Glass Transition Temperature by RMS ……............................................ 32

F-2 RMS Recorded Data for A12 (1:1) ...................................................... 38

F-3 RMS Recorded Data for A12 (1:2) ......................................................... 41

F-4 RMS Recorded Data for A12 (2:3) …................................................... 44

F-5 RMS Recorded Data for EC2216 …...................................................... 47

F-6 RMS Recorded Data for EA9309 …....................................................... 50

G-1 Glass Transition Temperature by DMA …..................................................

H-1 Thermal Decomposition Temperature and Residue .................................... 63

I-1 Adhesive Mix Ratios............................................................................ 71

1-2 Heat of Reaction, Activation Energy, Frequency Factor ............................... 72

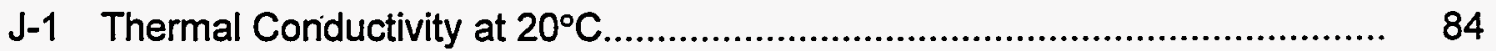

K-1 Linear Shrinkage, Volume Shrinkage, Density, and Specific 


\begin{abstract}
Tests were performed to characterize the mechanical and thermal properties of selected optical adhesives to identify the most likely candidate which could survive the operating environment of the Direct Optical Initiation (DOI) program. The DOI system consists of a high power laser and an optical module used to split the beam into a number of channels to initiate the system. The DOI requirements are for a high shock environment which current military optical systems do not operate. Five candidate adhesives were selected and evaluated using standardized test methods to determine the adhesives' physical properties. EC2216, manufactured by $3 M$, was selected as the baseline candidate adhesive based on the test results of the physical properties.
\end{abstract}

\title{
Summary
}

Tests were performed to characterize the mechanical and thermal properties of selected optical adhesives to identify the most likely candidate which could survive the operating environment of the Direct Optical Initiation (DOI) program. The DOI system consists of a high power laser and an optical module used to split the beam into a number of channels to initiate the system. The DOI system is designed for a high shock and extreme thermal environment which existing military optical packages currently do not operate. To survive the environment, the optical elements making up the system will need to be borided into mounts. Mechanical mounting could induce localized high stress areas, causing the optical element to fracture.

Five candidate adhesives were selected and evaluated using standardized test methods to determine the adhesives' physical properties. EC2216, manufactured by 3M, was selected as the baseline adhesive based on the test results of the physical properties. It had good tensile properties, compression, and lap shear strength; and the coefficient of thermal expansion matches well with Invar mounts and fused silica optics. 


\section{DISCUSSION}

\section{SCOPE AND PURPOSE}

This work was performed to characterize and identify a probable optical adhesive to be used on the Direct Optical Initiation (DOI) firing system. The DOI firing system uses a laser to generate a high energy beam which is then split into numerous channels, focused into fibers, and then used to launch optical slapper detonators. This study was to characterize adhesives based on standard test methods and use the results as a basis for selecting the most likely candidate for the DOI optical adhesive. This will also help identify tests which may be most pertinent for future quality acceptance criteria.

Three adhesives (one with three different mix ratios) were chosen as likely candidates. These adhesives were subjected to 11 different tests measuring 18 parameters. The tests encompassed both mechanical and thermal property measurements.

\section{ACTIVITY}

\section{Background}

The DOI firing system is being designed to withstand current and proposed weapon environments and, as such, must withstand thermal environments of $-55^{\circ} \mathrm{C}$ to $75^{\circ} \mathrm{C}$ and mechanical shock environments of $3000 \mathrm{~g}$ 's. The laser and other optical systems will use adhesives to bond the optical elements to ruggadized mounts to maintain the required alignments. Current military applications do not cover all the environmental conditions or have the lifetime requirements necessary for nuclear weapon applications.

Three adhesives were chosen as probable candidates based on previous use in military optical systems. These were A12, manufactured by Armstrong Products Company; EC2216 (gray), manufactured by 3M Industrial Tape and Specialties Division; and EA9309.3, manufactured by Aerospace Adhesives and Structural Materials. The A12 was tested in three different mix ratios since it had been used that way in previous military optical systems. The other two were mixed in accordance with manufacturer's recommendations.

Ten different tests were identified to characterize the adhesives. Table 1 lists the test method and the properties determined from that test and identifies an Appendix which contains information for that particular test. Upon completion of a test, a report was generated and distributed. These reports contain description of the purpose of the test, background, cure schedule, test procedure, and results along with copies of the ASTM or other standard. These reports are included as Appendices. 
Table 1. Summary of Testing Methods

\begin{tabular}{|l|l|l|}
\hline Test Method & Properties Determined & Appendix \\
\hline 1) ASTM D 651 & $\begin{array}{l}\text { Tensile Strength @ Temp } \\
\text { Modulus of Elasticity @ Temp } \\
\text { Percent Elongation @ Temp }\end{array}$ & B \\
\hline 2) ASTM D 695 & $\begin{array}{l}\text { Compressive Strength @ Temp } \\
\text { Compressive Moduli @ Temp }\end{array}$ & C \\
\hline 3) ASTM D1002 & Lap Shear on Al @ Temp & D \\
\hline $\begin{array}{c}\text { 4) Rheometric Mechanical } \\
\text { Spectrometry (RMS) }\end{array}$ & $\begin{array}{l}\text { Shear Moduli } \\
\text { Glass Transition Temp }\end{array}$ & F \\
\hline $\begin{array}{c}\text { 5) Dynamic Mechanical } \\
\text { Analysis (DMA) }\end{array}$ & $\begin{array}{l}\text { Tensile Moduli } \\
\text { Glass Transition Temp }\end{array}$ & G \\
\hline $\begin{array}{c}\text { 6) Thermogravimetric } \\
\text { Analysis (TGA) }\end{array}$ & $\begin{array}{l}\text { Thermal Stability } \\
\text { Weight Loss }\end{array}$ & H \\
\hline $\begin{array}{c}\text { 7) Differential Scanning } \\
\text { Calorimeter (DSC) }\end{array}$ & $\begin{array}{l}\text { Kinetics of Cure } \\
\text { Specific Heat Vs Temp }\end{array}$ & I \\
\hline 8nalysis (TMA) & Coefficient of Thermal Expansion & E \\
\hline 9) ASTM E 1225 & Thermal Conductivity & $\mathrm{J}$ \\
\hline 10) ASTM D 792 & Specific Gravity & $\mathrm{K}$ \\
\hline
\end{tabular}

Once the testing was completed, the data was analyzed to determine the optimal adhesive which would provide the best properties for the thermal and mechanical environments identified for DOI.

Additional testing to try to determine Poisson ratio at room temperature was later performed on the EC2216 to generate data for finite analysis of the mounted optics.

\section{Results}

After review of the data, it was determined that EC2216 had the best properties to survive the environments for DOI without affecting the optical system. Table 2 shows a summary of the results of the testing for the EC2216. 
Table 2. Properties of EC2216

$\begin{array}{lccc}\text { Property } & \underline{\underline{-65^{\circ} \mathrm{F}}} & \text { Room Temperature } & \underline{165^{\circ} \mathrm{F}} \\ \text { Tensile Strength (psi) } & 7,340 & 2,690 & 610 \\ \text { Tensile Moduli (psi) } & 838,000 & 10,100 & 5,140 \\ \text { Elongation (\%) } & 1.04 & 35 & 11.9 \\ \text { Comp. Prop. Limit (psi) } & 9,450 & 286 & 397 \\ \text { Ult. Comp. Strain (psi) } & 16,900 & 1,960 & 625 \\ \text { Ult. Comp. Stress (\%) } & 4.61 & 10 & 10 \\ \text { Comp. Moduli (psi) } & 905,000 & 53,900 & 6,060 \\ \text { Lap Shear (psi) } & 2,480 & 3,520 & 865\end{array}$

Poisson Ratio (room temperature): 0.592

Coefficient of Thermal Expansion: Below Tg: $39.4 \times 10^{-6} \mathrm{in} / \mathrm{in}^{\circ} \mathrm{F}$

$$
\left(\mathrm{Tg}=84.9^{\circ} \mathrm{F}\right) \quad \text { Above } \mathrm{Tg}: 105.6 \times 10^{-6} \mathrm{in} / \mathrm{in}^{\circ} \mathrm{F}
$$

RMS Glass Transition Temperature: $102.2^{\circ} \mathrm{F}$

DMA Glass Transition Temperature: $69.4^{\circ} \mathrm{F}$

Thermal Decomposition Temperature: $651.9^{\circ} \mathrm{F}$

Residue (percent filler) 30.3

Heat of Reaction: $164.6 \mathrm{~J} / \mathrm{gm}$

Activation Energy: $51.1 \mathrm{Kj} / \mathrm{mole}$

Thermal conductivity (at $20^{\circ} \mathrm{C}$ ): $0.2468 \mathrm{BTU} / \mathrm{hr}-\mathrm{ft}-{ }^{\circ} \mathrm{F}$

Linear Shrinkage: $1.40 \%$

Volume Shrinkage: $4.06 \%$

Density: $0.0493 \mathrm{lb} / \mathrm{in}^{3}$

Specific Gravity: 1.366

\section{ACCOMPLISHMENTS}

Five candidate optical adhesives were tested in accordance with standard test methods to determine which adhesive had the properties required to hold mounted optics to survive DOI environments. These tests concluded that $\mathrm{EC} 2216$, manufactured by $3 \mathrm{M}$, is the leading choice for the DOI application. It has good strength and expansion properties through the temperature extremes. The properties of EC2216 will enable properly designed bonded, mounted optics to survive $3000-\mathrm{g}$ shock environments and temperature extremes of $-55^{\circ} \mathrm{C}$ to $75^{\circ} \mathrm{C}$. Test method to ensure compliant material could be the lap shear test since it is not dependent upon forming 
parts made of the adhesive and then testing. The lap shear test looks at both the bonding to a substrate and the properties of the material.

\section{FUTURE WORK}

The results of these tests indicate that optical systems can be designed to withstand weapon environments using EC2216. The effects of a thirty-year shelf life are unknown. Existing military systems using adhesives to bond optics do not have the long life requirements, and, thus, there is no data to the effect of long-term aging. The next phase will be an accelerated aging study over two years. Both $\mathrm{EC} 2216$ and the 2:3 mix ratio $\mathrm{A} 12$ will be tested due to the length of the aging study. This will provide a backup adhesive if the EC2216 fails to perform adequately in the other tests. 
Appendix A

Mix Ratio and Cure Schedules 


\section{Mix Ratio and Cure Schedule}

The optical adhesive test samples were mixed and cured per Table A-1 for all tests. The Kinetics of Cure test samples were cured using different schedules. The cure schedule for those tests are defined in Appendix I,

Table A-1. Mix Ratio and Cure Schedule

\begin{tabular}{|c|c|c|c|}
\hline \multirow[t]{2}{*}{ Adhesive } & \multicolumn{2}{|c|}{ Mix Ratio* } & \multirow[t]{2}{*}{ Cure Schedule } \\
\hline & A Comp. & B Comp. & \\
\hline A12 1:1 & 1 & 1 & 24 hrs @ room temp., 2 hrs @ 160 \\
\hline A12 1:2 & 1 & 2 & 24 hrs @ room temp., 2 hrs @ $160^{\circ} \mathrm{F}$ \\
\hline A12 2:3 & 2 & 3 & 24 hrs @ room temp., 2 hrs @ 160F \\
\hline EC2216 (Gray) & 1.4 & 1 & 16 hrs @ room temp., 16 hrs @ $160^{\circ} \mathrm{F}$ \\
\hline EA9309.3NA & 10 & 2.2 & 7 days at room temperature \\
\hline
\end{tabular}

* Material was mixed based on weight, i.e., 10:2.2 would be mixed using 10 grams of A component mixed with 2.2 grams of B component or some factor thereof. 
Appendix B

\section{Tensile Strength}




\section{Tensile Strength}

\section{Test Purpose:}

The purpose of this test is to determine the tensile strength, modulus of elasticity, and percent elongation over temperature of the optical adhesives .

\section{Background:}

The testing is per ASTM D 651 and is included in Appendix L.

\section{Sample Preparation:}

Fifteen samples of each material were mixed and cured per Table A-1 in Appendix A. After curing, the sample's length and width were measured and then the top and bottom surfaces were machined to 0.125 inch final thickness.

\section{Test Procedure:}

Testing is per ASTM D 651 and is included in Appendix L.

\section{Test Results:}

The following tables lists the average and standard deviation (s) for the tensile strength, tensile moduli, and percent elongation. Table $\mathrm{B}-1$ shows the results at $-65^{\circ} \mathrm{F}$, Table B-2 is at room temperature, and Table B-3 is at $165^{\circ} \mathrm{F}$. Five samples of each material were tested at each temperature.

Table B-1: Tensile Results at $-65^{\circ} \mathrm{F}$

\begin{tabular}{|l|c|c|c|c|c|c||}
\hline \multicolumn{1}{|c|}{ Material } & \multicolumn{2}{c|}{ Tensile Strength } & \multicolumn{2}{c|}{ Tensile Moduli } & \multicolumn{2}{c|}{ Elongation } \\
\hline \hline & (psi) & s & (psi) & s & (\%) & s \\
\hline \hline A12 1:1 & 8,540 & 1280 & 549,000 & 50,900 & 1.56 & 0.50 \\
\hline A12 2:3 & 8,490 & 2190 & 576,000 & 69,000 & 1.68 & 0.73 \\
\hline A12 1:2 & 7,840 & 1870 & 500,000 & 80,000 & 2.00 & 0.69 \\
\hline EC 2216 (gray) & 7,370 & 1090 & 838,000 & 119,000 & 1.04 & 0.18 \\
\hline EA 9309.3 NA & 7,410 & 1010 & 390,000 & 51,500 & 2.17 & 0.39 \\
\hline
\end{tabular}


Table B-2: Tensile Results at Room Temperature

\begin{tabular}{||l|c|c|c|c|c|c||}
\hline \multicolumn{1}{|c|}{ Material } & \multicolumn{2}{c|}{ Tensile Strength } & \multicolumn{2}{c|}{ Tensile Moduli } & \multicolumn{2}{c||}{ Elongation } \\
\hline \hline & (psi) & s & (psi) & s & (\%) & s \\
\hline \hline A12 1:1 & 6,390 & 437 & 411,000 & 52,000 & 2.57 & 0.72 \\
\hline A12 2:3 & 3,250 & 419 & 191,000 & 31,000 & 13.1 & 7.3 \\
\hline A12 1:2 & 1,190 & 85 & 2,390 & 344 & 87 & 7.6 \\
\hline EC 2216 (gray) & 2,690 & 356 & 10,100 & 3,630 & 35 & 5.0 \\
\hline EA 9309.3 NA & 5,620 & 167 & 363,000 & 150,000 & 2.8 & 0.67 \\
\hline \hline
\end{tabular}

Table B-3: Tensile Results at $165^{\circ} \mathrm{F}$

\begin{tabular}{||l|c|c|c|c|c|c||}
\hline \hline \multicolumn{1}{|c|}{ Material } & \multicolumn{2}{|c|}{ Tensile Strength } & \multicolumn{2}{c|}{ Tensile Moduli } & \multicolumn{2}{c|}{ Elongation } \\
\hline \hline & (psi) & $\mathbf{s}$ & (psi) & s & $(\%)$ & s \\
\hline \hline A12 1:1 & 893 & 134 & 7,320 & 1,980 & 16.0 & 2.0 \\
\hline A12 2:3 & 263 & 29 & 1,500 & 23 & 17.8 & 2.1 \\
\hline A12 1:2 & 135 & 25 & 844 & 74 & 16.7 & 3.6 \\
\hline EC 2216 (gray) & 610 & 66 & 5,140 & 112 & 11.9 & 1.1 \\
\hline EA 9309.3 NA & 1,280 & 184 & 89,500 & 46,600 & 21.7 & 4.7 \\
\hline
\end{tabular}


Appendix C

Compressive Strength 


\section{Compressive Strength}

\section{Test Purpose:}

The purpose of this test is to determine the compressive strength and compressive moduli over temperature of the optical adhesives.

\section{Background:}

The testing is per ASTM D 695 and is included in Appendix L.

\section{Sample Preparation:}

Fifteen samples, $0.5 \hat{i} \times 0.5 \hat{1} \times 1.0^{\prime \prime}$ long, were mixed and cured per Table A-1 in Appendix A. After curing, the samples were machined flat.

\section{Test Procedure:}

The test procedure was per ASTM D 695 and is included in Appendix L.

\section{Test Results:}

The following tables list the average and standard deviation (s) for the proportional limit, ultimate compressive stress and strain, and the compressive moduli. Table $\mathrm{C}-1$ shows the results at $-65^{\circ} \mathrm{F}$, Table C-2 is at room temperature, and Table C-3 is at $165^{\circ} \mathrm{F}$.

Table C-1: Compressive Results at $-65^{\circ} \mathrm{F}$

\begin{tabular}{|c|c|c|c|c|c|c|c|c|}
\hline \multirow[t]{2}{*}{ Material } & \multicolumn{2}{|c|}{$\begin{array}{c}\begin{array}{c}\text { Proportional } \\
\text { Limit }\end{array} \\
\end{array}$} & \multicolumn{4}{|c|}{$\begin{array}{c}\text { Ultimate } \\
\text { Compressive }\end{array}$} & \multicolumn{2}{|c|}{$\begin{array}{c}\text { Compressive } \\
\text { Moduli }\end{array}$} \\
\hline & (psi) & $\mathbf{s}$ & $\begin{array}{l}\text { Stre } \\
\text { (psi) }\end{array}$ & $\mathbf{s}$ & $\begin{array}{l}\text { Str } \\
(\%)\end{array}$ & in & (psi) & $\mathbf{s}$ \\
\hline A12 $1: 1$ & 11,040 & 783 & 19,100 & 352 & 6.17 & .21 & 673,000 & 18,400 \\
\hline A12 2:3 & 9,600 & 196 & 16,600 & 195 & 5.60 & .32 & 616,000 & 6,630 \\
\hline A12 1:2 & 8,000 & 450 & 13,700 & 311 & 5.24 & .26 & 520,000 & 15,700 \\
\hline EC 2216 (gray) & 9,450 & 286 & 16,900 & 207 & $\overline{4.61}$ & .35 & 905,000 & 16,700 \\
\hline EA 9309.3 NA & 7,540 & 309 & 16,900 & 523 & 8.92 & .64 & 439,000 & 11,900 \\
\hline
\end{tabular}


Table C-2: Compressive Results at Room Temperature

\begin{tabular}{|c|c|c|c|c|c|c|c|c|}
\hline \multirow[t]{2}{*}{ Material } & \multicolumn{2}{|c|}{$\begin{array}{c}\text { Proportional } \\
\text { Limit }\end{array}$} & \multicolumn{4}{|c|}{$\begin{array}{c}\text { Ultimate } \\
\text { Compressive }\end{array}$} & \multicolumn{2}{|c|}{$\begin{array}{c}\text { Compressive } \\
\text { Moduli }\end{array}$} \\
\hline & (psi) & $\mathbf{s}$ & $\begin{array}{c}\text { Strc } \\
\text { (psi) }\end{array}$ & $s$ & $\begin{array}{r}\text { St } \\
(\%)\end{array}$ & ${ }^{i n}$ & (psi) & $\mathbf{s}$ \\
\hline A12 1:1 & 5,150 & 106 & 9,140 & 221 & 3.63 & .01 & 446,000 & 16,200 \\
\hline A12 2:3 & 2,740 & 200 & 4,120 & 197 & 4.36 & .22 & 180,000 & 15,900 \\
\hline A12 1:2 & 236 & 50 & 461 & 25 & 10.0 & & 10,500 & 999 \\
\hline EC 2216 (gray) & 286 & 26 & 1,960 & 65 & 10.0 & & 53,900 & 8510 \\
\hline EA 9309.3 NA & 4,650 & 383 & 7,710 & 153 & 4.24 & .24 & 338,000 & 6460 \\
\hline
\end{tabular}

Table C-3: Compressive Results at $165^{\circ} \mathrm{F}$.

\begin{tabular}{|c|c|c|c|c|c|c|c|c|}
\hline \multirow[t]{2}{*}{ Material } & \multicolumn{2}{|c|}{$\begin{array}{c}\text { Proportional } \\
\text { Limit }\end{array}$} & \multicolumn{4}{|c|}{$\begin{array}{c}\text { Ultimate } \\
\text { Compressive }\end{array}$} & \multicolumn{2}{|c|}{$\begin{array}{c}\text { Compressive } \\
\text { Moduli }\end{array}$} \\
\hline & (psi) & $s$ & $\begin{array}{c}\text { Str } \\
\text { (psi) }\end{array}$ & s & $\begin{array}{l}\text { Str } \\
\text { (\%) }\end{array}$ & in & (psi) & $s$ \\
\hline A12 $1: 1$ & 470 & 44 & 751 & 16 & 10.0 & & 7,220 & 161 \\
\hline A12 2:3 & 112 & 7.3 & 183 & 4.1 & 10.0 & & 1,770 & 46.6 \\
\hline A12 $1: 2$ & 54 & $\overline{3.5}$ & 97.3 & .80 & 10.0 & & 948 & 11.7 \\
\hline EC 2216 (gray) & 397 & 12 & 625 & 13 & 10.0 & & 6,060 & 138 \\
\hline EA 9309.3 NA & 390 & 59 & 1,230 & 67 & 10.0 & & 44,800 & $\overline{7270}$ \\
\hline
\end{tabular}




\section{Appendix D \\ Lap Shear}




\section{Lap Shear}

\section{Test Purpose:}

The purpose of this test is to determine the lap shear strength of the optical adhesives on aluminum test samples .

\section{Background:}

The testing is per ASTM D 1002 and is included in Appendix L.

\section{Sample Preparation:}

Fifteen samples of each adhesive were mixed, applied, and cured per Table A-1 in Appendix A.

The surfaces of the aluminum plates were etched per 9916023 (included in Appendix L) prior to the application of the adhesive. This process is as follows:

a) Vapor degrease in 1,1,1-Trichloroethylene per 9906006 (included in Appendix L)

b) Dry

c) Etch (in Sodium dichromate, sulfuric acid and water solution at $150^{\circ} \mathrm{F}$ for 10 minutes per 9906010 - included in Appendix L)

d) Rinse in deionized (DI) water

e) Dry

\section{Test Procedure:}

Five samples of each adhesive were prepared and tested in accordance with ASTM D 1002 and are included in Appendix L.

\section{Test Results:}

Table D-1 shows the average lap shear strength and the standard deviation (s) for $-65^{\circ} \mathrm{F}$, room temperature, and $165^{\circ} \mathrm{F}$. 
Table D-1: Lap Shear Results

\begin{tabular}{|c|c|c|c|c|c|c|}
\hline \multirow{3}{*}{ Material } & \multicolumn{6}{|c|}{ Lap Shear Strength } \\
\hline & \multicolumn{2}{|c|}{$-65^{\circ} \mathrm{F}\left(-54^{\circ} \mathrm{C}\right)$} & \multicolumn{2}{|c|}{ R.T. } & \multicolumn{2}{|c|}{$165^{\circ} \mathrm{F}\left(74^{\circ} \mathrm{C}\right)$} \\
\hline & (psi) & $\mathbf{s}$ & (psi) & $\mathbf{s}$ & (psi) & $\mathbf{s}$ \\
\hline A12 1:1 & 2,710 & 310 & 3,460 & 382 & 1,400 & 72.2 \\
\hline A12 2:3 & 3,760 & 449 & 3,140 & 82.5 & 334 & 9.5 \\
\hline A12 1:2 & 2,880 & 389 & 1,720 & 22.8 & 220 & 9.1 \\
\hline EC 2216 (gray) & 2,480 & 305 & 3,520 & 83.7 & 865 & 233 \\
\hline EA 9309.3 NA & 6,390 & 795 & 4,530 & 192 & 2,100 & 10.1 \\
\hline
\end{tabular}




\section{Appendix E \\ Coefficient of Thermal Expansion}




\section{Coefficient of Thermal Expansion}

\section{Test Purpose:}

This test will determine the glass transition temperature $(\mathrm{Tg})$ and coefficient of thermal expansion (CTE) above and below the $\mathrm{Tg}$.

\section{Background:}

Thermal mechanical analysis (TMA) is a technique in which deformation of a substance is measured under a static load or a function of temperature as in this case. TMA is used to determine glass transition temperature $(\mathrm{Tg})$ and coefficient of thermal expansion (CTE) above and below the $\mathrm{Tg}$.

The setup uses a DuPont 9900 controller with a 943 TMA which uses a linear variable differential transformer (LVDT) to detect changes in sample dimensions during heating. The quartz probe, which contains the core of the LVDT, is placed in contact with the sample. As probe displacement occurs, due to changes in the sample dimension, a movement of the LVDT core results in a linear voltage output proportional to probe displacement.

\section{Sample Preparation:}

One sample approximately $0.250^{\prime \prime}$ diameter $\times 0.3^{\prime \prime}$ long was made and tested from each adhesive. The samples were mixed and cured per Table A-1 in Appendix A.

\section{Test Procedure:}

The samples were tested with a TA instrument TMA using an expansion probe with 1 gram tray weight in air from $-60^{\circ} \mathrm{C}$ to $80^{\circ} \mathrm{C}$ at $5^{\circ} \mathrm{C}$ per minute then back to room temperature and up to $80^{\circ} \mathrm{C}$ at $5^{\circ} \mathrm{C}$ per minute.

\section{Test Results:}

Figures E-1 thru E-5 show the dimensional change as a function of temperature. The coefficient of thermal expansion (representative by a) is determined by the slope of the curve. There are two distinct slopes and intersection of the two lines gives the glass transition temperature (a more accurate way to determine glass transition temperature is Rheometric Mechanical Spectrometry and is discussed in Appendix F). Table E-1 shows the glass transition temperature $(\mathrm{Tg})$ and the coefficient of thermal expansion (CTE) below and above the $\mathrm{Tg}$. 
Figures E-6 thru E-10 are also plots of the dimensional change as a function of temperature. In this case the material is allowed to cool to room temperature and then reheated. As can be seen from the curves, there is a definite shift between the heating and cooling phases although the slope of the curves remain similar. There is a fair amount of stress relaxation in the EA9309.3 NA material as evident in Figure E-10.

Table E-1: Coefficient of Thermal Expansion (CTE)

\begin{tabular}{|c|c|c|c|c|c|c|}
\hline \multirow[t]{2}{*}{ Material } & \multicolumn{2}{|c|}{$\begin{array}{c}\text { Glass Transition } \\
\text { Temperature }\end{array}$} & \multicolumn{2}{|c|}{$\begin{array}{c}\text { CTE } \\
\text { below } \mathrm{T}_{\mathbf{g}}\end{array}$} & \multicolumn{2}{|c|}{$\begin{array}{c}\text { CTE } \\
\text { above } T_{g}\end{array}$} \\
\hline & $\left.{ }^{\circ} \mathrm{F}\right)$ & $\left({ }^{\circ} \mathrm{C}\right)$ & A & B & $\mathbf{A}$ & $\mathrm{B}$ \\
\hline A12 1:1 & 119.7 & 48.7 & 37.8 & 68 & 86.1 & 155 \\
\hline A12 1:2 & 104.4 & 40.2 & 50.0 & 90 & 109.4 & 197 \\
\hline A12 2:3 & 111.4 & 44.1 & 43.9 & 79 & $\overline{120.6}$ & 217 \\
\hline EC 2216 (gray) & 84.9 & 29.4 & 39.4 & 71 & $\overline{105.6}$ & 190 \\
\hline EA 9309.3 NA & 102.2 & 39.0 & 45.6 & 82 & 74.4 & 134 \\
\hline
\end{tabular}

A units: in $/$ in $/{ }^{\circ} \mathrm{F} \times 10^{-6}$

$B$ units: $\mu \mathrm{m} / \mathrm{m} /{ }^{\circ} \mathrm{C}$ 


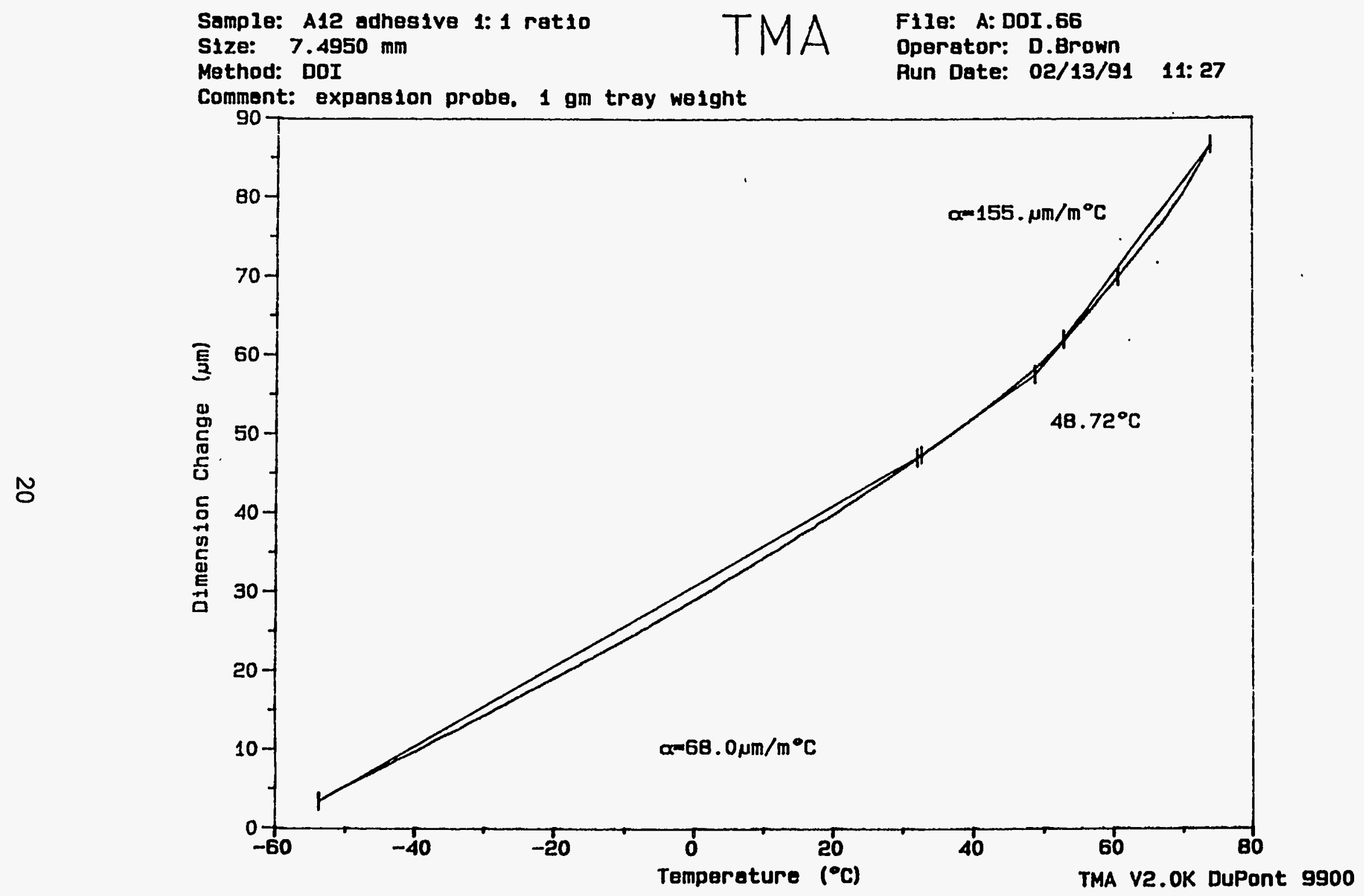

Figure E-1. Dimensional Change as a Function of Temperature for A12 1:1 Ratio 


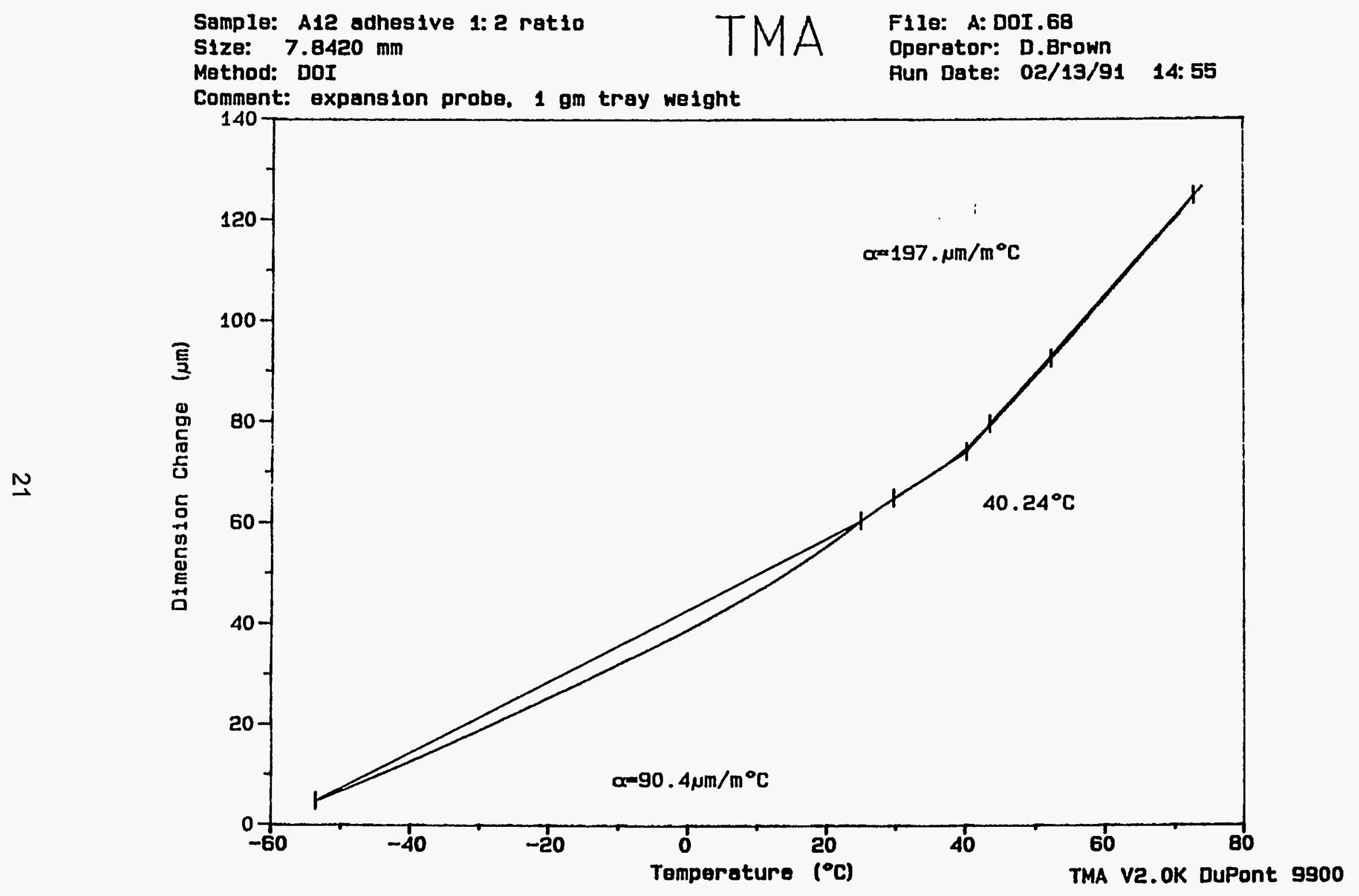

Figure E-2. Dimensional Change as a Function of Temperature for A12 1:2 Ratio 


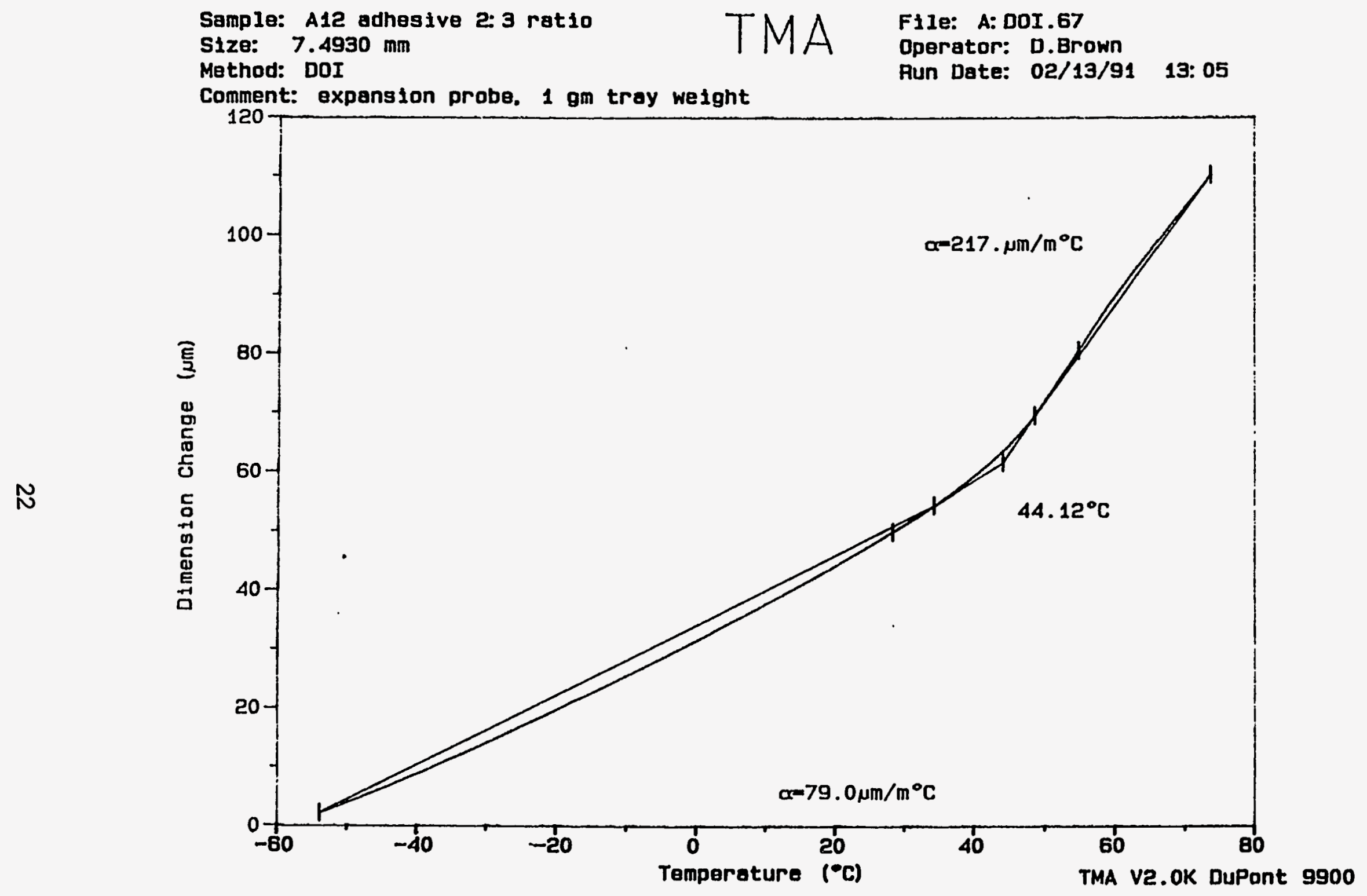

Figure E-3. Dimensional Change as a Function of Temperature for A12 2:3 Ratio 


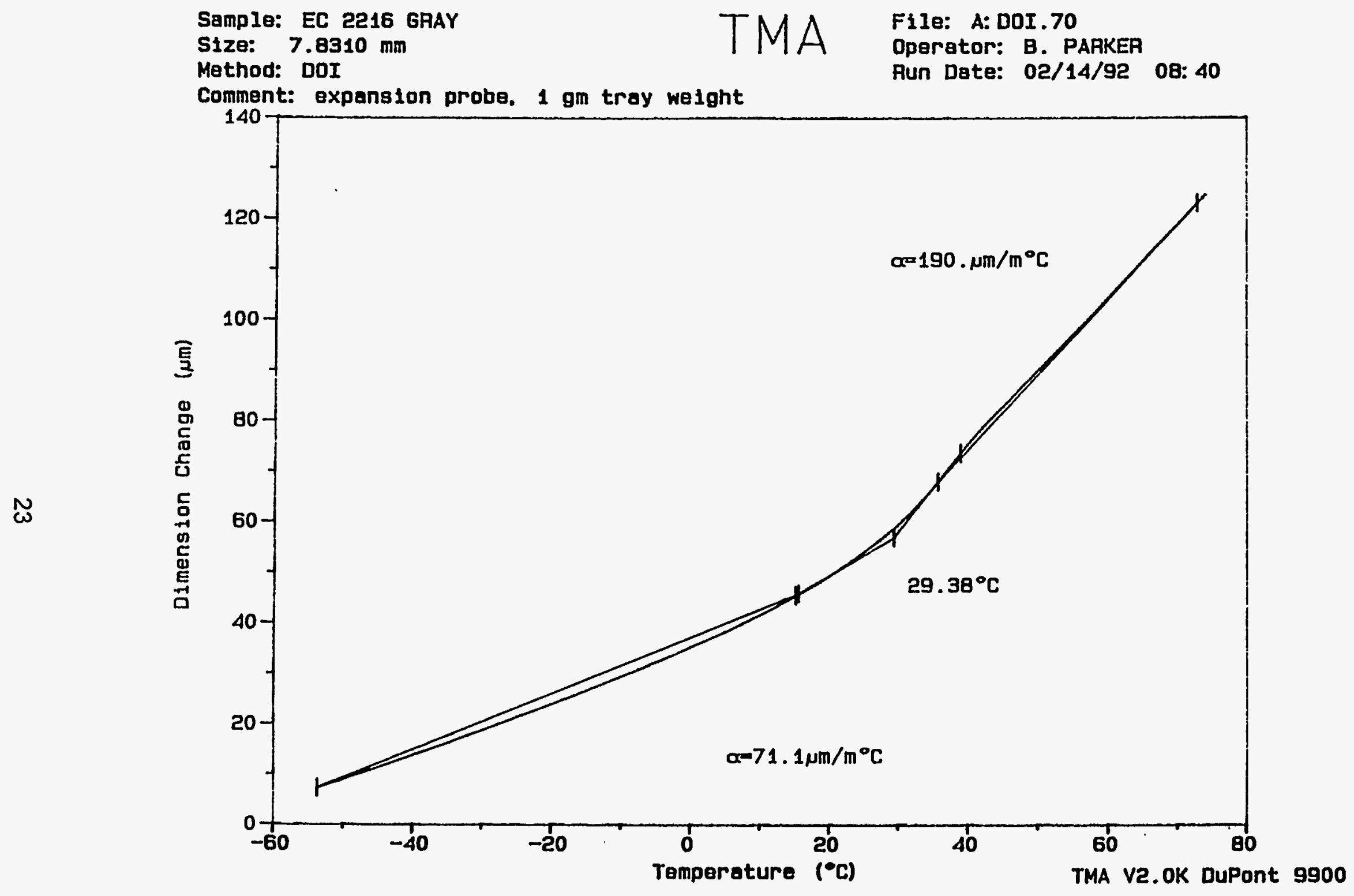

Figure E-4. Dimensional Change as a Function of Temperature for EC2216 


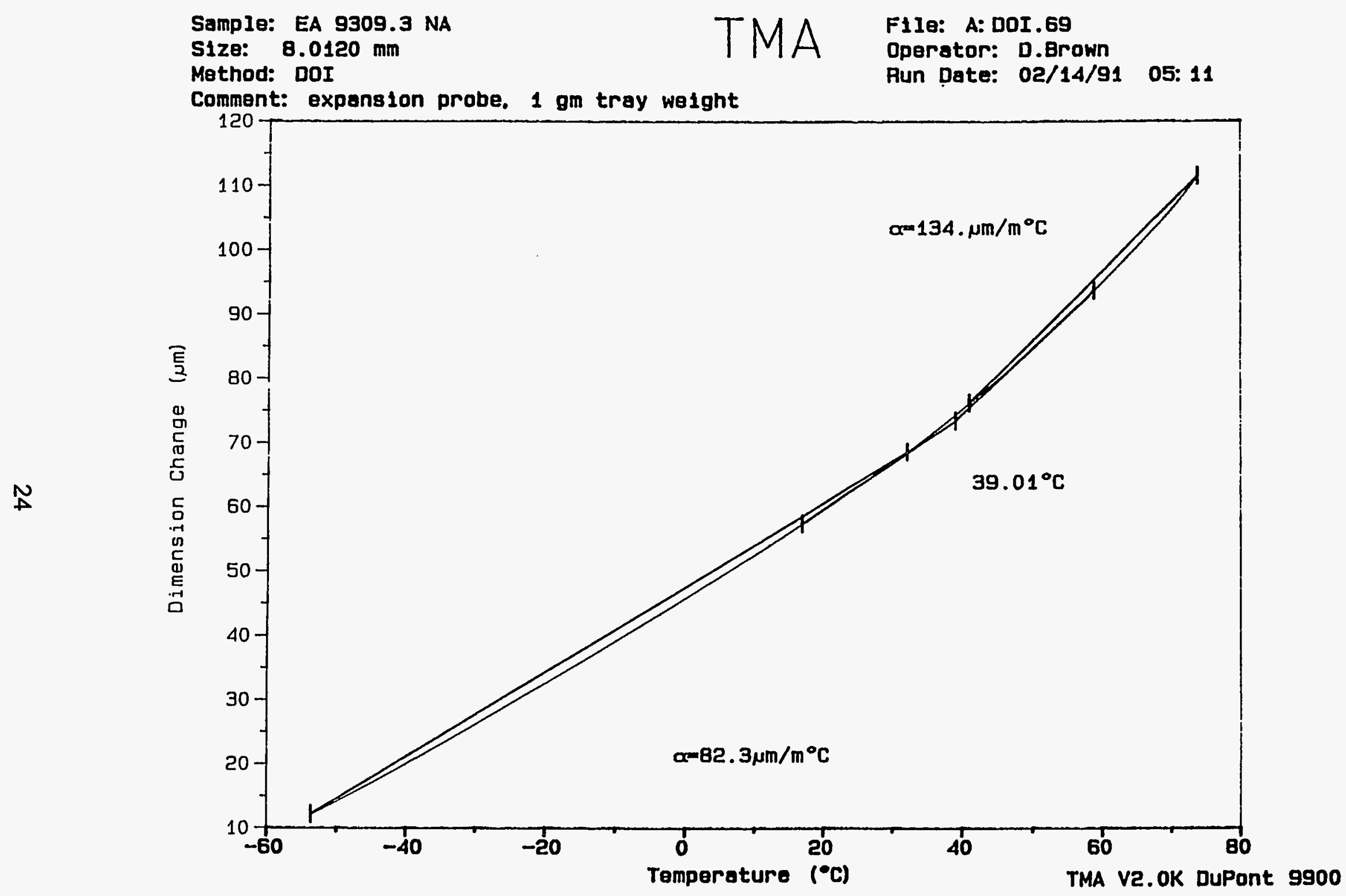

Figure E-5. Dimensional Change as a Function of Temperature for EA9309.3 NA 


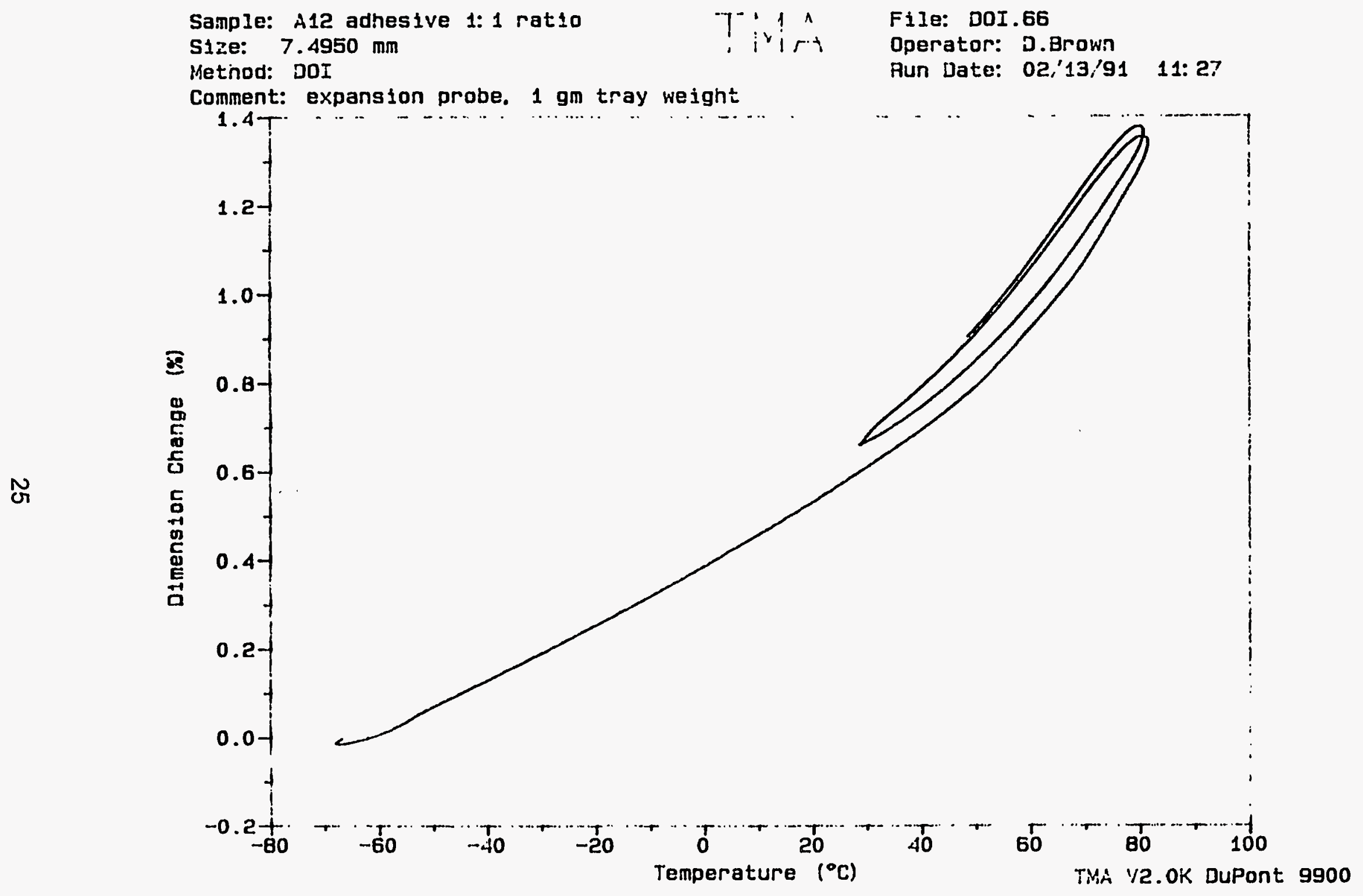

Figure E-6. Dimensional Change During Temperature Cycle for A12 1:1 Ratio 


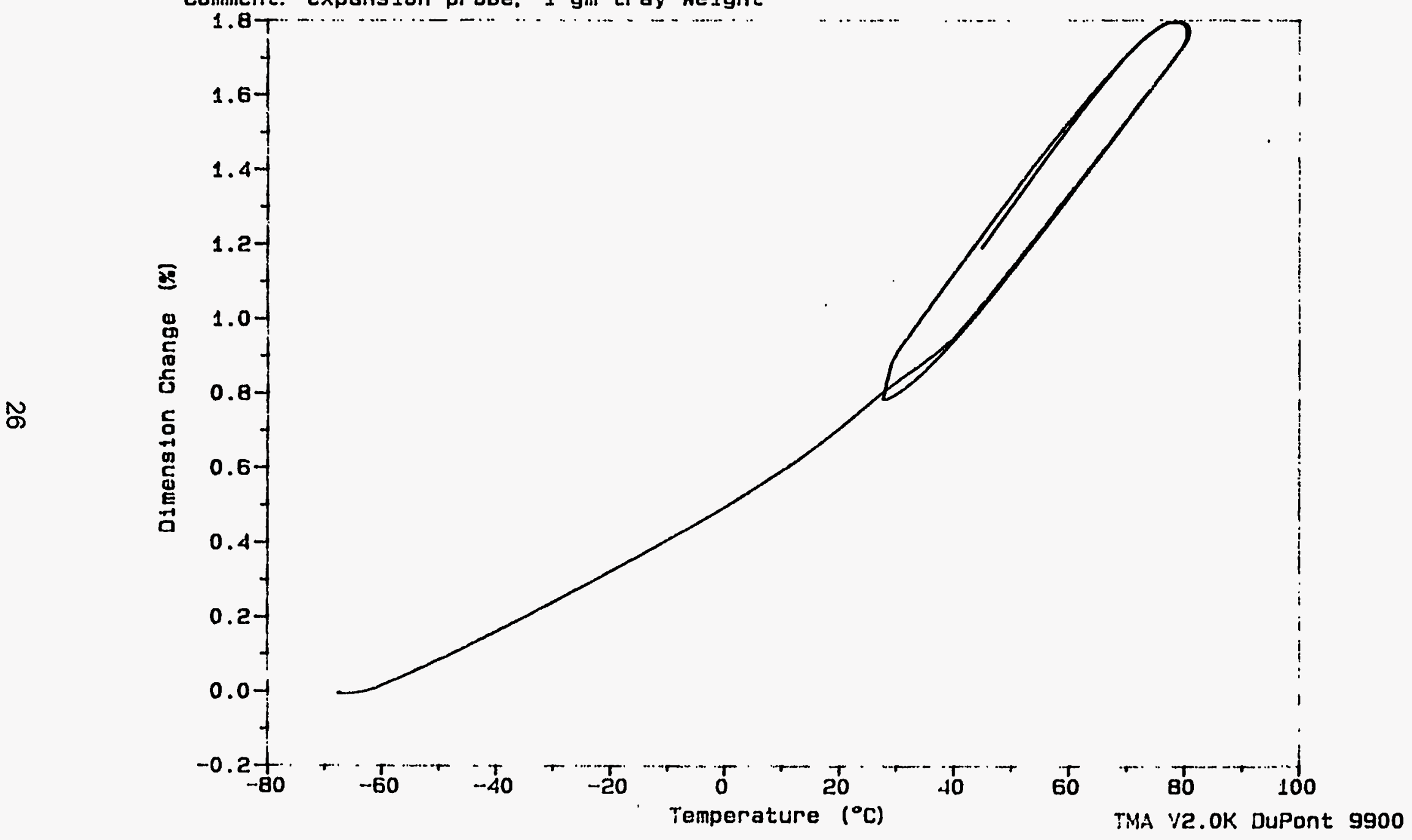

Sample: $A 12$ adhesive $1: 2$ ratio

Stze: $7.8420 \mathrm{~mm}$

Method: DOI

Comment: expansion probe, 1 gm tray weight

Operator: 0.Brown

Bun Date: 02/13/91 14:55

\%

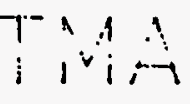

F11e: $00 \mp .68$

Figure E-7. Dimensional Change During Temperature Cycle for A12 1:2 Ratio 


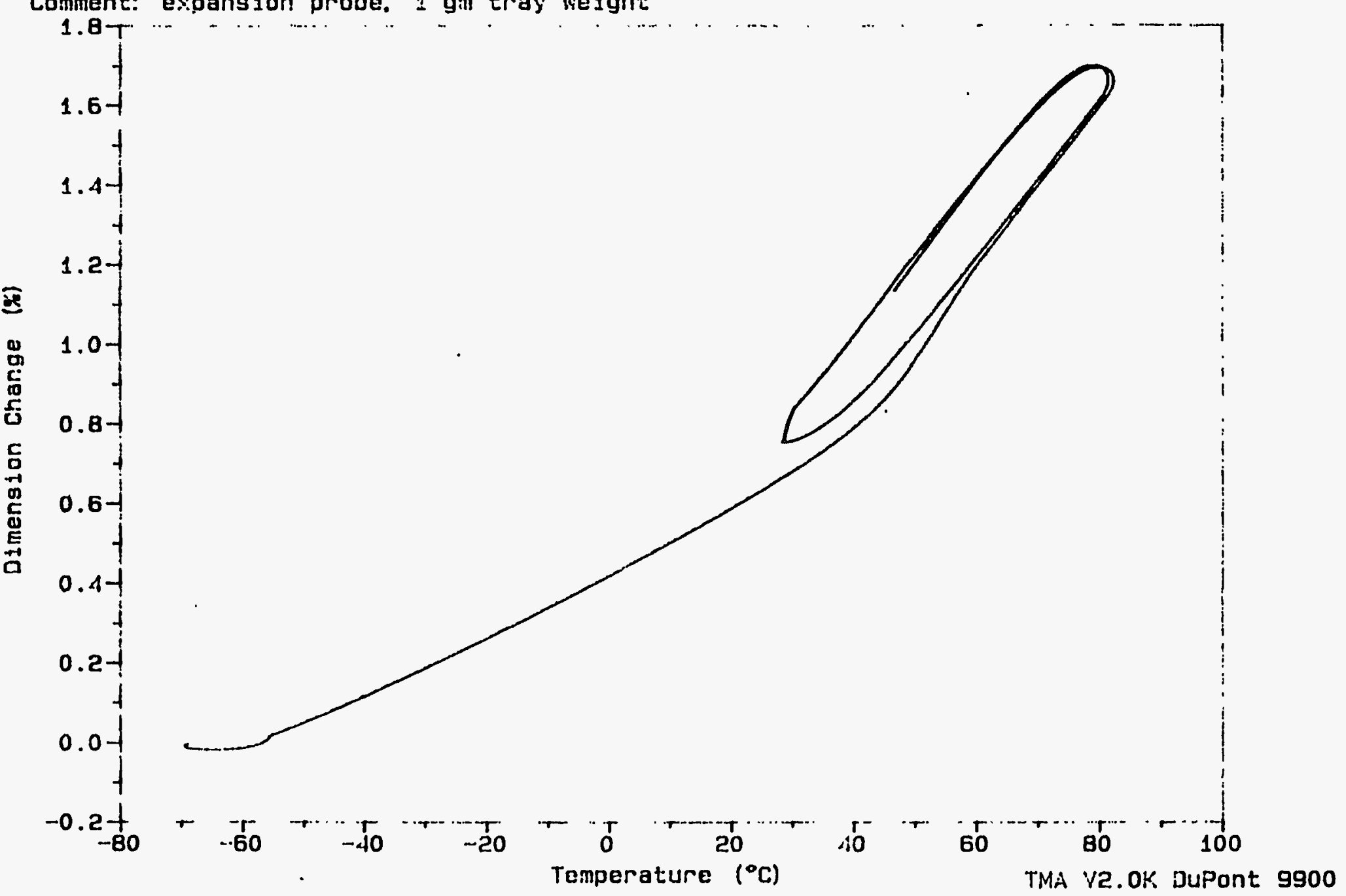

File: DOI.67

Operator: D.Brown

Aun Date: 02,13/91 13: 05
Sample: A12 adhesive 2: 3 ratio S1ze: $7.4930 \mathrm{~mm}$

Method: DOI

Comment: expansion probe. 1 gm tray biejght

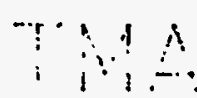

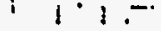

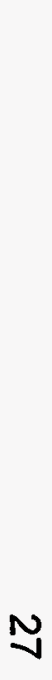

S

Figure E-8. Dimensional Change During Temperature Cycle for A12 2:3 Ratio 


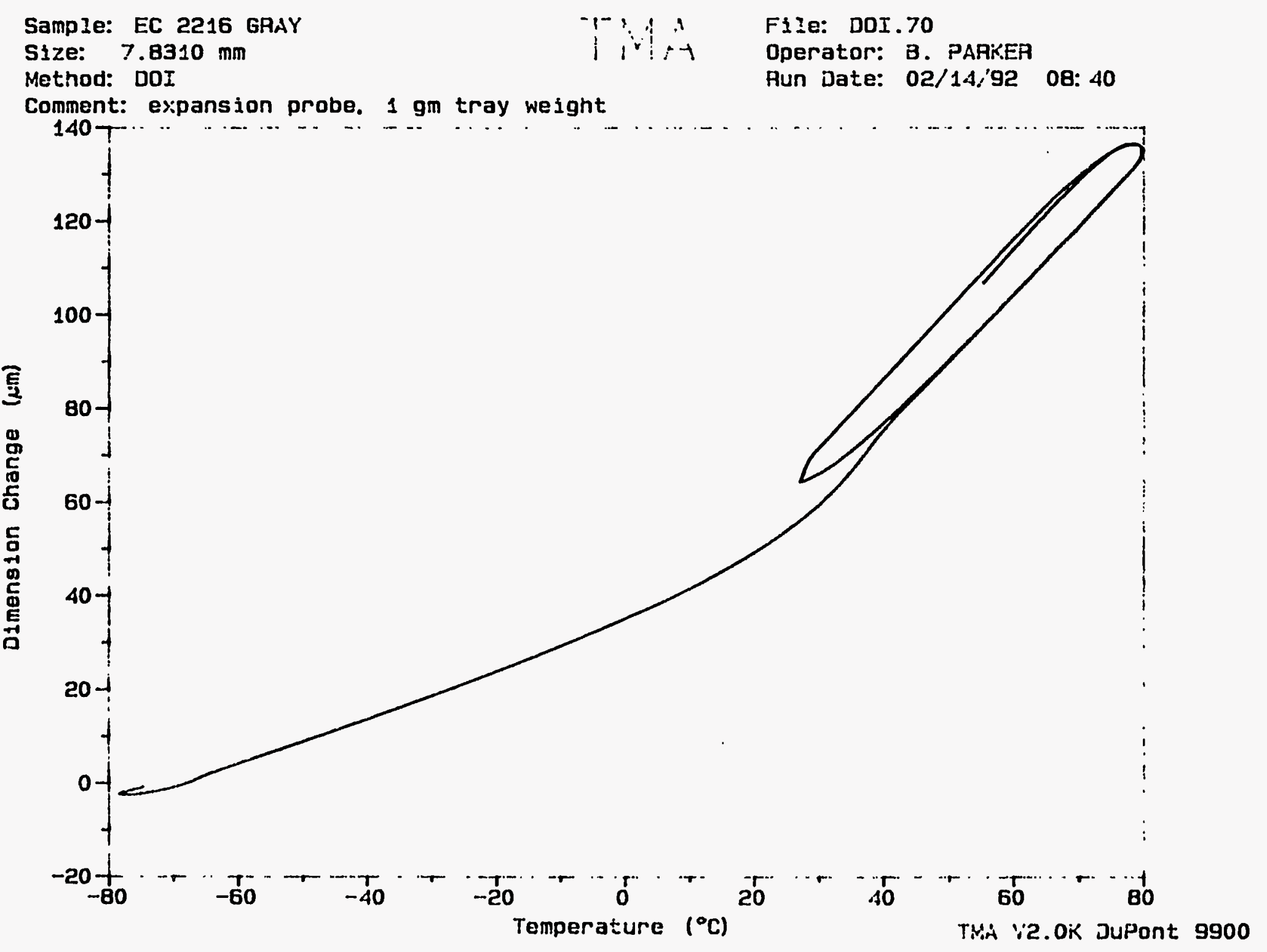

Figure E-9. Dimensional Change During Temperature Cycle for EC2216 


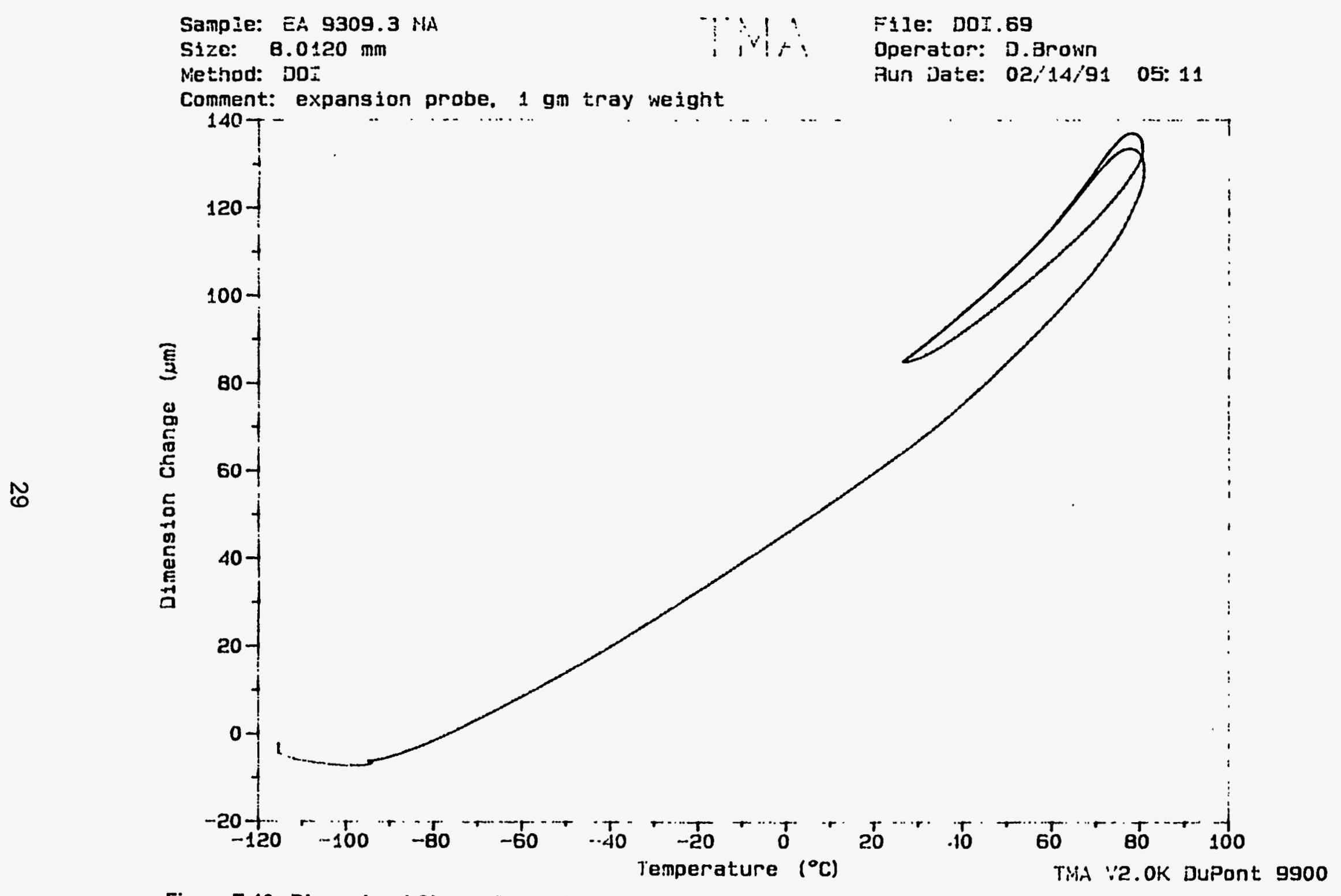

Figure E-10. Dimensional Change During Temperature Cycle for EA9309.3 NA 


\section{Appendix F \\ RMS Glass Transition Temperature}




\section{RMS Glass Transition Temperature}

\section{Test Purpose:}

The purpose of this test is to determine the shear modulus and glass transition temperature of the optical adhesives using Rheometric Mechanical Spectrometry.

\section{Background:}

Rheometric analysis is used to determine the shear modulus, damping coefficient (tan delta), and the glass transition temperature. The RMS was used in a forced torsional mode. In this mode, a rectangular sample is clamped between two grips. One grip is held fixed, and the other grip subjects the sample to a torsional oscillation of fixed amplitude. The amount of energy necessary to oscillate the sample in this manner is monitored as a function of temperature, as is the restoring force supplied by the sample. These energy measurements are converted to modulus and damping data.

\section{Sample Preparation:}

Samples, approximately $0.5^{\prime \prime} \times 0.125^{\prime \prime} \times 1.5^{\prime \prime}$, were mixed and cured per Table A-1 in Appendix A.

\section{Test Procedure:}

Rheometrics mechanical spectrometer testing was performed at $3^{\circ} \mathrm{C}$ per minute from $-65^{\circ} \mathrm{C}$ to $125^{\circ} \mathrm{C}$ in the dynamic mode. All samples were run at a frequency of 1 hertz. The strain was automatically adjusted from a minimum of $0.05 \%$ to a maximum of $2 \%$, and a measurement was recorded every minute.

\section{Test Results:}

Table F-1 shows the glass transition temperatures which are the temperatures at maximum tan delta. The curves showing the Shear Modulus ( $\left.G^{\prime}\right), G^{\prime \prime}$ and Tan Delta as a function of temperature are shown in Figures F-1 through F-5. Tables F-2 through F-6 show the recorded data at each interval. 
Table F-1: Glass Transition Temperature by RMS:

\begin{tabular}{||l|c|c||}
\hline Material & \multicolumn{2}{|c|}{$\begin{array}{c}\text { Glass Transition Temperature } \\
\left({ }^{\circ} \mathrm{C}\right)\end{array}$} \\
\hline \hline A12 1:1 & 75 & 167.0 \\
\hline A12 2:3 & 48 & 118.4 \\
\hline A12 1:2 & 40 & 104.0 \\
\hline EC 2216 (gray) & 39 & 102.2 \\
\hline EA 9309.3 NA & 87 & 188.6 \\
\hline
\end{tabular}




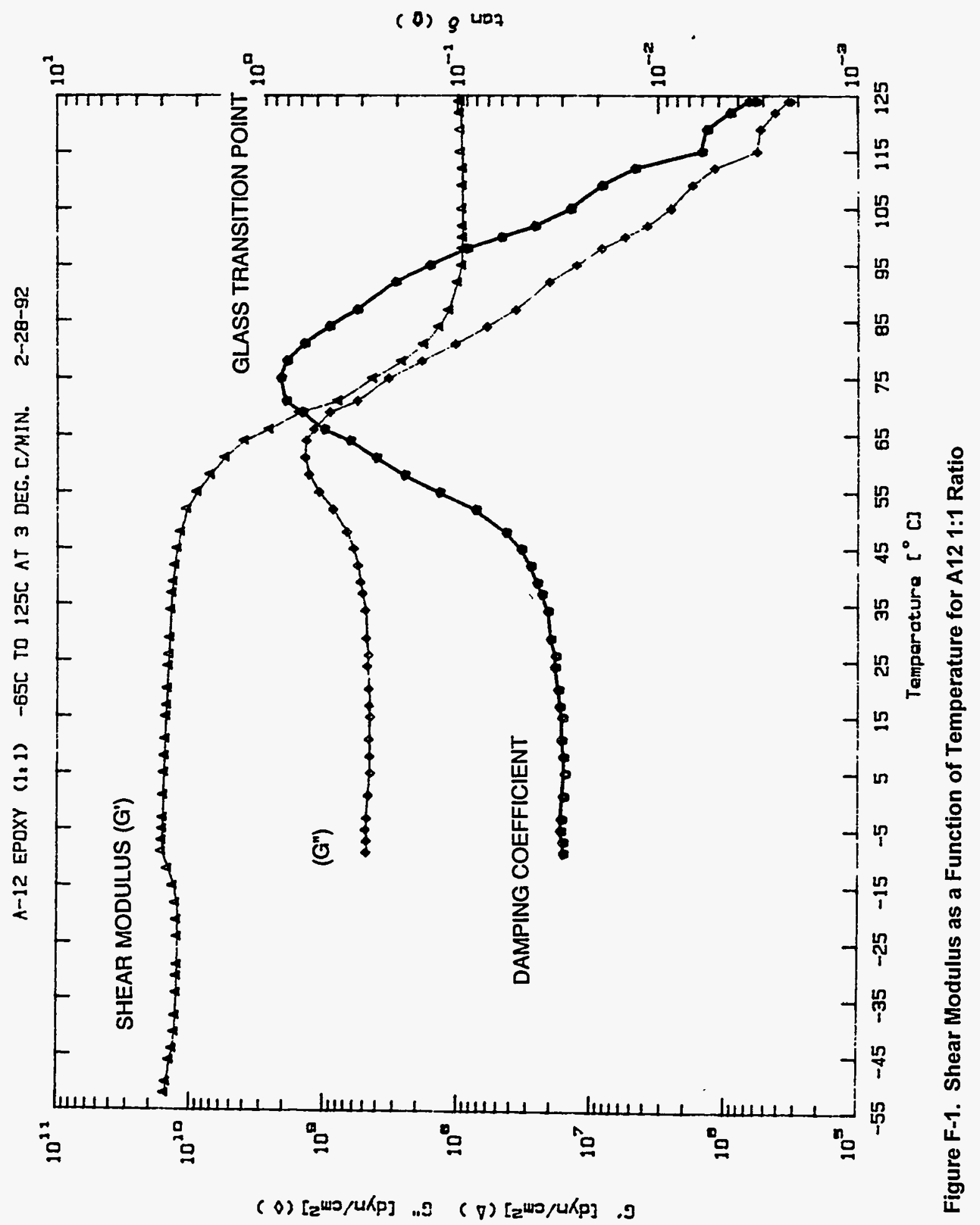




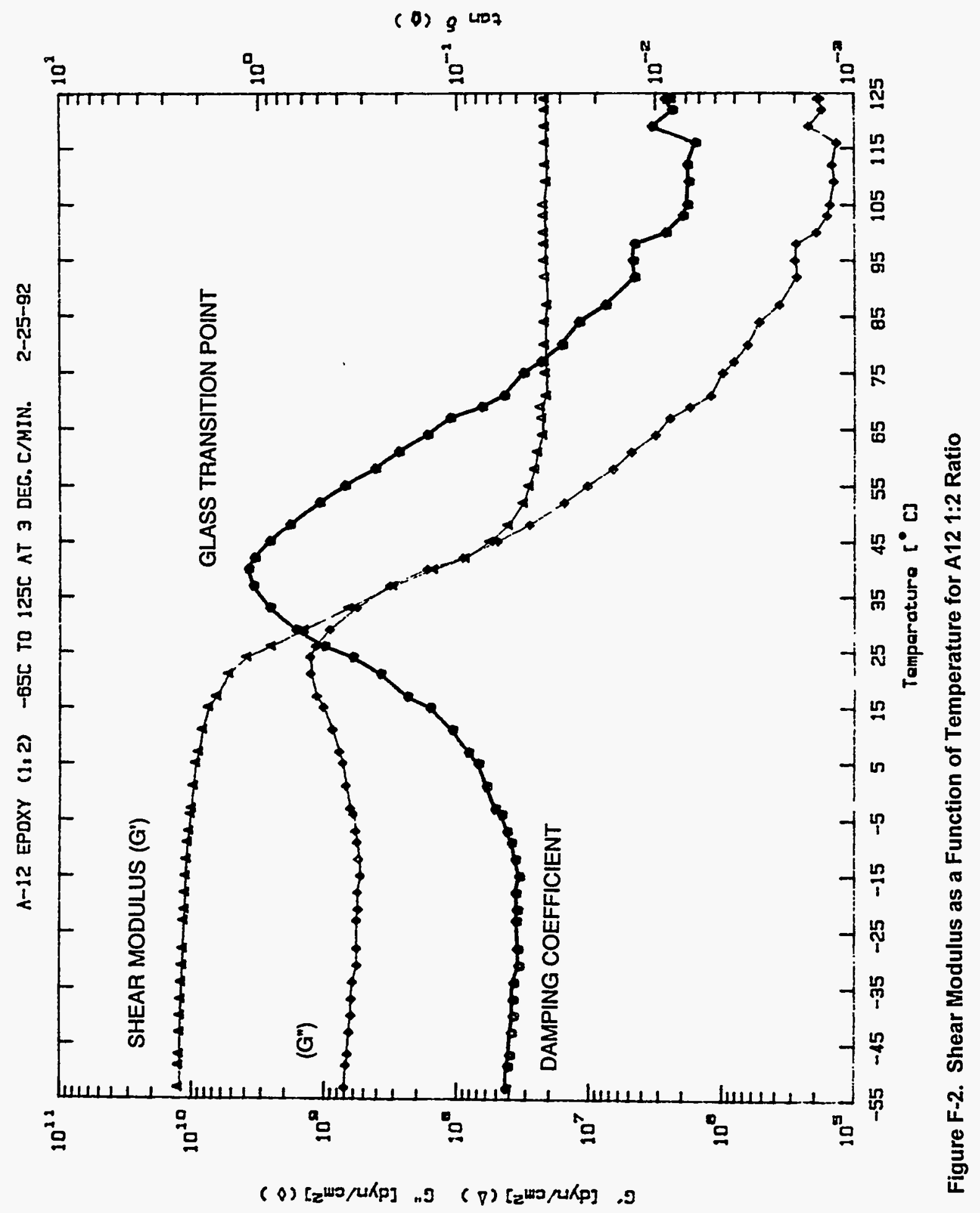




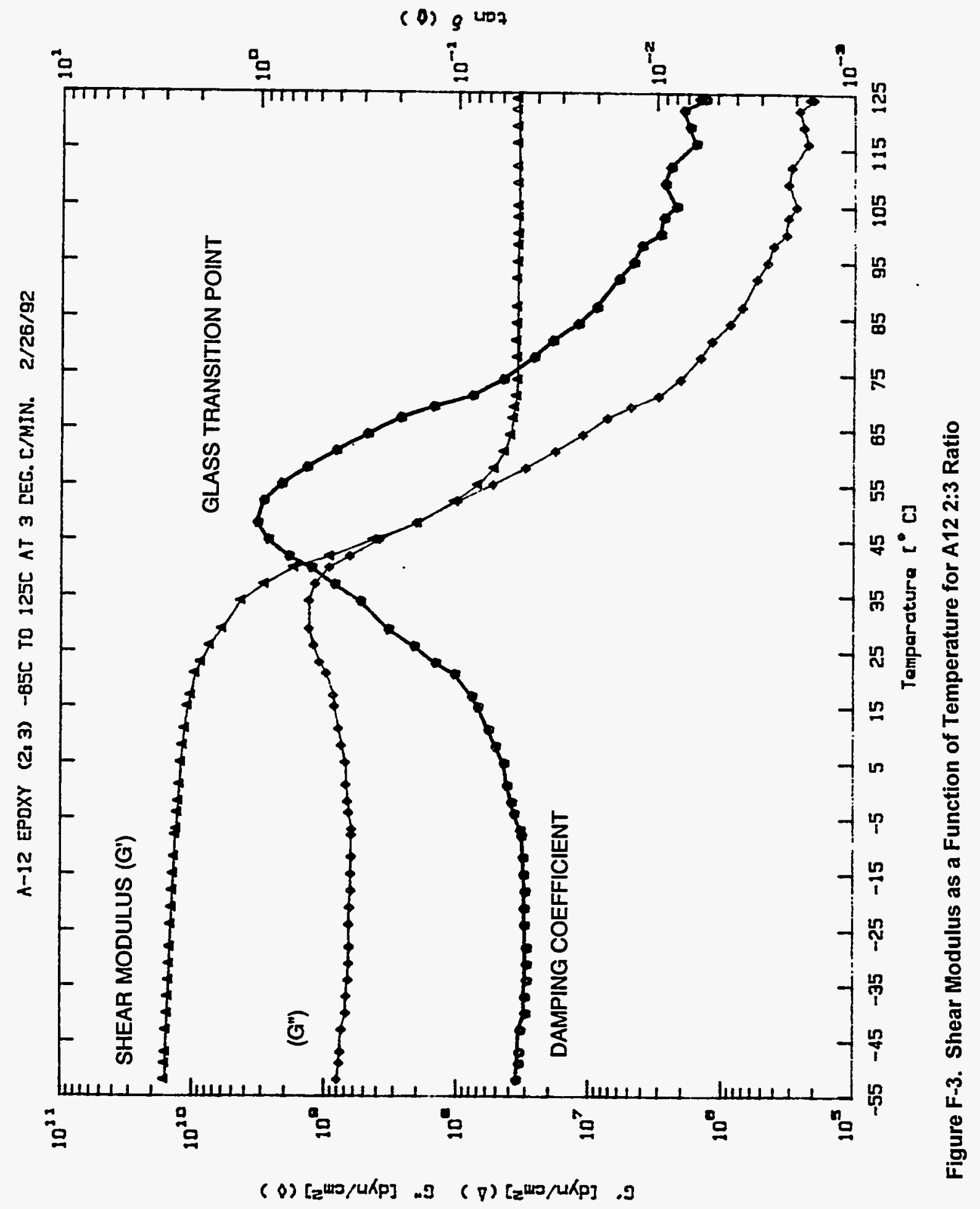




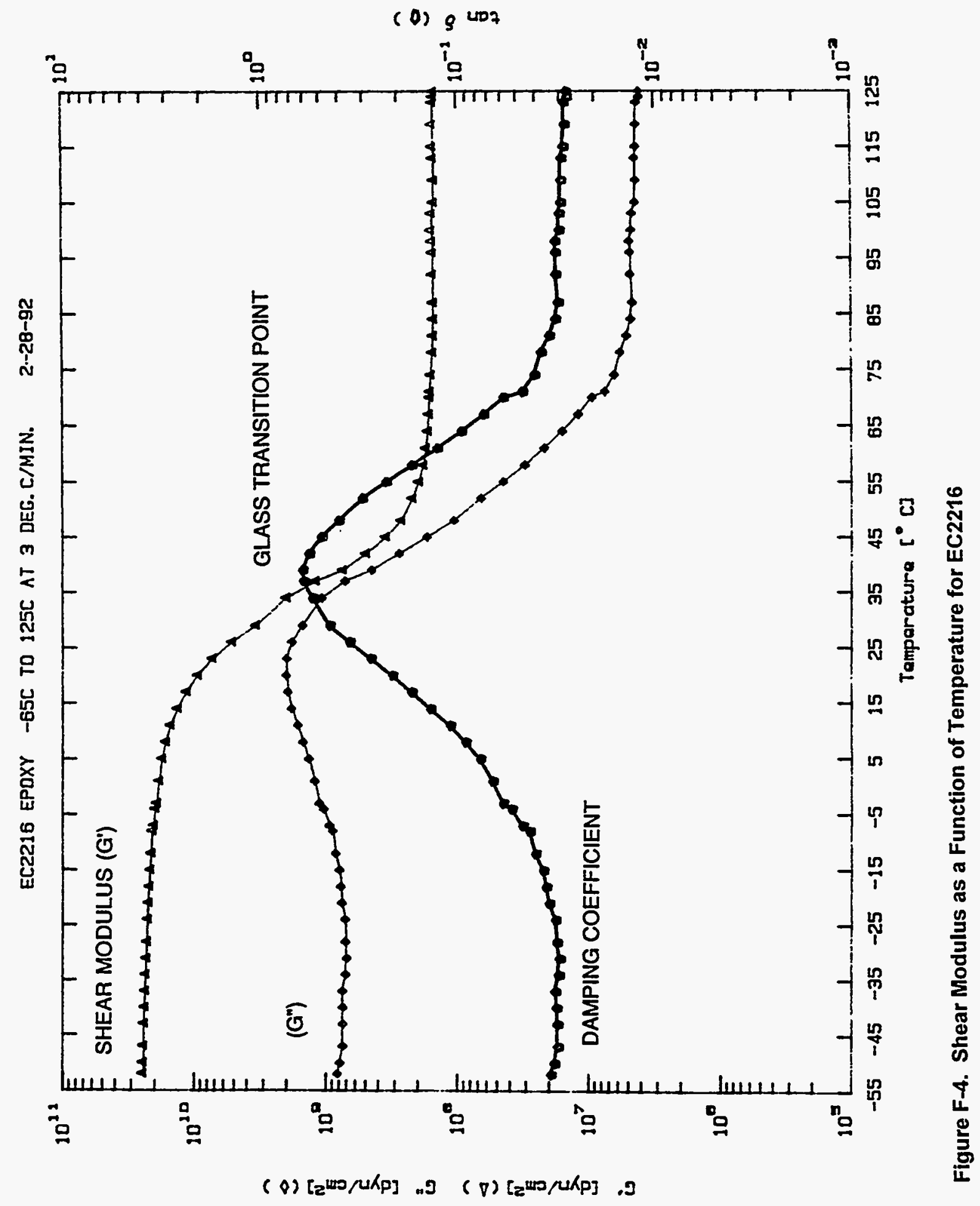




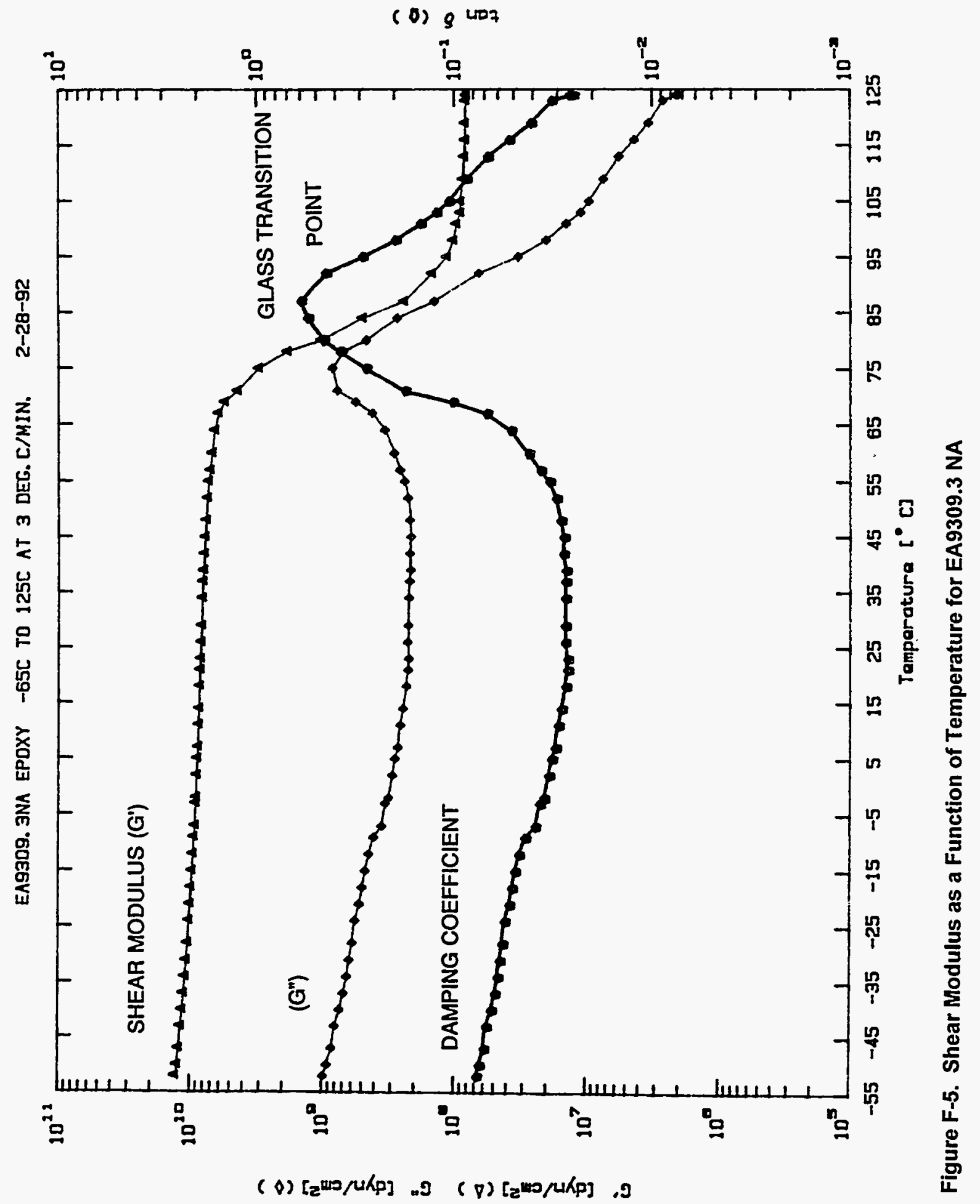


Table F-2. RMS Recorded Data for A12 (1:1)

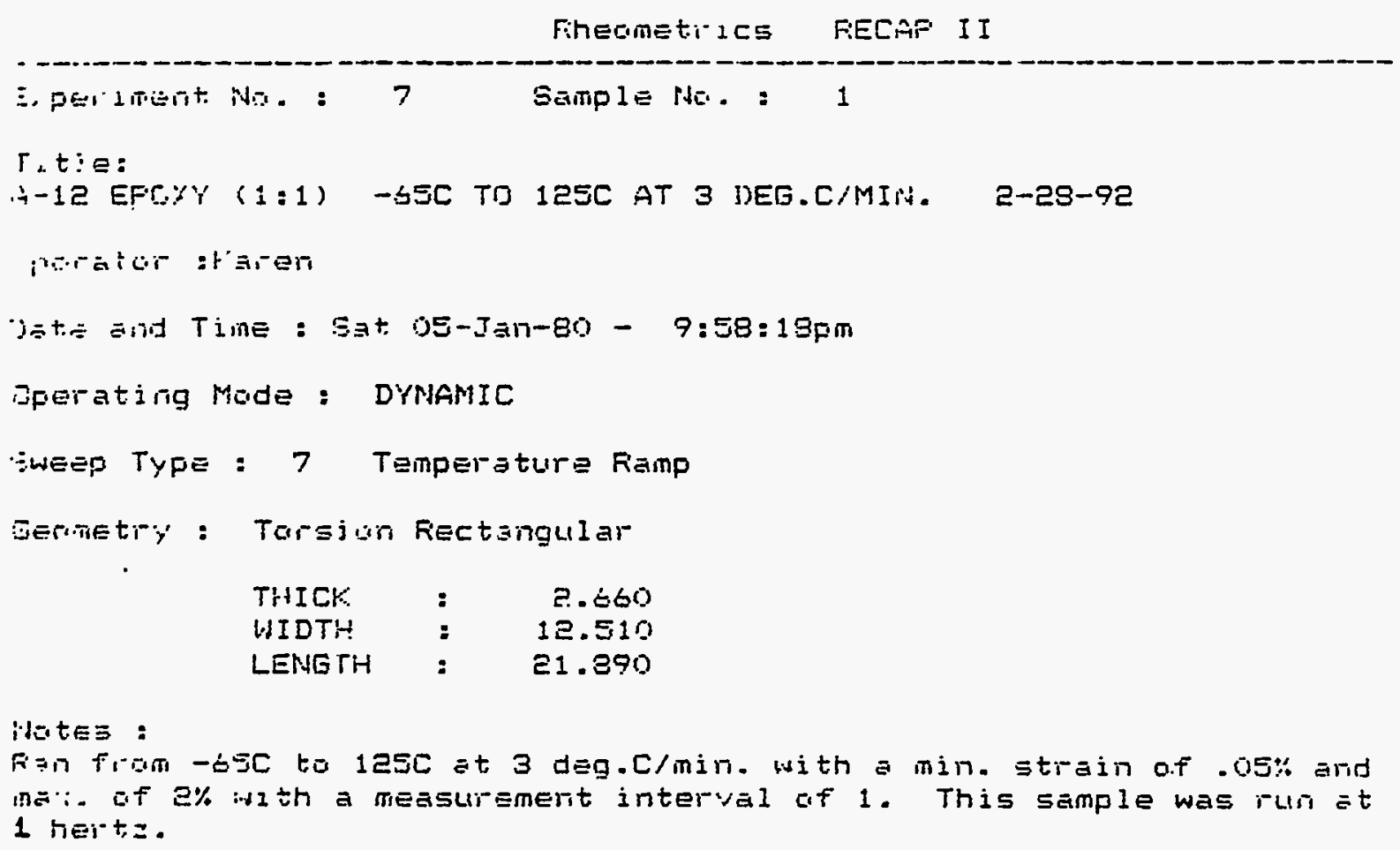


A-12 EFDXY (1:1) -E5C TO 125C AT 3 DEG.CIMIH. 2-2B-72

\begin{tabular}{|c|c|c|c|c|c|c|}
\hline 140. & $G^{*}$ & $G^{\prime \prime}$ & TANI_DEI_TA & \%STRAIN & TIHE & TEMF \\
\hline & d: & dyn/cm.sq. & & & min. & dEgreEE $=$ \\
\hline 1 & $1.725 e+10$ & $0.000=+00$ & $0.000 e+00$ & $4.861 e-02$ & 0.0 & -55.0 \\
\hline 2 & $1.7 \Xi 3 \mathrm{E}+10$ & $0.000 e+00$ & $0.000 \equiv+00$ & $4.864 e-02$ & 1.0 & -62.? \\
\hline 3 & $1.679 a+10$ & $0.000 e+00$ & $0.000 E+00$ & $4.865 e-02$ & 2.0 & $-57 . i$ \\
\hline 4 & 1. $579 E+10$ & $0.000 e+00$ & $0.000=+00$ & $4.873 e-02$ & 3.0 & $-5 \dot{0} 0$ \\
\hline 5 & i. Solle+10 & $0.000=+00$ & $0.000 e+00$ & $4.882 e-02$ & 4.0 & -52.0 \\
\hline$b$ & $1.455 E+10$ & $0.000: e+00$ & $0.000=+00$ & $5.876 e-02$ & 5.0 & $-5(\%, \therefore)$ \\
\hline 7 & $1.376 E+10$ & $0.000 e+00$ & $0.000 e+00$ & $5.883 e-02$ & 6.0 & -4.6 .0 \\
\hline 3 & $1.307 E+10$ & $0.000 e+00$ & $0.000=+00$ & $5.887 e-02$ & 7.0 & -44.0 \\
\hline 9 & 1. $2 \in \dot{E} e+10$ & $0.000 e+00$ & $0.000 e+00$ & $5.894 e-02$ & 8.0 & $-\dot{4} 1.9$ \\
\hline 10 & $1.247 e+10$ & $0.000 e+00$ & $0.000 e+00$ & $7.044 \mathrm{e}-0 \mathrm{e}$ & 9.0 & -38.0 \\
\hline 11 & 1. . $22 \beta e+10$ & $0.000 e+00$ & $0.000 E+00$ & $7.045 e-02$ & 10.0 & $-3<k$ \\
\hline 12 & 1. $220 \mathrm{e}+10$ & $0.000 e+00$ & $0.000 e+00$ & $7.045 e-02$ & 11.0 & $-31 \cdot 6$ \\
\hline 13 & 1.21RE+10 & $0.000 e+00$ & 0.000 etoo & $7.046 e-02$ & 12.0 & -29.0 \\
\hline 14 & 1.214e+10 & $0.000 e+00$ & $0.000 E+00$ & $7.046 \mathrm{e}-0 \mathrm{e}$ & 13.0 & -24.0 \\
\hline 15 & 1. $225 E+10$ & $0.000 e+00$ & 0.000 e+00 & $7.044 \mathrm{e}-0 \mathrm{e}$ & 14.0 & -21.0 \\
\hline 16 & $1.254 e+10$ & $0.000 e+00$ & $0.000 e+00$ & $7.041 \mathrm{e}-0 \mathrm{e}$ & 15.0 & -18.0 \\
\hline 17 & $1.313 E+10$ & $0.000 e+00$ & $0.000 e+00$ & $7.036 e-02$ & 16.0 & -15.0 \\
\hline 13 & $1.447 E+10$ & $0.000 e+00$ & $0.000 \leq \div 00$ & $7.021 e-02$ & 17.0 & -12.0 \\
\hline 17 & $1 . \$ 13 e+10$ & 4.76ńe+08 & $2.755 e-02$ & $7.000 e-02$ & 18.0 & -7.0 \\
\hline 20 & $1.597 \mathrm{e}+10$ & $4.748 e+09$ & $2.973 E-02$ & $7.000 e-02$ & 19.0 & -7.0 \\
\hline 21 & $1.581 E+10$ & 4. - 825e+08 & $3.053 e-02$ & $7.003 e-02$ & 20.0 & -5.0 \\
\hline 22 & $1.565 e+10$ & $4.733 e+08$ & 3.01BE-02 & $7.005 e-02$ & 21.0 & -3.0 \\
\hline$? 3$ & 1. $553 \varepsilon+10$ & $4.594 e+08$ & $2.757 \equiv-02$ & $7.006=-02$ & 2e. 0 & 1.0 \\
\hline 24 & $1.537 e+10$ & $4.456 e+08$ & $2.899=-02$ & $7.010 e-02$ & 23.0 & 5.0 \\
\hline 25 & 1. 522e+10 & $4.498 E+08$ & $2.955 e-02$ & $7.013 e-02$ & 24.0 & 8.0 \\
\hline PS & $1.508 e+10$ & 4.55'́e+08 & 3.021E-0D & $7.013 e-c 12$ & 25.0 & 11.0 \\
\hline 27 & 1. $48 B E+10$ & $4.447 e+08$ & $2.987 e-02$ & $7.015=-02$ & 26.0 & 15.0 \\
\hline $2 a$ & $1.46 \% e+10$ & $4.536 e+08$ & 3. $083 e-02$ & $7.018 \mathrm{e}-02$ & 27.0 & 17.0 \\
\hline 29 & $1.451 e+10$ & $4.562 e+08$ & $3.144 E-O D$ & $7.020 e-02$ & 28.0 & 20.0 \\
\hline 30 & $1.434 e+10$ & $4.684 e+08$ & $3.267 e-02$ & 7. $021 \mathrm{e}-0 \mathrm{z}$ & 29.0 & 24.0 \\
\hline 31 & $1.414 e+10$ & $4.593 e+08$ & $3.248 z-02$ & $7.026 e^{-02}$ & 30.0 & 26.0 \\
\hline $3 e$ & $1.393 E+10$ & $4.781 e+08$ & $3.432 e-02$ & 7.029e-02 & 31.0 & 29.0 \\
\hline 33 & 1.369e+10 & $4.865 \mathrm{e}+08$ & $3.554=-02$ & $7.030 e-02$ & 32.0 & 34.0 \\
\hline 34 & $1.342 E+10$ & $5.115 e+08$ & $3.810 \mathrm{e}-0 \mathrm{e}$ & $7.032 e-02$ & 33.0 & 37.0 \\
\hline 35 & $1.313 e+10$ & $5.300 e+08$ & $4.037 e-02$ & $7.038 e-02$ & 34.0 & 39.0 \\
\hline as & 1. $278 e+10$ & $5.542 e+08$ & $4.336 e-02$ & $7.042 \mathrm{e}-0 \mathrm{e}$ & 35.0 & 42.0 \\
\hline 37 & 1. $232 e+10$ & $5.965 E+08$ & $4.844 e-02$ & $7.048 \mathrm{e}-0 \mathrm{e}$ & 36.0 & 45.0 \\
\hline 39 & $1.164 E+10$ & $\therefore .722 e+0 s$ & $5.777 e-02$ & $7.058 e-02$ & 37.0 & 48.0 \\
\hline 37 & $1.04 E e+10$ & $8.530=+08$ & $8.186 e-02$ & $7.073 e-02$ & 38.0 & SE. 0 \\
\hline 40 & $8.761 E+09$ & $1.085 e+09$ & $1.237 e-01$ & 8. 528e-02 & 39.0 & 55.0 \\
\hline 41 & $6.7860+09$ & 1. $295=+09$ & $1.854 e-01$ & $1.019 e-01$ & 40.0 & 58.0 \\
\hline 42 & $5.395 e+09$ & $1.377 e+09$ & $2.555 e-01$ & 1. .225e-01 & 41.0 & 61.0 \\
\hline 43 & 3.392e+09 & $1.347 E+09$ & $3.467 e-01$ & $1.487 e-01$ & 42.0 & 64.0 \\
\hline 44 & 2.535e+09 & 1. 18Ee+09 & $4.667 e-01$ & $1.730 e-01$ & 43.0 & $\therefore \dot{0}$ (i) \\
\hline 4.5 & $1.497 \mathrm{e}+09$ & $8.972=+08$ & $5.985 e-01$ & e. $137 e-01$ & 44.0 & $\therefore 0$ \\
\hline 46 & $7.707 \pm+01$ & $5.582 e+08$ & $7.243 e-01$ & $2.575 e-01$ & 45.0 & $=1.0$ \\
\hline 47 & $4.246 a+08$ & 3.260 $=+108$ & $7.693 \mathrm{e}-01$ & $3.091 e-01$ & 46.0 & 75.0 \\
\hline 48 & $2.571 \in+08$ & i. $8390+08$ & $7.152 x-01$ & $3.716 e-01$ & 47.0 & 75.0 \\
\hline 4? & $1.762 E+08$ & $1.031 \equiv+08$ & $5.350 e-01$ & $4.459 e-01$ & 48.0 & 31.0 \\
\hline 50 & 1.35se+08 & $5.970 z+07$ & $4.403 e-01$ & $5.351 E-01$ & 49.0 & 34.0 \\
\hline
\end{tabular}




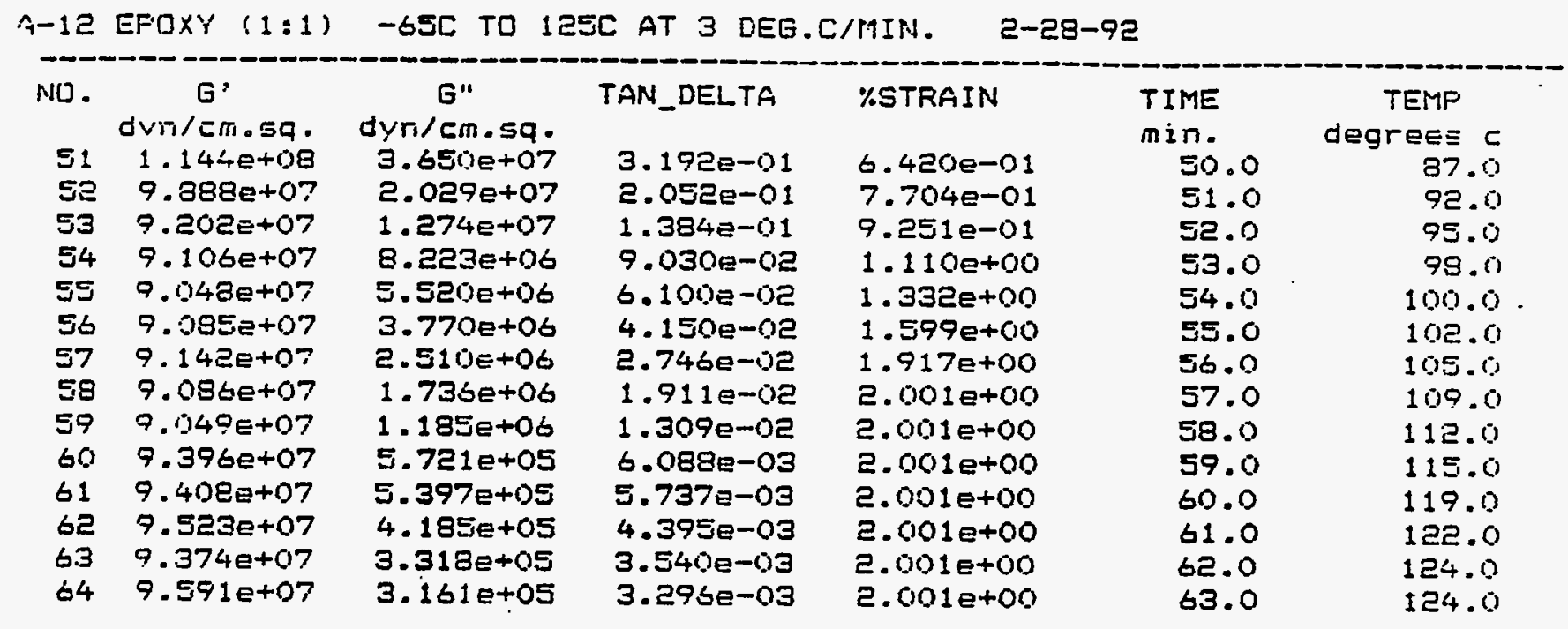


Table F-3. RMS Recorded Data for A12 (1:2)

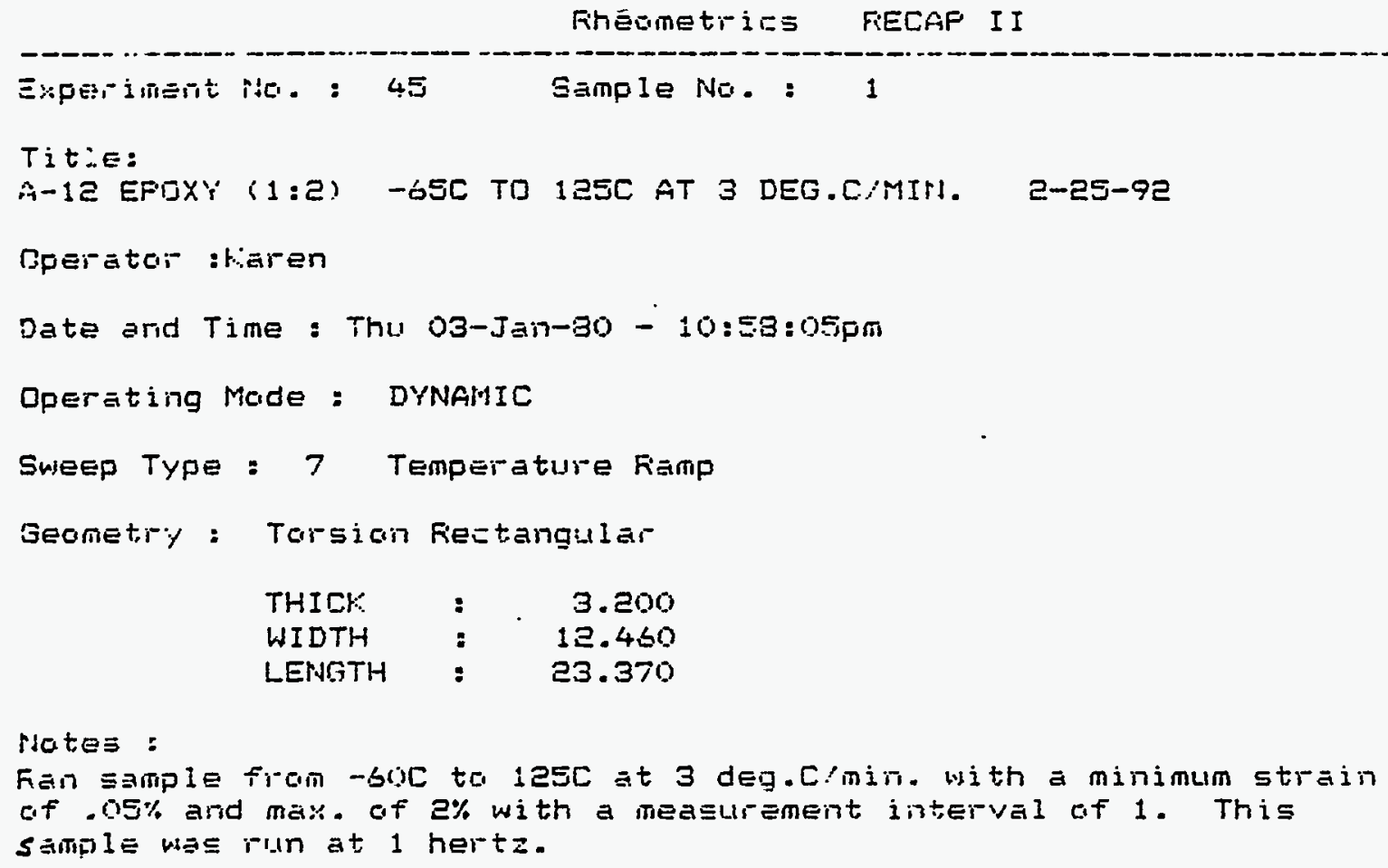


i-1E EFOXY (1:E) -6EC TO $125 C$ AT 3 DEG.CIMIN. 2-25-72

\begin{tabular}{|c|c|c|c|c|c|c|}
\hline isa. & $\begin{array}{c}G^{*} \\
d y n / c \pi_{1} .5 q .\end{array}$ & $\begin{array}{c}\text { G" } \\
\text { dyn/cm.sq. }\end{array}$ & TAM_DELTA & \%STRAIN & $\begin{array}{l}\text { TIME } \\
\text { min. }\end{array}$ & $\begin{array}{c}\text { TERTF } \\
\text { dEQREES }=\end{array}$ \\
\hline 1 & $1.261 e+10$ & $7.777 e+08$ & $6.16 \leqslant E-O D$ & 4.653e-02 & 0.0 & -55.0 \\
\hline 2 & 1. $251 e+10$ & $7.439 e+08$ & $5.877 e-02$ & $4.652 E-02$ & 1.0 & - \\
\hline 3 & 1. . $254 E+10$ & $7.385 e+08$ & $5.871 e-02$ & $4.652 e-02$ & 2.0 & -50.6 \\
\hline 4 & $1.245 e+10$ & $7.2719+08$ & $5.842 e-02$ & $4.653 e-02$ & 3.0 & $-5=.0$ \\
\hline 5 & 1. $23 \rightarrow E+10$ & $7.012 e+03$ & $5.682 E-02$ & $4.6565-02$ & 4.0 & -52.0 \\
\hline$\therefore$ & 1. EESE+10 & $6.810 e+08$ & $5.560 e-02$ & $4.057 e-02$ & 5.0 & -47.0 \\
\hline 7 & 1.210E+10 & $6.611 e+08$ & $5.437 \varepsilon-02$ & $4.660 \mathrm{e}-0 \mathrm{E}$ & 0.0 & -47.0 \\
\hline 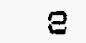 & 1. $205=+10$ & $6.428 E+08$ & 5.336́e-02 & $4.6629-02$ & 7.0 & -43.0 \\
\hline$\%$ & $2.192 e+10$ & $6.221=+08$ & $5.217 \in-02$ & $4.364 e-0 e$ & 8.0 & -40.0 \\
\hline 10 & $1.181 .+10$ & $6.180 e+03$ & $5.231=-02$ & $4.663 e-02$ & 9.0 & -37.0 \\
\hline 11 & $1,168 \mathrm{e}+10$ & 6. $05 \dot{5}$ +0a & $5.187 e-02$ & $4.666 e-02$ & 10.0 & -34.0 \\
\hline 12 & $1.150 z+10$ & $5.627 e+08$ & $4.893 e-02$ & $5.788 e-02$ & 11.0 & $-3: 0$ \\
\hline 13 & 1.1.36e+10 & $5.637 e+08$ & $4.963 e-02$ & $5.788 e-02$ & 12.0 & -23.0 \\
\hline 14 & $1.121 E+10$ & $5.601 \mathrm{e}+09$ & $5.052 e-02$ & $5.791 \mathrm{e}-02$ & 13.0 & -23.0 \\
\hline 15 & $1.100 e+10$ & 5.525et08 & $4.994 \equiv-02$ & $5.792 e-02$ & 14.0 & -21.0 \\
\hline $1 . \Rightarrow$ & $1.973 e+10$ & $5.575 e+08$ & $5.079 e-02$ & $5.794 a-02$ & $i 5.0$ & -13.0 \\
\hline 17 & $1.089 E+10$ & $5.312 e+08$ & $4.879=-02$ & $5.797 e-02$ & 16.0 & -15.0 \\
\hline 18 & $1.073 e+10$ & $5.494=+09$ & $5.122 e-02$ & $5.798 E-02$ & 17.0 & -12.0 \\
\hline 19 & $1.054 e+10$ & $5.640 e+08$ & $5.350 \in-02$ & $5.903 e-02$ & 18.0 & -9.9 \\
\hline 20 & $1.031 E+10$ & $5.821 \in+08$ & $5.6465-02$ & $5.307 e-02$ & 19.0 & -7.0 \\
\hline 21 & $1.007 e+10$ & S.040e+08 & $5.998 \varepsilon-02$ & $5.311 \mathrm{e}-0 \mathrm{e}$ & 20.0 & -4.0 \\
\hline 22 & $9: 867 E+09$ & $6.333 e+08$ & $6.474 E-02$ & $5.313 e-02$ & 21.0 & -3.0 \\
\hline ej & $7.534 e+09$ & j.356e+08 & $7.153 E-02$ & $5.318 e-02$ & 22.0 & 1.0 \\
\hline 24 & O. $228 E+09$ & $7.292 e+09$ & 7.702 e -02 & $5.324 e-02$ & 23.0 & 5.0 \\
\hline 25 & $8.752 E+09$ & $7.745 e+08$ & $3.349 e-02$ & $7.110 \mathrm{e}-02$ & 24.0 & 7.0 \\
\hline$=6$ & $9.193 e+09$ & $8.731 e+09$ & $1.06 \leqslant=-0:$ & $7.124 e-02$ & 25.0 & 11.0 \\
\hline$\Xi 7$ & $7.379 e+09$ & $1.018=+07$ & $1.380 \in-01$ & $7.141 e-02$ & 26.0 & $1 \equiv .0$ \\
\hline $2 \cdot 3$ & $5.401=1.00$ & $1.14 .6=09$ & $1.771 \leq-01$ & $8.461=-02$ & 27.0 & 17.0 \\
\hline 30 & $5.1 \div 5 E+09$ & $1.2670+09$ & 2.44ae-01 & 1.02se-01 & 28.0 & 21.0 \\
\hline 30 & $3.806 a+09$ & 1. $281 E+07$ & $3.367 \equiv-01$ & $1.231 e-01$ & 29.0 & 2.4 .0 \\
\hline$\because$ & 2. $4 E$ EE +00 & $1.163 e+09$ & $4.634 e-01$ & $1.480 e-01$ & 30.0 & 26.0 \\
\hline 32 & $1.37:=e+07$ & $9.094 e+08$ & $6.513 \equiv-01$ & $1.794 e-01$ & 31.0 & 29.0 \\
\hline $3: 3$ & $3.39: e+n g$ & $5.635 e+09$ & $8.313 e-01$ & 2. $146 e-01$ & 32.0 & 33.0 \\
\hline 34 & F. PECR+OB & 3.18.ذE+0E & $1.067 E+00$ & $2.576 \mathrm{e}-01$ & 33.0 & 37.0 \\
\hline$\because$ & i. $\quad+\cdots$ & $\therefore \therefore \therefore=706$ & $1.130 e+00$ & $3.101 e-01$ & 34.0 & 40.0 \\
\hline$?$ & $? \cdot-1 \cdot 1=+7$ & $=.90^{-}=+\div$ & $1.051+3$ & $\exists .713 e-01$ & 35.0 & $42 . \therefore$ \\
\hline- & $\therefore \therefore=r$ & $\therefore \angle 0=+07$ & $3.915 \cdots$ & $\rightarrow 4=-9 !$ & 36.0 & 45.0 \\
\hline זִד & $\because \cdots \because 7$ & :! $31^{\circ}=16$ & $\therefore 77: \infty$ & $=.352=-191$ & 37.0 & $\because^{-}$ \\
\hline$\because \because$ & $\because \because \because 87$ & $1.58: 16 \%$ & $4.94_{1}=-\therefore 1$ & $\therefore \quad \| P_{4}$ & 33.6 & $\because$ \\
\hline & $\because \therefore: 1:-17 ?$ & $1.031=07=$ & 马.SE!: & $-\quad \because \bar{\gamma}^{-}=-01$ & ר & $\because$ \\
\hline$\because$ & $\because .53^{\circ}=+07$ & S.575.7+n! & $\equiv .55 \cdot \therefore:$ & $r \cdot$. ZE SE -.1! & $-i .0$ & $\cdots$ \\
\hline $4 a$ & ? & 4.77:ee+0s & $1.975=-01$ & $1: 090+00$ & $\therefore 1,0$ & · i i \\
\hline 43 & 2.2:1อ+07 & 3.13Eetot & $1.45 \div \cdots:$ & $1.3300+00$ & $4 \equiv .0$ & \\
\hline 44 & 2.24ise+o7 & 2. $443 z+06$ & $1.088=-01$ & $1.597 e+00$ & $.3 .$. & $\therefore \therefore$ \\
\hline $4 \div$ & 2.259E+07 & $1.7300+06$ & 7.54 se-0. & $1.719 e+00$ & 44.0 & $\therefore$ \\
\hline$\div 6$ & ₹.065e+07 & $1.20 .5=+01$ & $5.839 E-02$ & $2.001 e+00$ & 45.0 & -1.0 \\
\hline 47 & $2.1110+07$ & $7.364 e+05$ & 4. $645 E-0 R$ & $2.001 e+00$ & 45.0 & 75.0 \\
\hline 43 & $2.13^{7}=07$ & $9.077 \Leftrightarrow+05$ & 3.790E-02 & $2.001=000$ & 47.0 & $7-. .1$ \\
\hline 49 & 2. $140 E+07$ & $6.26 ? e+05$ & $2.773 e-0$ & $2.001 e+00$ & 48.0 & 80.0 \\
\hline 50 & $2.137 E+07$ & $5.204=+05$ & 2.436=-62 & $2.001 E+00$ & +9.0 & 34.0 \\
\hline
\end{tabular}




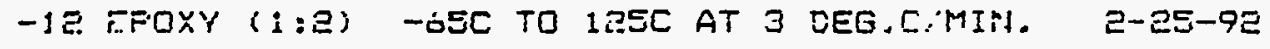

\begin{tabular}{|c|c|c|c|c|c|c|}
\hline ilC: & $6^{\circ}$ & $\mathbf{G}^{\prime \prime}$ & TAR__DELTA & $\because$ ETFAIN & TIME & TEHIF \\
\hline 51 & 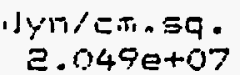 & $\begin{array}{l}\text { dyn/cin. } 5 q . \\
3.671 .0+0.5\end{array}$ & $1.792=-02$ & $2.001 e+00$ & $\begin{array}{l}\min \\
50.0\end{array}$ & $\begin{array}{r}\text { dEgrEES } ᄃ \\
3 ? .0\end{array}$ \\
\hline 5 & $2.11=e+07$ & E.71.5E+05 & 1. $2 B 2=-02$ & $2.0012+00$ & 51.0 & 72.9 \\
\hline 53 & E.15\}e+0? & $2.807_{e+05}$ & $1.306=-0 \%$ & $2.001 \mathrm{e}+00$ & 도. 0 & 75.0 \\
\hline 54 & 2. $1+2 E+07$ & $2.7495+05$ & 1. $23 i z e-O 2$ & $2.001 e+00$ & 53.0 & 93.0 \\
\hline $5:$ & $\therefore$ is $\leq 4 e+07$ & 1. $928 E+05$ & $8.911 E-03$ & e. $.001 \mathrm{E}+60$ & 54.0 & $10(0.0$ \\
\hline 56 & 2. $179 \equiv+07$ & 1.595E+05 & $7.324 E-03$ & $2.001 e+00$ & 55.0 & 103.0 \\
\hline 57 & 2. 17FE+07 & $1.5162+05$ & $6.959 e-03$ & $2.001 \mathrm{e}+00$ & 56.0 & 105.0 \\
\hline 稞 & 2.06ㄷ+07 & $1.415 E+05$ & $6.836 e-03$ & $2.001 e+00$ & 57.0 & 100.0 \\
\hline $5 ' 7$ & E. $10 t e+07$ & $1.465 e+05$ & $6.953 e-03$ & 2. $001 \mathrm{e}+00$ & 58.0 & 112.0 \\
\hline o & อ. 12Еニ+ & $1.347 \equiv+105$ & $6.348 e-03$ & $2.001 e+00$ & 59.0 & 116.0 \\
\hline 61 & 2.116e+07 & 2. $208 E+05$ & $1.044 e-02$ & $2.001 e+c 0$ & 60.0 & 117.0 \\
\hline בקמם & 2. $114 E+07$ & $1.748 E+05$ & $8.2702-03$ & $2.001 e+00$ & 61.0 & 122.0 \\
\hline$\dot{\Delta}$ & 2.116e+07 & 1.892e+05 & $8.942 e-03$ & $2.001 e+00$ & s2. 0 & 124.0 \\
\hline 34 & 2. 127Е+07 & $1.804 E+05$ & $8.484 e-03$ & $2.001 e+00$ & 63.0 & 124.6 \\
\hline
\end{tabular}


Table F-4. RMS Recorded Data for A12 (2:3)

Fheametirics REDAF II

E:perirent $\mathrm{Ha}$ : 4 í Sample $\mathrm{Ma}$ : 1

Title:

คิ-12 EFOXY !2:3; -S5C TO 1ESC AT 3 DEG.C/MIM. 2/26/92

Boerstai : Karen

liate and Time : Fri 04-Jan-80j-10:10:32pm

Dperating Made : DYMAMIC

SWEEP TYPE : 7 TEmpEratui= Fiant

Gi=onetry : TorEian FeEtangular

$\begin{array}{llr}\text { THICH } & : & 3.240 \\ \text { WIDTH } & : & 12.520 \\ \text { LENGTH } & : & 24.000\end{array}$

Neites:

This simple was ruin from -s.se to 1250 at 3 deg.c/min. with a min. Etrain of . $5 \%$ and $m a \%$. of $\Xi \%$ with a messirement interval of 1 .

rile $\equiv$ infle uas jun at 1 hertz. 


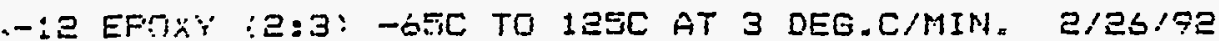

\begin{tabular}{|c|c|c|c|c|c|c|}
\hline$\therefore 10$ & $\mathbf{G}^{\circ}$ & $G^{\prime \prime}$ & TAN_DELTA & \%STFAIN & TIME & TEHF \\
\hline & 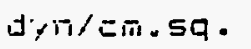 & dyr/cm sq. & & & min. & 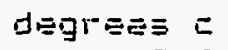 \\
\hline 1 & 1.6sie+1.j & $3.543=+03$ & $5.144=-62$ & $4.494 e-02$ & 0.0 & $-65 . \therefore$ \\
\hline 2 & $1.645=+1.3$ & $8.685 E+08$ & $5.273 e-02$ & $4.696 e-02$ & 1.0 & $-\infty 1.0$ \\
\hline 3 & 1. SEGE+10 & $8.464 E+0 \theta$ & $5.205=-02$ & $4.700 e-02$ & 2.0 & -57.0 \\
\hline 4 & 1.50हE+10 & $5.105 e+00$ & $5.058=-02$ & $4.705 e-02$ & 3.0 & -55.9 \\
\hline 5 & $1.58 r e+10$ & $7.934 \varepsilon+08$ & $5.015=-02$ & $4.707 e-0 e$ & 4.0 & -52.5 \\
\hline 6 & 1. 562e+10 & $7.627 \in+08$ & 4.88'te-oz & $4.709 e-02$ & 5.0 & -49.0 \\
\hline 7 & $1.541 E+10$ & $7.480 e+08$ & $4.353 \equiv-02$ & $4.711 e-02$ & 6.0 & -47.0 \\
\hline 8 & 1. $519=+10$ & $7.297 e+08$ & $4.804 E-02$ & $4.713 e-02$ & 7.0 & -43.0 \\
\hline 9 & 1. $478 \mathrm{Be}+10$ & $\dot{0.784 E+08}$ & 4.529E-02 & $4.717 e-02$ & 8.0 & -40.0 \\
\hline 10 & $1.4800+10$ & $6.7300+08$ & $4.547 e-12$ & $4.719 e-02$ & 9.0 & -37.0 \\
\hline 11 & $1.462 e+10$ & $6.507 e+08$ & $4.451 \equiv-02$ & $4.721 e-02$ & 10.0 & -34.0 \\
\hline 12 & $1.445 e+10$ & $6.432 E+08$ & $4.451=-02$ & 4. $723 e-02$ & 11.0 & -31.0 \\
\hline 13 & 1. $42 B \mathrm{E}+10$ & $6.363 E+08$ & $4.456 e-62$ & 4. $727 e-02$ & 12.0 & -28.0 \\
\hline $1 \div$ & $1.40 \% e+10$ & $0.427 \mathrm{E}+0 \mathrm{~B}$ & $4.568 E-02$ & $4.728 e-02$ & 13.0 & -24.0 \\
\hline 15 & 1. $3 a r: e+10$ & $\therefore .34 \mathrm{~B} \equiv+0 \mathrm{~B}$ & $4.572=-02$ & $4.731 e-02$ & 14.0 & -21.0 \\
\hline 16 & 1.372E+10 & 6.227E.+08 & $4.539 e-02$ & $4.732=-02$ & 15.0 & $-18 \cdot 0$ \\
\hline $1 \%$ & 1. $353 E+10$ & D. $244 \mathrm{e}+05$ & $4 . \$ 15 E-02$ & $4.735 e-02$ & 16.0 & -15.0 \\
\hline 13 & $1.332=+10$ & $6.174 E+09$ & $4.531 \equiv-02$ & $4.739 e-02$ & 17.0 & -12.0 \\
\hline 17 & $1.31 \pm e^{2}+10$ & $6.195 \mathrm{E}+08$ & 4.725 $=-02$ & $4.743 e-02$ & 18.0 & -3.0 \\
\hline 8 & 1.2В6z+10 & 6.165E+0B & $4.795 E-02$ & $4.746 e-02$ & 17.0 & -7.0 \\
\hline 21 & 1. $26 z e+10$ & 1.497e+08 & $5.150 E-02$ & 4. $749 e-02$ & 20.0 & -4.0 \\
\hline 22 & 1. $243 E+10$ & $6.624 E+08$ & $5.330=-02$ & $4.751 e-02$ & 21.0 & -2.0 \\
\hline 23 & 1.:ZERE+10 & $6.841 e+08$ & $5.607 \mathrm{E}-02$ & $4.754 e-02$ & 22.0 & 1.0 \\
\hline 24 & $1.17 i E+10$ & $0.925 E+08$ & $5.817 \equiv-02$ & $4.759 e-02$ & 23.0 & 5.0 \\
\hline 25 & $1.159 e+10$ & $7.441 \equiv+0 B$ & $5.421 E-02$ & 4. $764 e-02$ & 24.0 & 5.0 \\
\hline 25 & $1.121 E+10$ & $7.813 \Xi+08$ & $6.969=-02$ & $4.763 e-02$ & 25.0 & 11.0 \\
\hline 27 & $1.067 e+10$ & 3.432e+os & $7.887 e-02$ & $4.774 e-02$ & 26.0 & 15.0 \\
\hline 28 & $1.010 E+10$ & 9. $545 \Xi+08$ & $8.464 \equiv-02$ & $5.987 e-02$ & 27.0 & 17.0 \\
\hline 27 & $9.339 e+09$ & $9.707 e+08$ & $1.034 e-01$ & $5.899 e-02$ & 28.0 & 21.0 \\
\hline 30 & $3.470 e+09$ & $1.097 E+09$ & $1.295=-01$ & $5.915 e-02$ & 29.0 & 23.0 \\
\hline 31 & 7. 299e+09 & 1.21 $2 \mathrm{e}+09$ & $1.660 \mathrm{e}-01$ & $7.046 e-02$ & 30.0 & 26.0 \\
\hline 32 & $5.894 e+09$ & $1.318 e+09$ & 2. $236=-01$ & $8.518 e-02$ & 31.0 & 59.6 \\
\hline 33 & $4.240 p+09$ & $1.315 e+09$ & $3.100 e-01$ & $1.015 e-01$ & 32.0 & 34.0 \\
\hline 34 & 2.830e+09 & $1.183 e+09$ & $4.181=-01$ & $1.233 e-01$ & 33.0 & 37.0 \\
\hline 35 & 1. 689 et09 & $7.267 e+08$ & $5.486 e-01$ & $1.479 e-01$ & 34.0 & 40.0 \\
\hline 36 & $7.094 E+03$ & $6.454 E+08$ & $7.121=-01$ & $1.788 e-01$ & 35.0 & 42.0 \\
\hline 37 & $4.244 e+0 B$ & 3.858e+08 & $7.091 e-01$ & 2. $150 \mathrm{e}-01$ & 36.0 & 45.0 \\
\hline 33 & 1. $? \leqslant B E+0 B$ & 2. .28E+08 & $1.030 E+00$ & 2. $573 e-01$ & 37.0 & 48.0 \\
\hline 37 & $1.034 a+08$ & $9.917 e+07$ & $9.589 e-01$ & $3.091 e-01$ & 38.0 & 52.0 \\
\hline 40 & $6.898=+07$ & $5.376 E+07$ & $7.794 E-01$ & $3.727 e-01$ & 39.0 & 5.5 .0 \\
\hline$\dot{4}+1$ & $5.316 E+07$ & $3.030 e+07$ & $5.810 e-01$ & $4.454 e-01$ & 40.0 & 53.0 \\
\hline 42 & 4.37GE+07 & $1.806 e+07$ & $4.127 e-01$ & $5.349 e-01$ & 41.0 & 61.0 \\
\hline 43 & $3.907 e+07$ & $1.121 \mathrm{e}+07$ & $2.369 E-01$ & $6.421 \mathrm{e}-01$ & 42.0 & 54.0 \\
\hline 44 & $3.749 e+07$ & $7.321 E+0 B$ & $1.953 \mathrm{e}-01$ & $7.703 e-01$ & 43.0 & 67.0 \\
\hline 4.5 & $3.557 e+07$ & $4.8672+06$ & $1.331 \equiv-01$ & $9.255 e-01$ & 44.0 & 69.0 \\
\hline 46 & $3.533 E+07$ & $3.009=+0 B$ & $3.518=-02$ & $1.111 e+00$ & 45.0 & 71.0 \\
\hline 47 & $3.467 e+67$ & 2.0144a+0s & $5.952 e-02$ & $1.331 e+00$ & 46.0 & 74.0 \\
\hline 43 & $3 .+72 e+67$ & $1.455=+06$ & $4.167 e-02$ & $1.592 E+00$ & 47.0 & 78.0 \\
\hline 47 & $3.555=+07$ & $1.172 e+0 s$ & 3.3느르-0? & $1.918 e+00$ & 49.0 & 31.0 \\
\hline 50 & $3.504=+07$ & $0.739 E+05$ & $2.494 E-02$ & $2.000 e+00$ & 49.0 & 84.0 \\
\hline
\end{tabular}




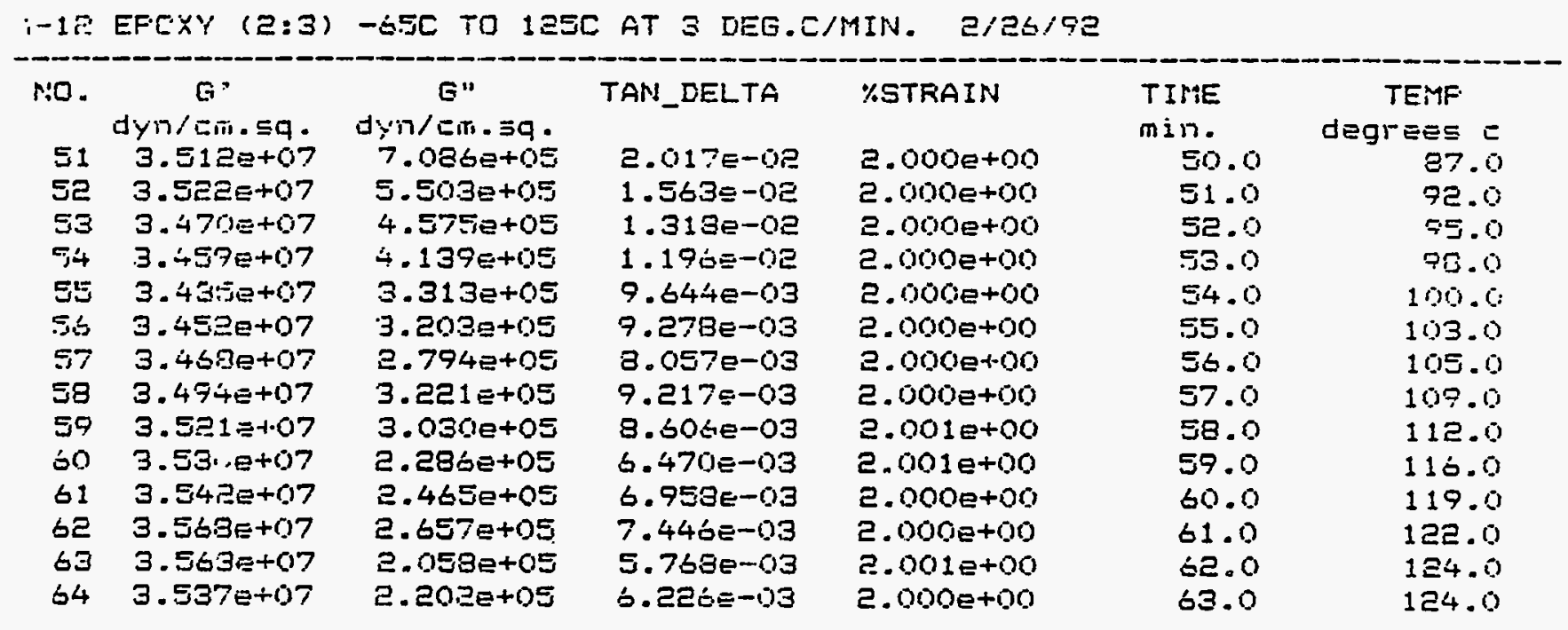


Table F-5. RMS Recorded Data for EC2216

Fheametrics F:EC.AF II

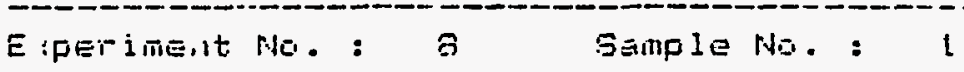

Tirile:

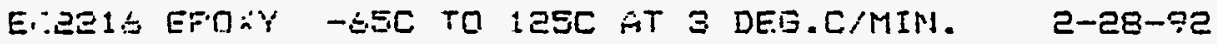

LFeratar :Kartir

$\Gamma \div t . E$ and $T i m e$ : Sat $05-J a n-80-11: 55: 46 \% m$

Opersting Made : DYMaMIC

EwE⿰氵P Type : 7 Temperatuie Fiamp

Grometiry : Torsian Fiectarigular

$\begin{array}{llr}\text { THICH } & : & 2.570 \\ \text { WIDTH } & : & 12.490 \\ \text { LENIETH } & : & 24.130\end{array}$

Hetes :

Fian sample from $-65 \mathrm{C}$ to $125 \mathrm{C}$ at 3 deg.C/min. with a min. strain of $.05 \%$ and mai. of $2 \%$ with a neasurement interval af 1 . This sample (was run at 1 hEitz. 


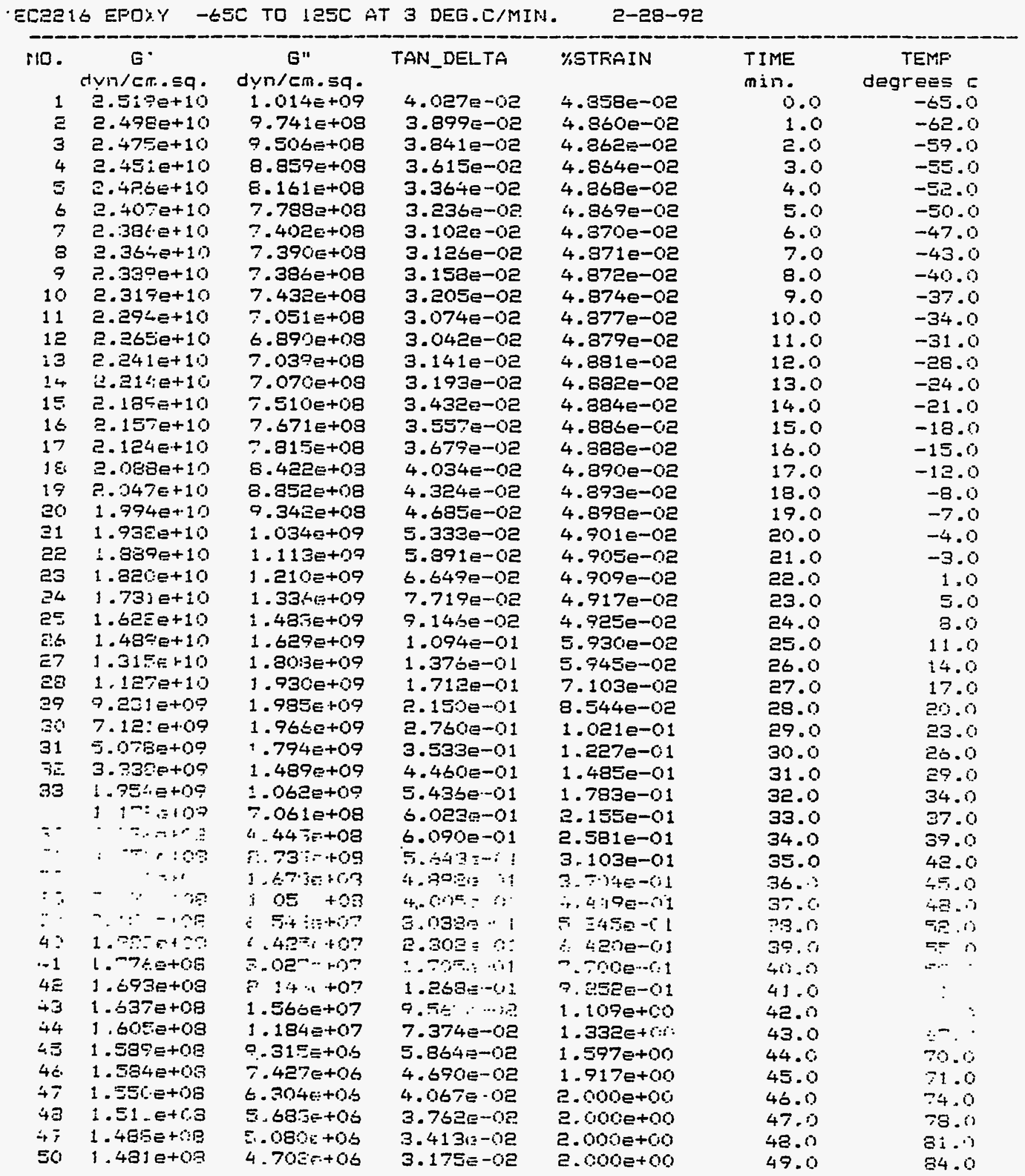




\begin{tabular}{|c|c|c|c|c|c|c|}
\hline [ ] & $\begin{array}{c}\hat{G}^{\circ} \\
\text { dvni=1 } \leq q .\end{array}$ & $\begin{array}{c}\text { G" } \\
\text { dynicn. } 59 .\end{array}$ & TArd_DEL_TA & $\because E T F A I N$ & $\begin{array}{l}\text { TIME } \\
\text { min. }\end{array}$ & $\begin{array}{c}\text { TEHF } \\
\text { degi EE: }\end{array}$ \\
\hline 51 & $1 .+8 \div E+6 \theta$ & $4.56 .5 e+06$ & $3.071 \equiv-02$ & $2.000 \equiv+00$ & 50.0 & E. .13 \\
\hline 59 & 1. J0ME+OB & $4.754=+06$ & $3.157 e-02$ & 2. 000 e+oo & 51.0 & 92.1 \\
\hline 33 & $1.505 e+09$ & $4.774 E+06$ & 3.172e-0P & $2.000=+00$ & 52.0 & $9 \leq 0$ \\
\hline 54 & i. $513 e+08$ & $4.323=+06$ & 3.178e- & $2.0000+00$ & 53.0 & 78.0 \\
\hline$=5$ & 1. $.54 . E+0 \Omega$ & $4.604=000$ & 3. 028p-02. & $2.000 e+00$ & 54.0 & 100.0 \\
\hline 52 & $1.52 c e+08$ & $4.617 E+06$ & $3.019=-02$ & $2.000 e+00$ & 55.0 & 303.0 \\
\hline 5.7 & 1. 4GBE+0A & 4. 36 $3 E+06$ & $2.975=-18$ & $2.000=+00$ & 56.0 & 105.6 \\
\hline 58 & $1.459 E+08$ & $4.321=\div 06$ & 2.7b1e-02 & $2.000 e+00$ & 57.0 & 109.6 \\
\hline 50 & $1.470 e+09$ & $4.399=+06$ & 2.74́́e-0e & $2.000 e+00$ & 58.0 & 113.0 \\
\hline sil & 1. SOEE+09 & 4. $327 E+01$ & 2.882e-02 & $2.000 e+\infty 0$ & 59.0 & 115.0 \\
\hline$\$ 1$ & $1.511 \equiv+03$ & $4.279 \equiv+06$ & 2. $833 e-02$ & E. $000 e+00$ & 60.0 & 117.0 \\
\hline$s=$ & 1. $490 e+08$ & $4.254 E+0$ S & $2.857 e-02$ & $2.000 E+00$ & 31.0 & 123.0 \\
\hline-3 & $1.43 .5 E+08$ & $4.083 e+06$ & $2.7342-02$ & $2.000 e+00$ & 62.0 & 125.0 \\
\hline$\leq 4$ & $1.473 e+00$ & $4.048 E+0 S$ & 2.747e-02 & $2.000 e+c 0$ & 63.0 & $1 \Xi 4.0$ \\
\hline
\end{tabular}


Table F-6. RMS Recorded Data for EA9309

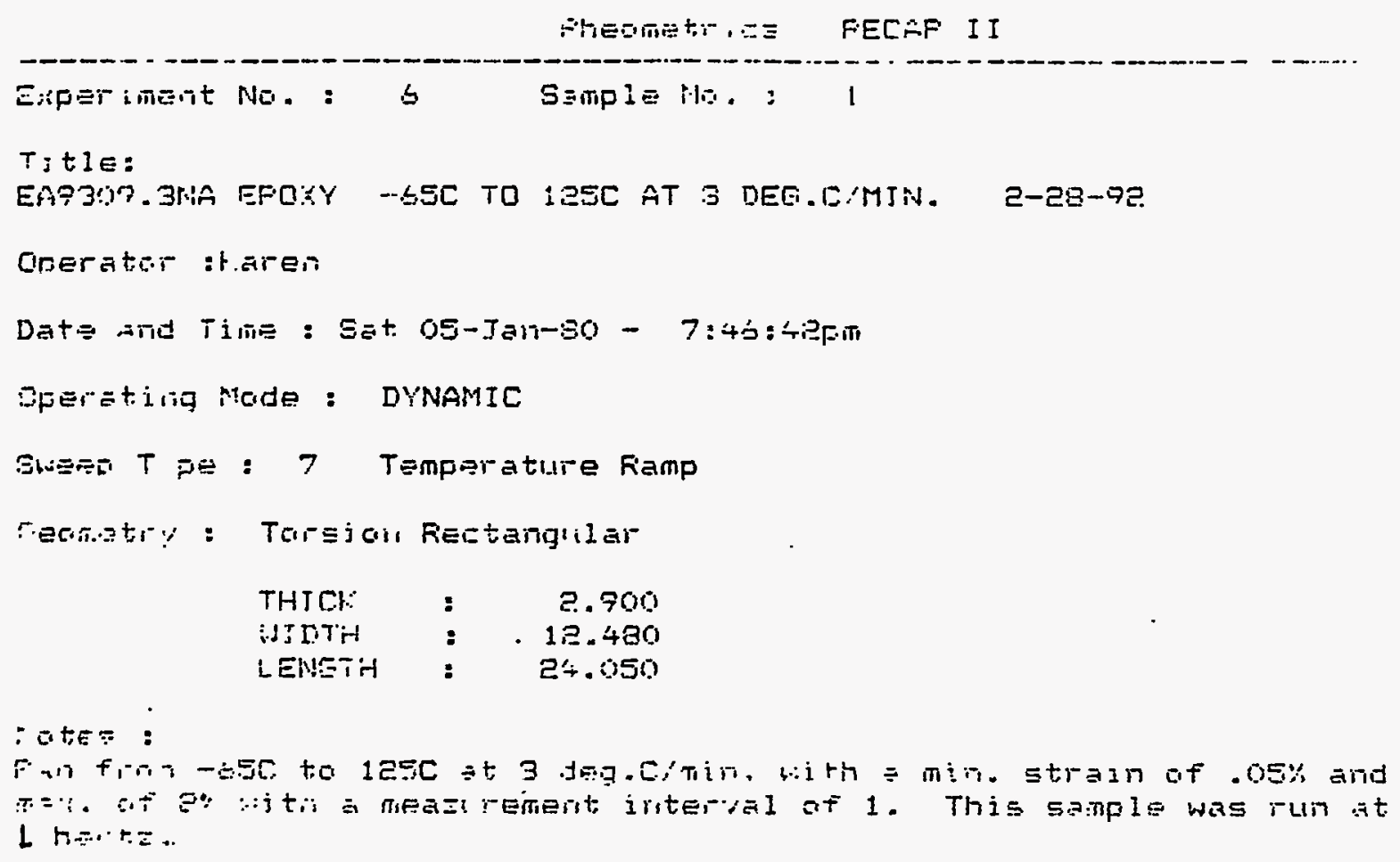




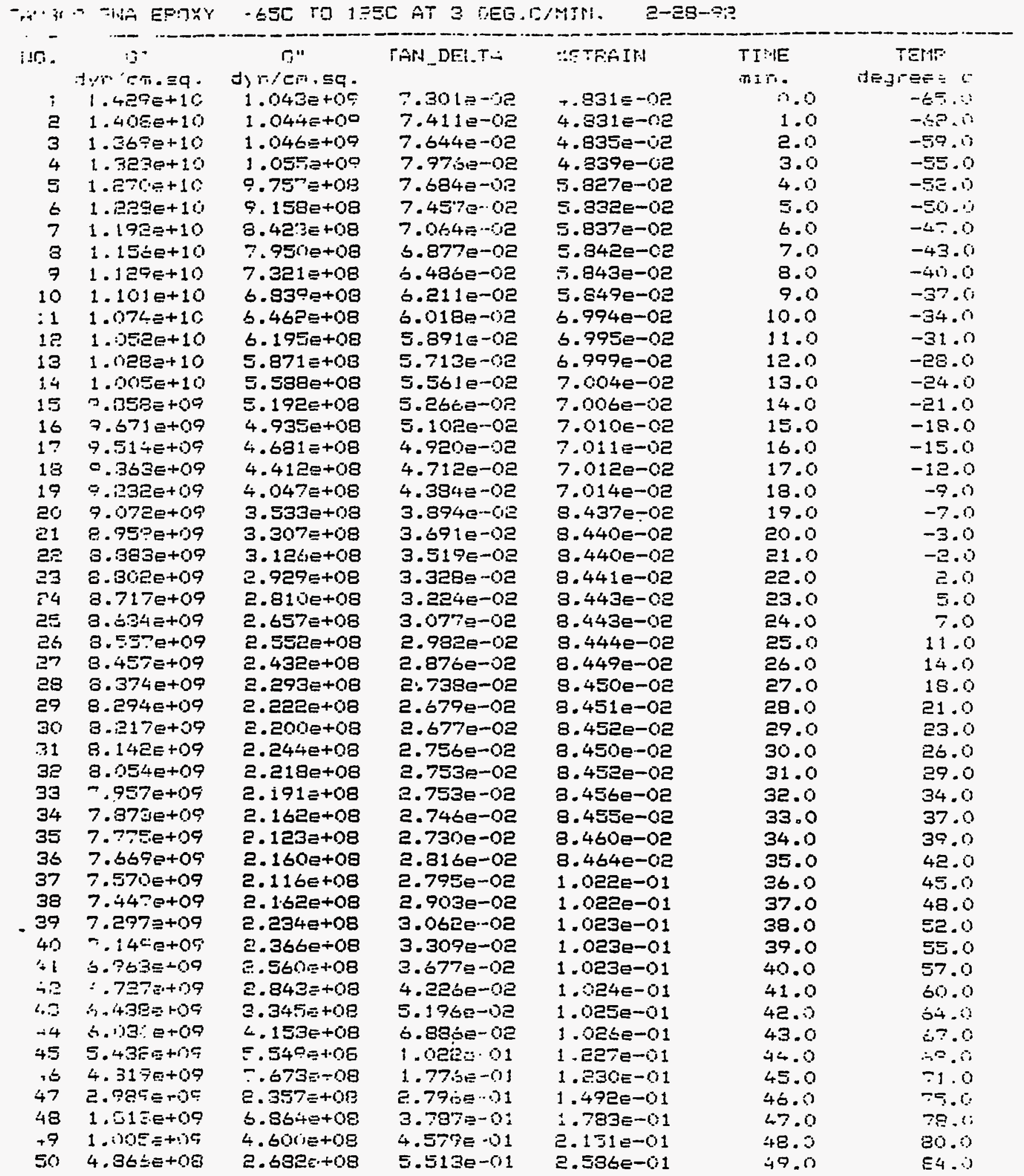




\begin{tabular}{|c|c|c|c|c|c|c|}
\hline 140. & $\begin{array}{c}G^{\circ} \\
\text { Jyn/en.sq. }\end{array}$ & $\begin{array}{c}\text { G" } \\
\text { dyn/en.sq. }\end{array}$ & TAN_DEL_TA & \%STRAIN & $\begin{array}{l}\text { TIME } \\
\text { min. }\end{array}$ & $\begin{array}{c}\text { TEMF } \\
\text { degrees }=\end{array}$ \\
\hline 51 & 2. $363 E+08$ & $1.410 e+08$ & $5.967 e-01$ & $3.097 e-01$ & 50.0 & 87.0 \\
\hline 52 & $1.454 E+08$ & $6.487 e+07$ & $4.462 E-01$ & $3.716 e-01$ & 51.0 & 92.0 \\
\hline 53 & i. $121 \mathrm{e}+08$ & $3.257 €+07$ & $2.905 \equiv-01$ & $4.455 E-01$ & 52.0 & 75.0 \\
\hline 54 & $1.001 e+08$ & $1.991 E+07$ & $1.988 e-01$ & $5.354 e-01$ & 53.0 & 93.0 \\
\hline 5.5 & $9.471=+07$ & $1.402=+07$ & $1.480 e-01$ & $b .428 e-01$ & 54.0 & 101.0 \\
\hline $5 t$ & 3. ㄷㄹㄹ 07 & $1.093 e+07$ & $1.233 e-01$ & $7.702 \mathrm{e}-01$ & 55.0 & 103.0 \\
\hline 57 & 3.866.17 & $9.4402+06$ & $1.065 e-01$ & $9.253 e-01$ & 56.0 & 105.0 \\
\hline 58 & 5. $44 Z E+07$ & $7.335 e+0 s$ & $8.689 E-02$ & $1.1100+00$ & 57.0 & 109.0 \\
\hline بك5 & 8.24:5E+07 & $5.598 e+06$ & $6.791 e-02$ & $1.331 \mathrm{e}+00$ & 58.0 & 113.0 \\
\hline so & $8.107 e+07$ & $4.279 e+06$ & $5.279 e-02$ & $1.598 e+00$ & 59.0 & 116.0 \\
\hline 31 & $8.147 E+07$ & $3.339 \mathrm{e}+06$ & $4.098 e-02$ & $1.917 e+00$ & 60.0 & 117.0 \\
\hline $6 \varepsilon$ & $9.04 \% e+07$ & $2.587 n+06$ & $3.215 e-02$ & $2.000 \mathrm{e}+00$ & 61.0 & 123.0 \\
\hline 63 & $7.764 E+07$ & $2.083 \equiv+06$ & $2.616 e-02$ & 2.000e+o0 & 62.0 & 124.0 \\
\hline b4 & $7.93 \%$ e+07 & 1. 98ZE+on & $2.497 e-02$ & $2.000 e+00$ & 63.0 & 124.0 \\
\hline
\end{tabular}


Appendix G

\section{DMA Glass Transition Temperature}




\section{DMA Glass Transition Temperature}

\section{Test Purpose:}

The purpose of this test is to look at the viscoelastic properties of optical adhesives and determine the glass transition temperature using Dynamic Mechanical Analysis (DMA).

\section{Background:}

The technique of Dynamic Mechanical Analysis is one of the most sensitive single techniques available for characterizing and interpreting the mechanical behavior of materials. DMA is based on observing the viscoelastic response of materials subjected to a small oscillatory strain. The DuPont 983 DMA uses a flexural bending deformation mode of strain, but also has the capability for other deformations such as shear.

The DMA technique separates the viscoelasticity of a material into the two components of Modulus (E): a real part, which is the elastic modulus (E'); and an imaginary part, which is the damping or viscous component (E"). The standard complex variable notation is: $E=E^{\prime}+i E^{\prime \prime}$. This separation of measurements into the two components describing two independent processes within the materials' elasticity (energy storage) and viscosity (energy dissipation) is the fundamental characteristic of dynamic mechanical analysis that separates it from other mechanical testing techniques.

The high sensitivity of DMA is particularly advantageous in the measurement and interpretation of transitions that are studied by the measurement of tan delta (the ratio of lost to stored energy). In the Resonant Mode, the 983 DMA operates on the mechanical principle of forced resonant vibratory motion at a fixed amplitude (strain), which is selected by the operator. The arms and sample are displaced by the electromagnetic driver, which subjects the sample to a fixed deformation and sets the system in resonant oscillation. The amplitude and frequency of oscillation are measured by the LVDT. That measurement, together with the amount of energy sent to the driver, is used to calculate the desired viscoelastic properties.

\section{Sample Preparation:}

Samples, approximately $0.5^{\prime \prime} \times 0.125^{\prime \prime} \times 1.5^{\prime \prime}$ were made and tested from each adhesive. The samples were mixed and cured per Table A-1 in Appendix A. 


\section{Test Procedure:}

The DMA tests were performed using a TA instruments model 983 dynamic mechanical analyzer. The DMA was run in the resonant mode at a heat rate of $3^{\circ} \mathrm{C}$ per minute from $-65^{\circ} \mathrm{C}$ to $80^{\circ} \mathrm{C}$.

\section{Test Results:}

Table G-1 shows the glass transition temperature for the different optical adhesives.

Figures $\mathrm{G}-1$ through $\mathrm{G}-5$ show the frequency response versus temperature.

Table G-1: Glass Transition Temperature by DMA:

\begin{tabular}{||l|c|c||}
\hline \hline \multicolumn{1}{|c|}{ Material } & \multicolumn{2}{|c|}{ Glass Transition Temperature } \\
\hline \hline & $\left({ }^{\circ} \mathrm{F}\right)$ & $\left({ }^{\circ} \mathrm{C}\right)$ \\
\hline A12 1:1 & 130.5 & 54.7 \\
\hline A12 1:2 & 74.5 & 23.6 \\
\hline A12 2:3 & 85.8 & 29.9 \\
\hline EC 2216 (gray) & 69.4 & 20.8 \\
\hline EA 9309.3 NA & 119.0 & 66.1 \\
\hline
\end{tabular}


Sample: A12 1: 1

Size : $34.73 \times 12.40 \times 2.80 \mathrm{~mm}$

Method : LNCA SUBAMBIENT FILLED

Comment: DMA RESONANT 3 DEGREES C/MINUTE
F11日 : C: RES.03

Operator: DAVID A. SPIEKER

Run Date: 12-May-92 08: 32

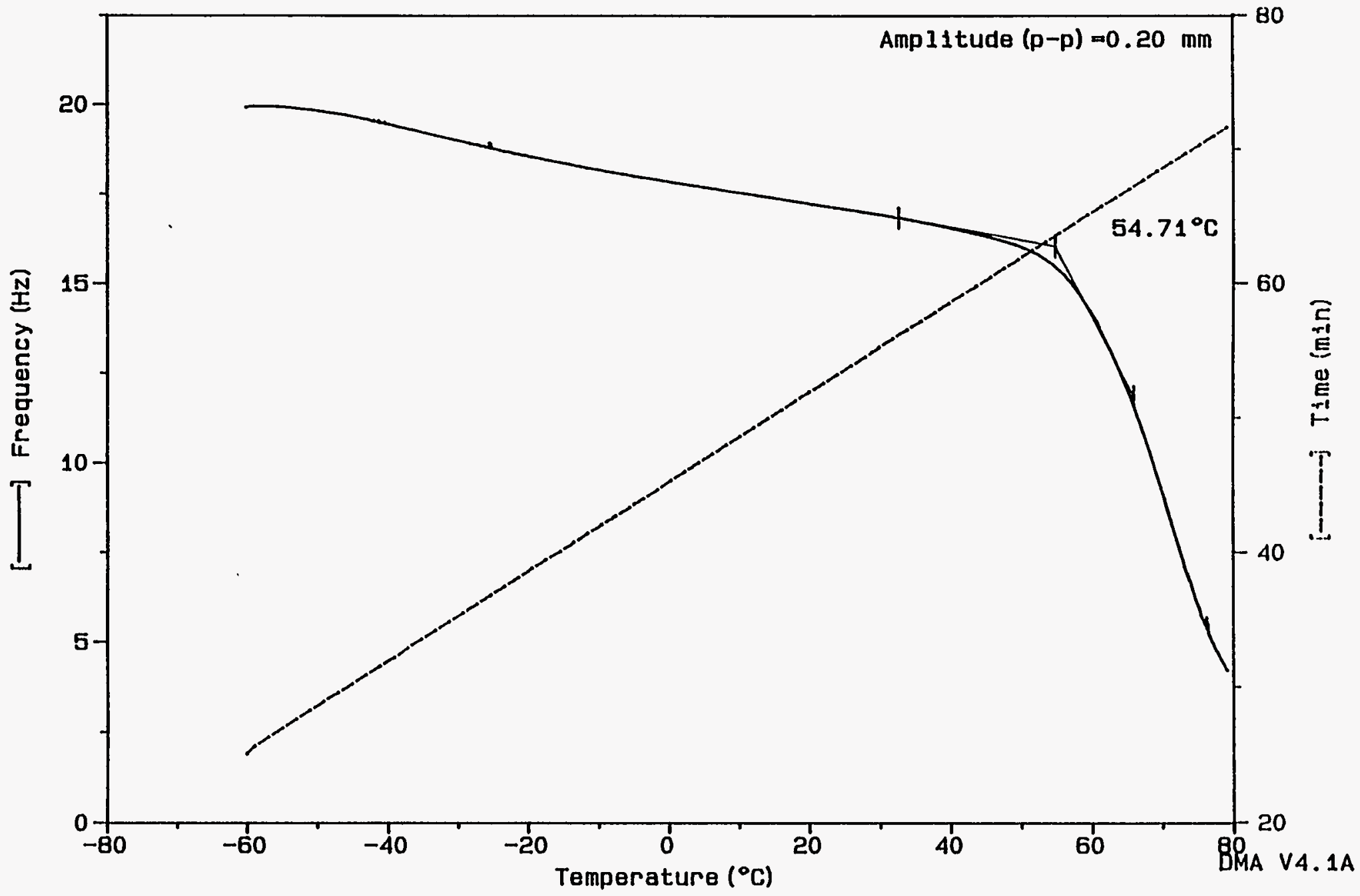

Figure G-1. Frequency Response as a Function of Temperature for A12 1:1 Ratio 
Sample: A12 1: 2

Size: $34.73 \times 12.40 \times 2.80 \mathrm{~mm}$

Method : LNCA SUBAMBIENT FILLED

Comment: DMA RESONANT 3 DEGREES C/MINUTE

F110 : C: AES.02

Operator: DAVID A. SPIEKER

Run Date: 11-May-92 13: 57

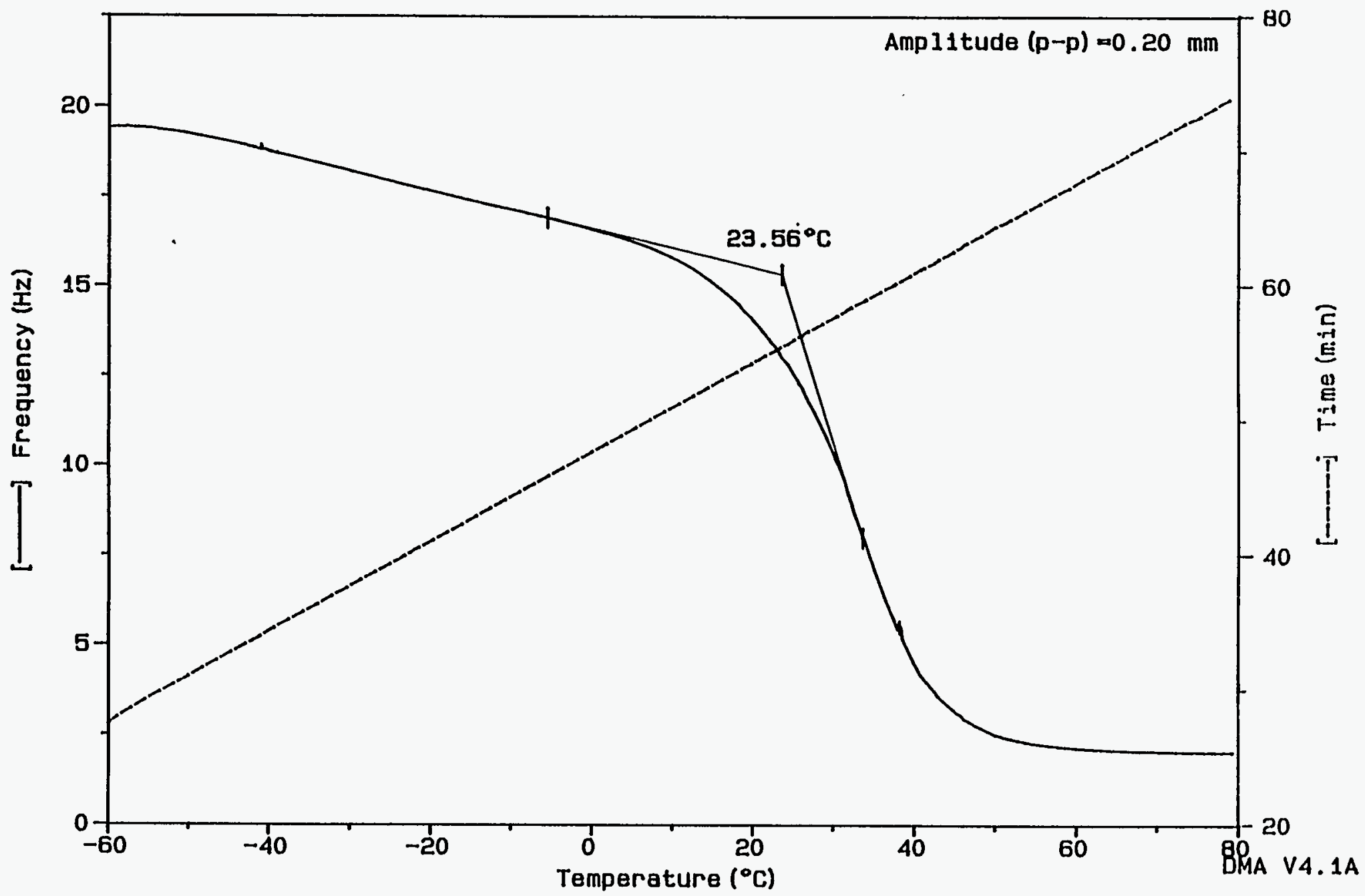

Figure G-2. Frequency Response as a Function of Temperature for A12 1:2 Ratio 

Sample : A12 2: 3
Size : $34.73 \times 12.40 \times 2.80 \mathrm{~mm}$
Method : LNCA SUBAMBIENT FILLED
Comment: DMA RESONANT 3 DEGREES C/MINUTE
File : C: RES.04
Operator: DAVID A. SPIEKEA
Aun Date: 12-May-92 09: 59

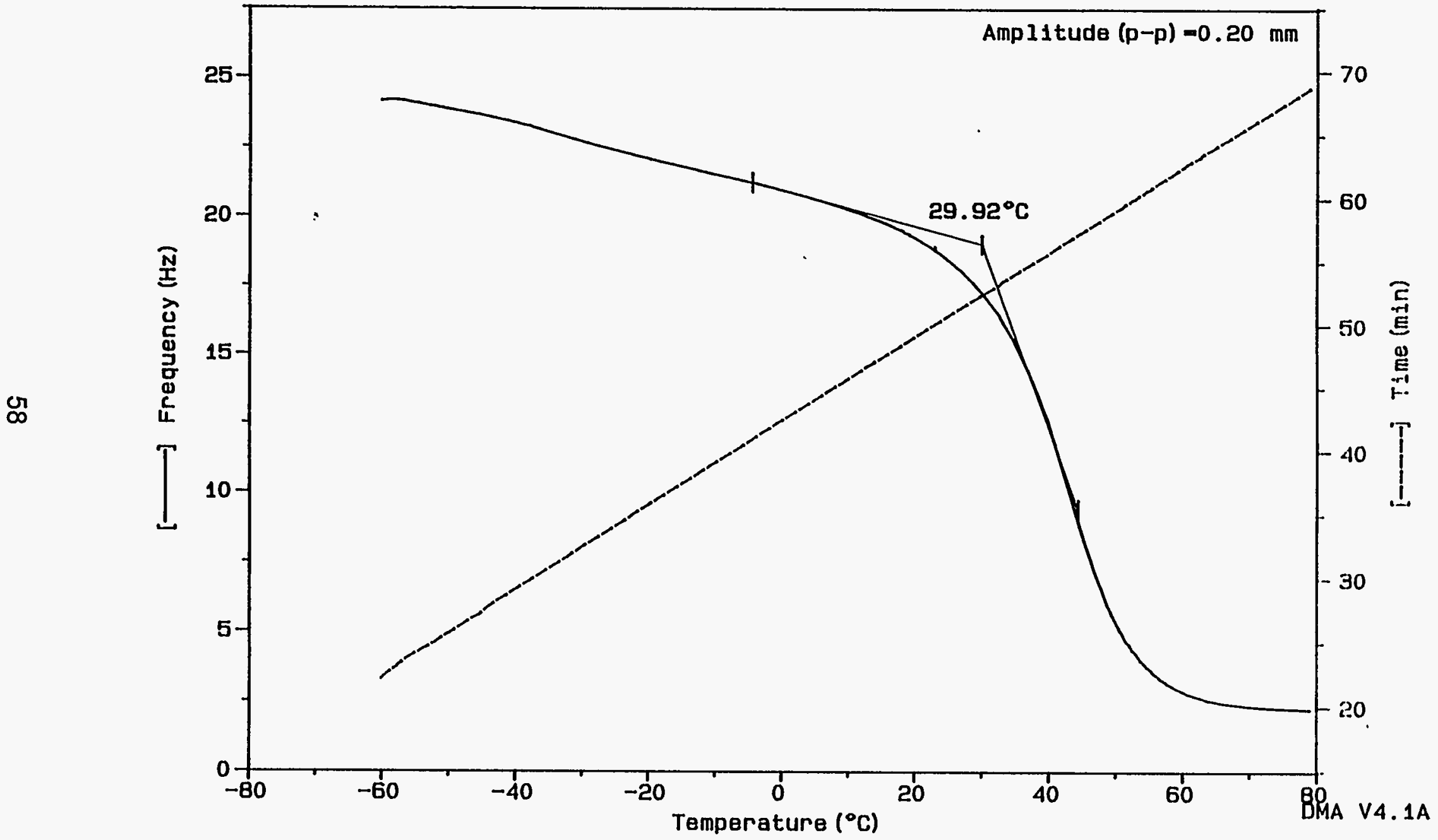

Figure G-3. Frequency Response as a Function of Temperature for A12 2:3 Ratio 

Sample : EC2216
S1ze: $34.73 \times 12.40 \times 2.80 \mathrm{~mm}$
Method : LNCA SUBAMBIENT FILLED
Comment: DMA RESONANT 3 DEGREES C/MINUTE
File : C: RES.05
Operator: DAVID A. SPIEKER
Run Date: 12-May-92 11: 31

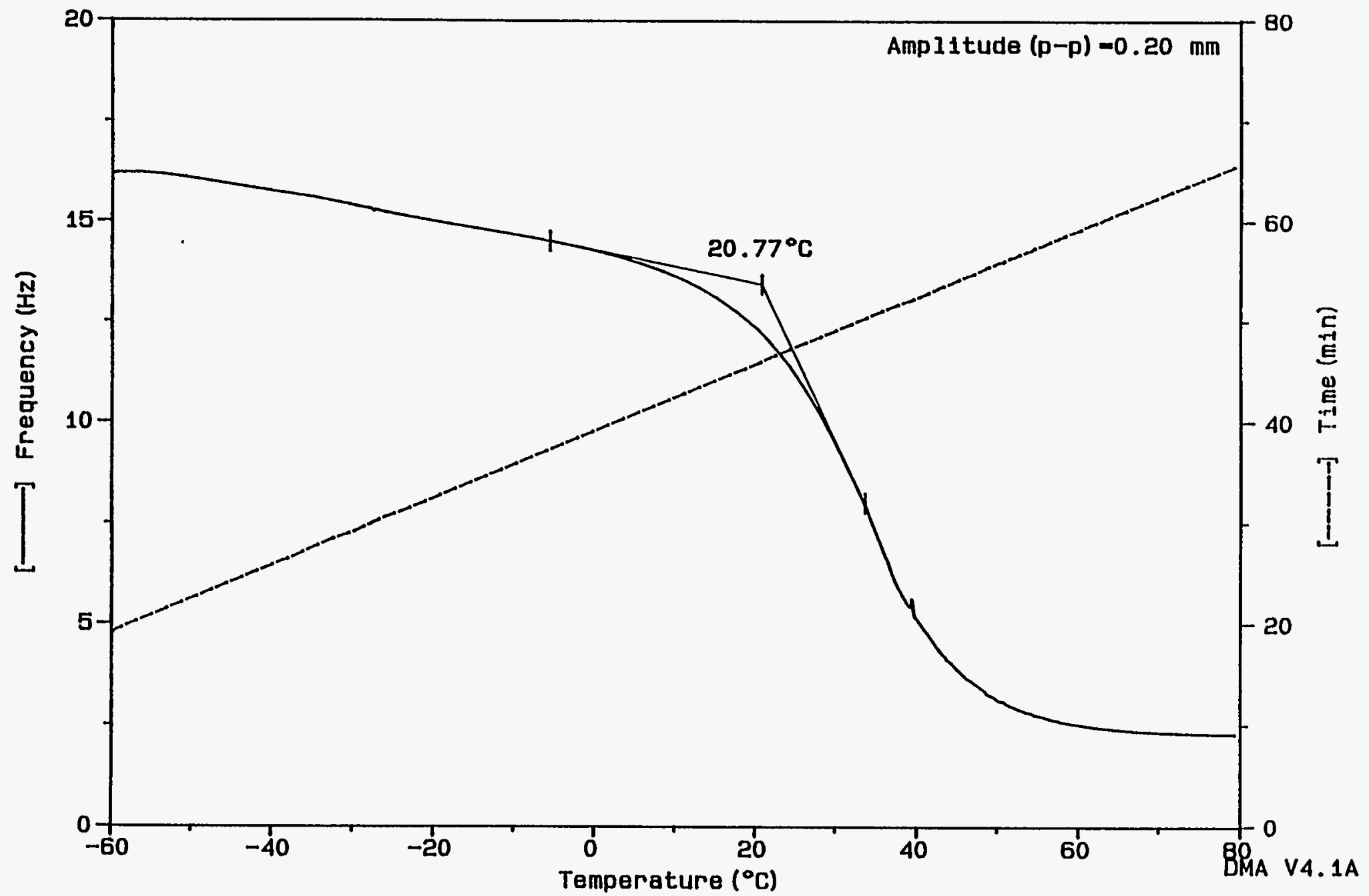

Figure G-4. Frequency Response as a Function of Temperature for EC2216 
Sample : EA 9309.3NA

Size: $34.73 \times 12.40 \times 2.80 \mathrm{~mm}$

Method : LNCA SUBAMBIENT FILLED

Comment: DMA RESONANT 3 DEGREES C/MINUTE

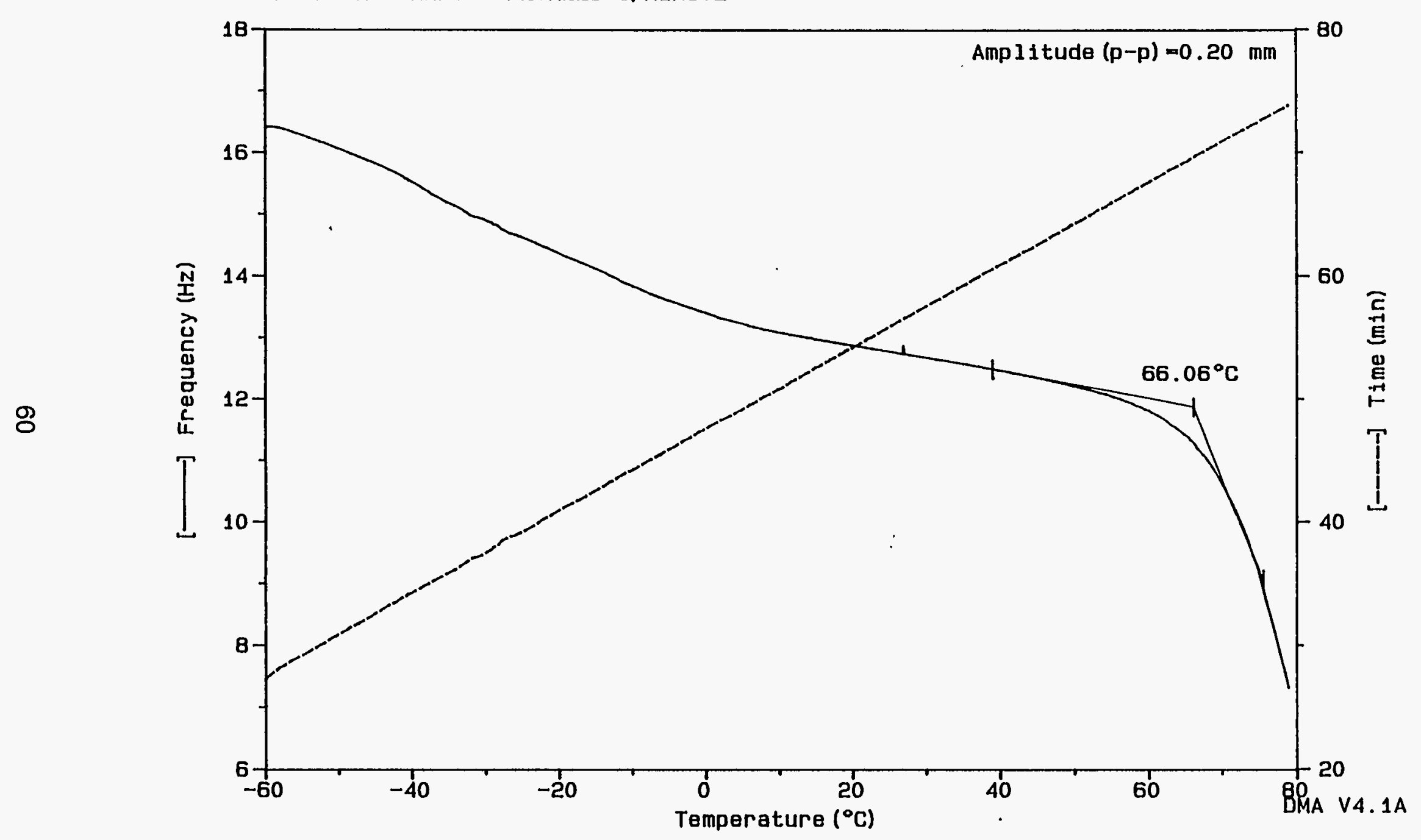

File : C: RES.01

Operator: DAVID A. SPIEKER

Run Date: 11-May-92 11: 18

Figure G-5. Frequency Response as a Function of Temperature for EA9309.3 NA 
Appendix $\mathrm{H}$

\section{Thermal Stability}




\section{Thermal Stability}

\section{Test Purpose:}

This test will determine the thermal stability of the optical adhesives using Thermogravimetric Analysis.

\section{Background:}

Thermogravimetric Analysis (TGA) is an analytical technique which measures weight loss of a material versus temperature at a programmed heating rate. TGA analysis can provide information on organic materials such as thermal stability, decomposition temperature, decomposition kinetics, filler contents and oxidation degradation studies.

The TGA apparatus is a Du Pont 9900 controller with 951 thermogravimetric analyzer which is essentially a very sensitive thermal balance. The sample pan serves as the weight tray and is surrounded with a quartz tube and inserted into a furnace chamber. The furnace heats the sample up to $1000^{\circ} \mathrm{C}$.

\section{Sample Preparation:}

One sample (of approximately $20 \mathrm{mg}$ ) was made and tested from each adhesive per Table A-1 in Appendix A.

\section{Test Procedure:}

The samples were tested in air at 10 degrees per minute from room temperature to $700^{\circ} \mathrm{C}$. The weight was monitored and recorded. The temperature was noted when the material significantly started outgassing and losing weight. The residue was weighed at the end of the test.

\section{Test Results:}

Table $\mathrm{H}-1$ shows the thermal decomposition temperature and the residue (percent filler). The test data are included as Figures $\mathrm{H}-1$ through $\mathrm{H}-5$. The figures note the sample material, initial weight, and rate the test is performed and then plots the percent weight versus the temperature. The thermal decomposition temperature is based on the intersection of lines extended from the $100 \%$ line and a line representative of the slope during decomposition. As can be seen from the figures, the material actually begins decomposing at a lower temperature. 
Table H-1: Thermal Decomposition Temperature and Residue:

\begin{tabular}{||l|c|c|c||}
\hline Material & \multicolumn{2}{|l||}{$\begin{array}{l}\text { Thermal Decomposition } \\
\text { Temperature }\end{array}$} & $\begin{array}{l}\text { Residue } \\
\text { (Percent filler) }\end{array}$ \\
\hline \hline & $\left({ }^{\circ} \mathrm{F}\right)$ & $\left.{ }^{\circ} \mathrm{C}\right)$ & \\
\hline A12 1:1 & 698.2 & 370.1 & 29.8 \\
\hline A12 1:2 & 683.6 & 362.0 & 30.6 \\
\hline A12 2:3 & 677.3 & 358.5 & 34.5 \\
\hline EC 2216 (gray) & 651.9 & 344.4 & 30.3 \\
\hline EA 9309.3 NA & 604.4 & 318.0 & 7.6 \\
\hline
\end{tabular}




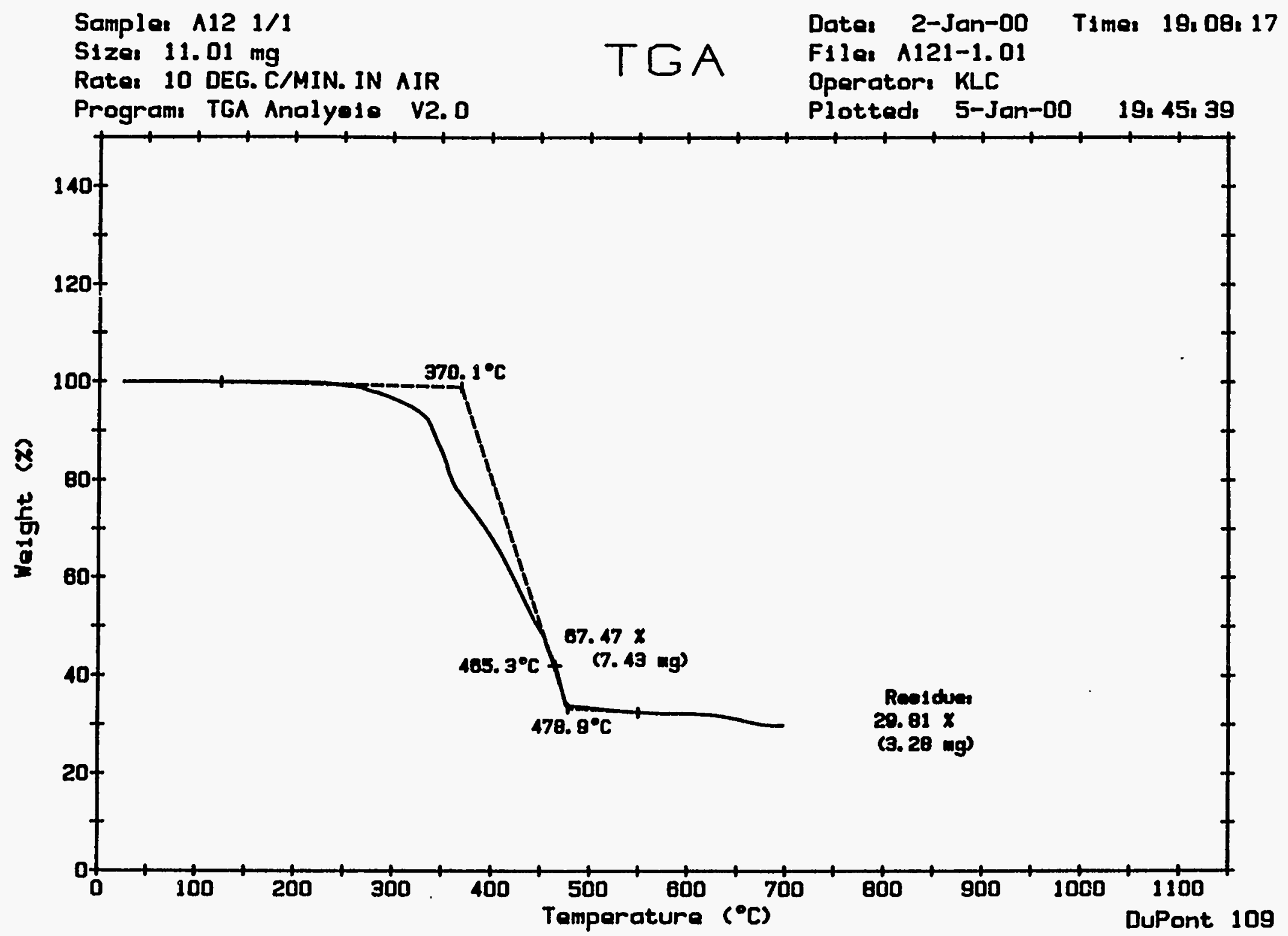

Figure H-1. Percent Weight as a Function of Temperature for A12 1:1 Ratio 


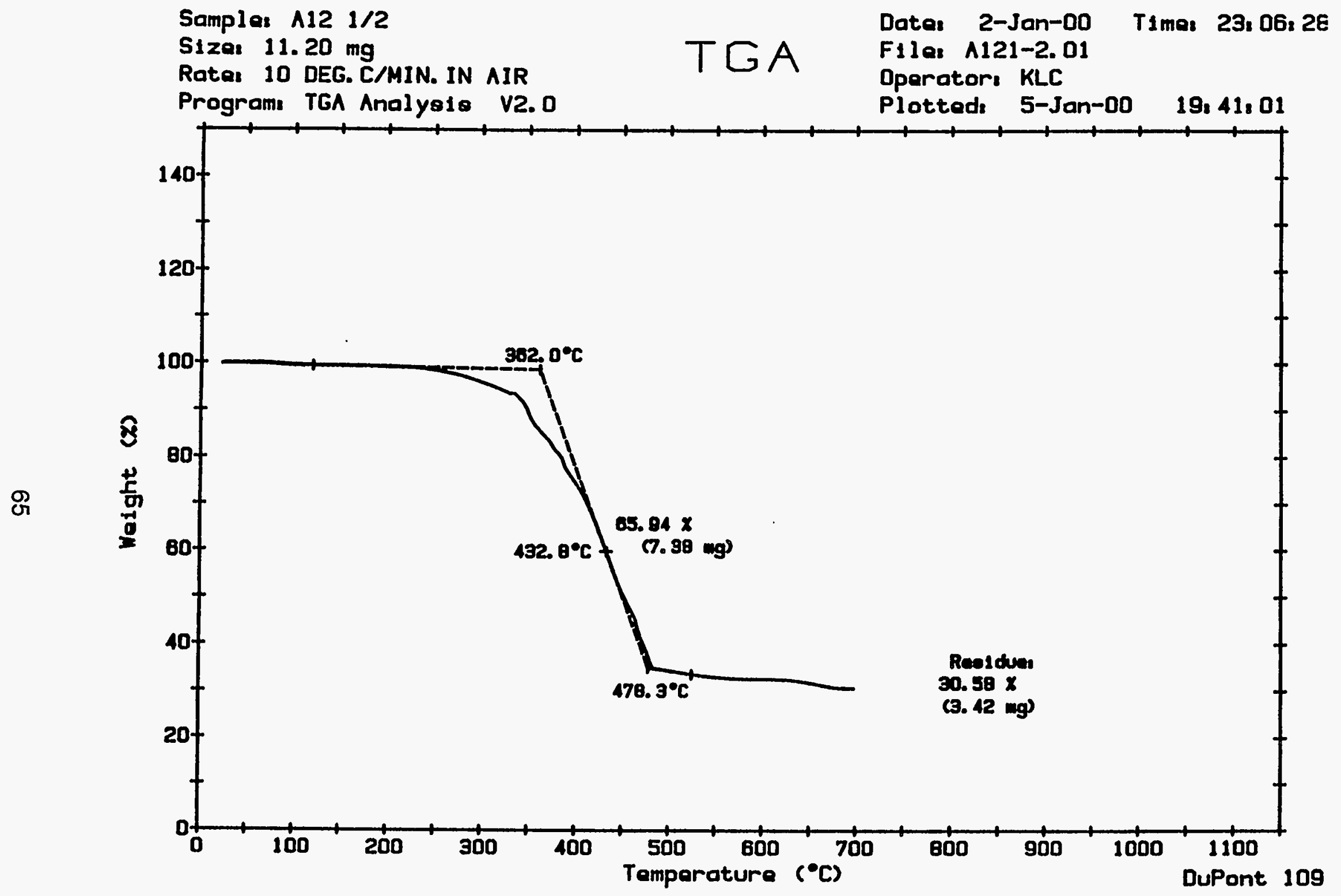

Figure H-2. Percent Weight as a Function of Temperature for A12 1:2 Ratio 


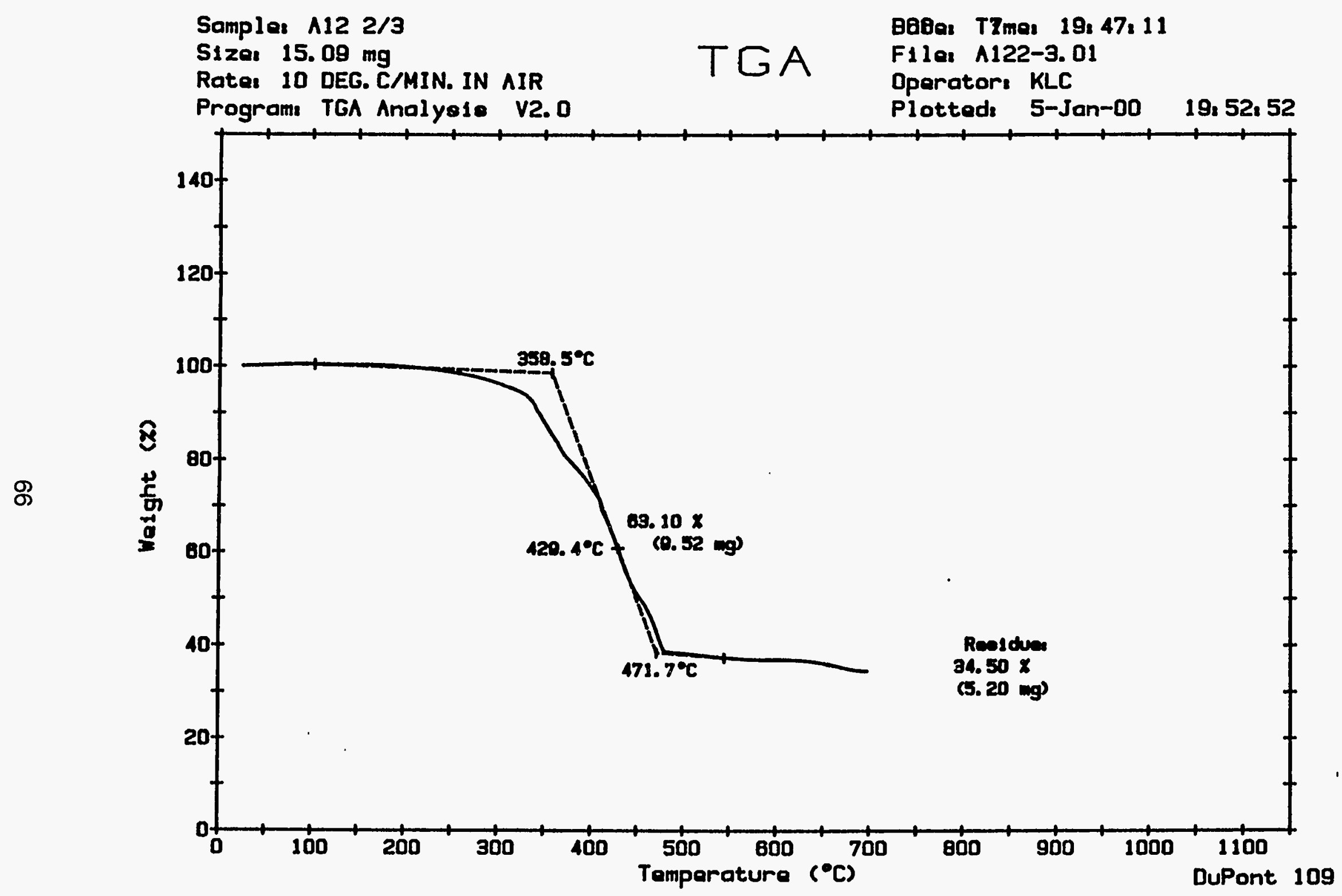

Figure H-3. Percent Weight as a Function of Temperature for A12 2:3 Ratio 


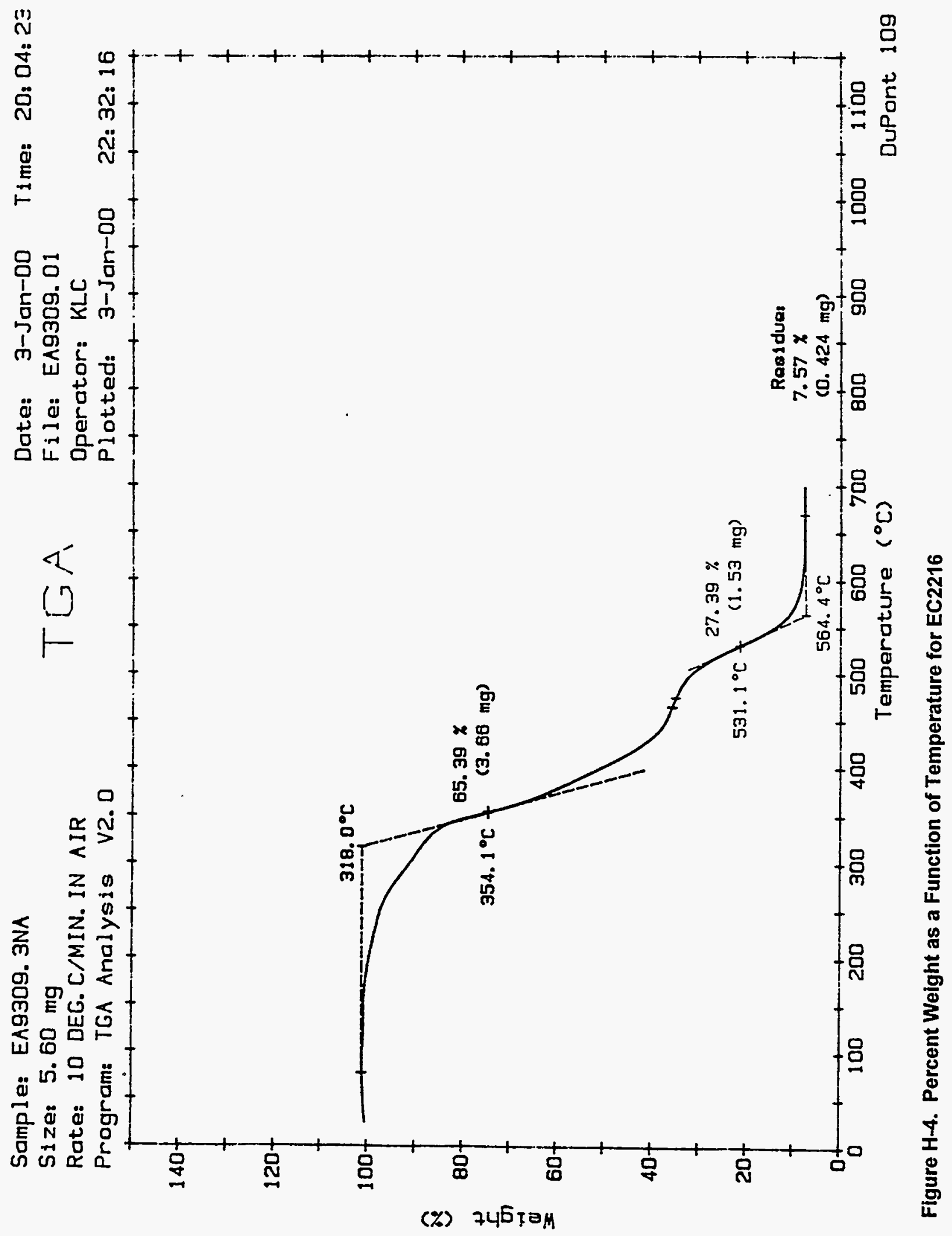




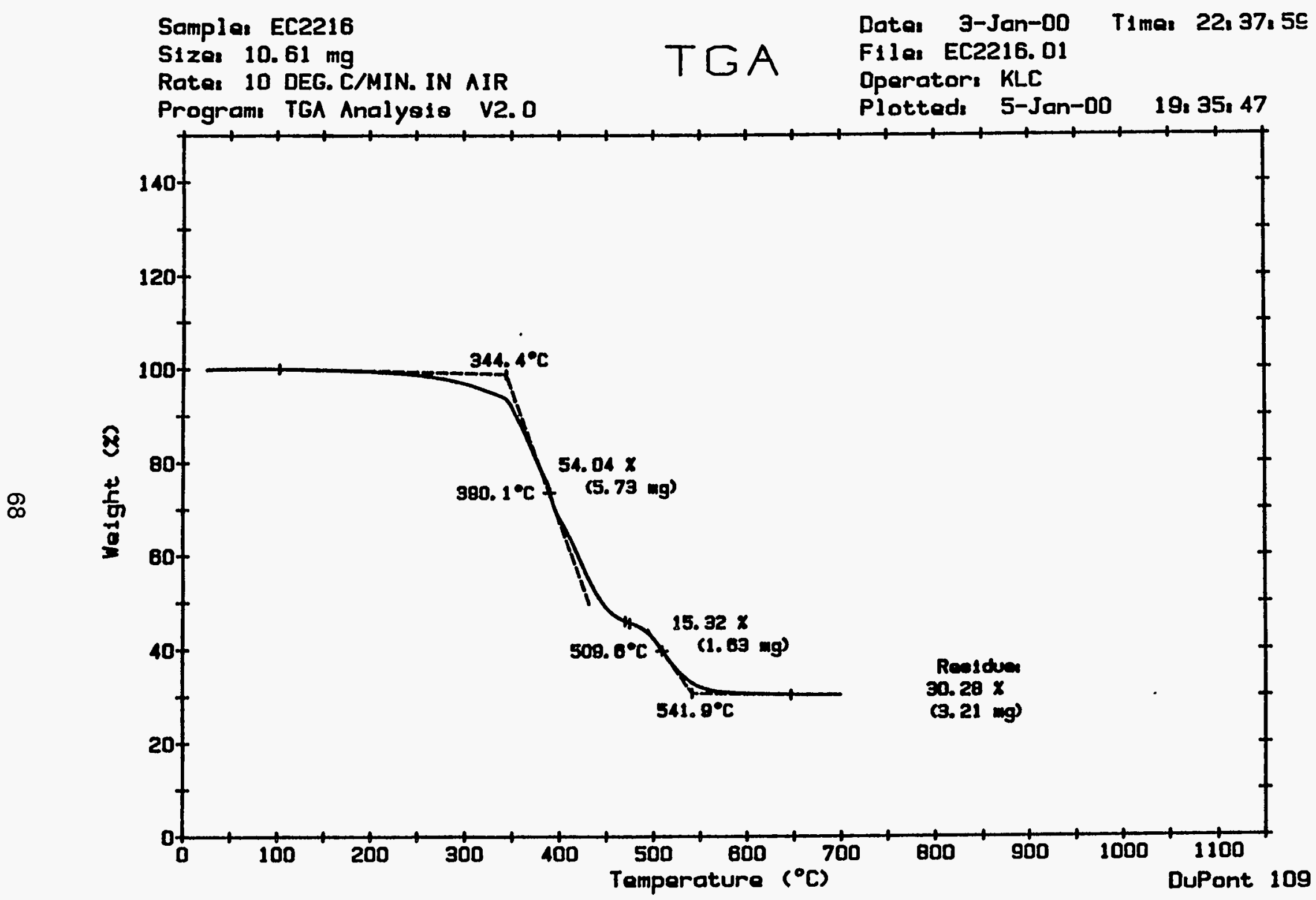

Figure H-5. Percent Weight as a Function of Temperature for EA9309.3 NA 
Appendix I

Specific Heat 


\section{Specific Heat}

\section{Test Purpose:}

The purpose of this test is to look at the specific heat as a function of temperature and also give the kinetics of cure using Differential Scanning Calorimetry (DSC).

\section{Background:}

The technique of Differential Scanning Calorimetry (DSC) measures heat flow into (endothermic reaction) or out of (exothermic reaction) a sample as the sample undergoes a change of state, chemical reaction, decomposition or various second order transitions. The temperature at which their changes occur can be accurately measured as well as a quantitative determination of the energy associated with any of their transitions. The DSC can be used to determine specific heat of materials, heat of fusion, heat of reaction, reaction kinetics, and activation energy (reaction) of organic materials.

The DSC apparatus is a DuPont 9900 controller with 912 DSC dual cell and cell base. The DSC accurately measures the temperature at which chemical reactions or transitions occur as well as being able to quantitate the energy associated with reactions or transitions. Samples and an inert reference material are placed on raised sample and reference platforms of a constanton dish, which transfers heat to both sample and reference. Sample temperature is monitored by a chromel-alumel junction on the sample disk. Differential temperature between sample and reference is monitored by chromel-constanton junction formed by the constanton disk and chromel wafer that is in contact with the underside of each raised platform. The calorimetric sensitivity and temperature accuracy are calibrated using metals of known heat of fusion to a sensitivity on $0.01 \mathrm{~mW} / \mathrm{cm}$.

\section{Sample Preparation:}

Samples were mixed as indicated in Table I-1 and then cured as indicated in the next section. 
Table I-1: Adhesive Mix Ratios

\begin{tabular}{||l|c|c||}
\hline \multirow{2}{*}{ Adhesive } & \multicolumn{2}{|c|}{ Mix Ratio* $^{*}$} \\
\cline { 2 - 3 } & A Comp. & B Comp. \\
\hline \hline A12 1:1 & 1 & 1 \\
\hline A12 1:2 & 1 & 2 \\
\hline A12 2:3 & 2 & 3 \\
\hline EC2216 (Gray) & 1.4 & 1 \\
\hline EA9309.3NA & 10 & 2.2 \\
\hline
\end{tabular}

* Material was mixed based on weight, i.e., 10:2.2 would be mixed using 10 grams of A component mixed with 2.2 grams of B component or some factor thereof.

\section{Test Procedure:}

Kinetics tests were performed using a TA instruments differential scanning calorimeter. The ASTM E 698 analysis program was used for the runs at the five different heat rates. The average room temperature was $22^{\circ} \mathrm{C}$. The samples were cured as follows:

A12:

Five samples, one at each heat rate was cured at $2,4,6,8$, and $10 \mathrm{deg} . \mathrm{C} / \mathrm{min}$. for each mix ratio.

One sample was tested after setting at room temperature for 24 hours. Two samples were cured for two hours at $160^{\circ} \mathrm{F}$ after the 24 hours at room temperature.

One sample was tested after curing for 7 days at room temperature. EC2216:

Five samples, one at each heat rate, were cured at $2,4,6,8$, and $10 \mathrm{deg} . \mathrm{C} / \mathrm{min}$.

One sample was tested after setting at room temperature for 16 hours. Two samples were cured for two hours at $160^{\circ} \mathrm{F}$ after the 16 hours at room temperature.

One sample was tested after curing for 7 days at room temperature.

\section{EA9309.3NA:}

Five samples, one at each heat rate, were cured at $2,4,6,8$, and $10 \mathrm{deg} . \mathrm{C} / \mathrm{min}$.

One sample was tested after setting at room temperature for 24 hours.

Three samples were cured for 7 days at room temperature. 


\section{Test Results:}

Figure $\mathrm{I}-1$ shows a set of curves for all the optical adhesives of specific heat as a function of temperature from $-55^{\circ} \mathrm{C}$ to $75^{\circ} \mathrm{C}$.

DSC plots at the various heat rates $\left(2,4,6,8\right.$, and $\left.10^{\circ} \mathrm{C}\right)$ are shown for the EC 2216 in Figures I-2 through I-6. The area under the curve is the Heat of Reaction. The temperature noted at the top of the curve is the temperature at which fifty percent of the reaction has occurred. Figure 1-7 shows a plot of the Log of the Heat rate for the EC 2216 versus reciprocal of the temperature for the five different heat rates. This is essentially a data verification check to assure no first order errors. The slope of the curve is the activation energy and the $Y$ intercept is the Frequency Factor $(Z)$. The frequency factor gives the frequency of reactions as a function of temperature.

Figure $1-8$ shows the predicted cure rate at $71^{\circ} \mathrm{C}$ and room temperature $\left(22^{\circ} \mathrm{C}\right)$ for the EC 2216. Eact is the Activation Energy, $\log (Z)$ is the Frequency Factor and Enthalpy is the Heat of Reaction based on the average of five runs. The next curve in Figure 1-9 shows the predicted cure rate at room temperature and the actual measured value after 24 hours for the EC 2216. The other adhesives had similar shaped curves. Table I-2 shows a summary of the Heat of Reaction, Activation Energy, and Frequency Factor for all the different optical adhesives

Table I-2: Heat of Reaction, Activation Energy, Frequency Factor

\begin{tabular}{|l|c|c|c||}
\hline Material & $\begin{array}{c}\text { Heat of Reaction } \\
\text { (J/gm.) }\end{array}$ & $\begin{array}{c}\text { Activation } \\
\text { Energy (KJ/mole) }\end{array}$ & $\begin{array}{c}\text { Frequency } \\
\text { Factor (1/min.) }\end{array}$ \\
\hline \hline A12 1:1 & 204.8 & 57.4 & 7.372 \\
\hline A12 2:3 & 140.9 & 55.9 & 7.251 \\
\hline A12 1:2 & 127.9 & 51.5 & 6.569 \\
\hline EC 2216 (gray) & 164.6 & 51.1 & 6.617 \\
\hline EA 9309.3 NA & 265.7 & 48.6 & 6.346 \\
\hline
\end{tabular}



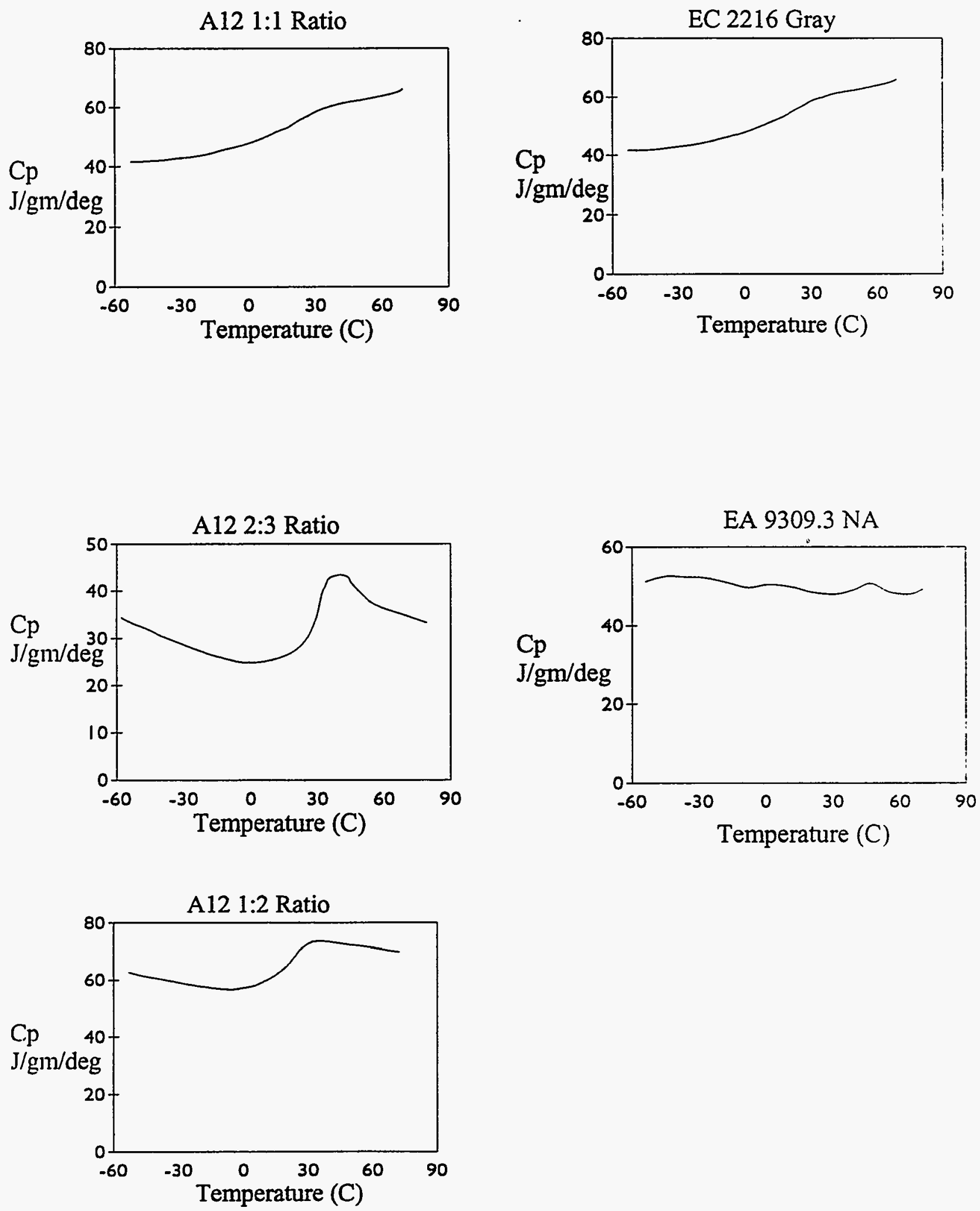

Figure 1-1. Specific Heat as a Function of Temperature for the Optical Adhesives 


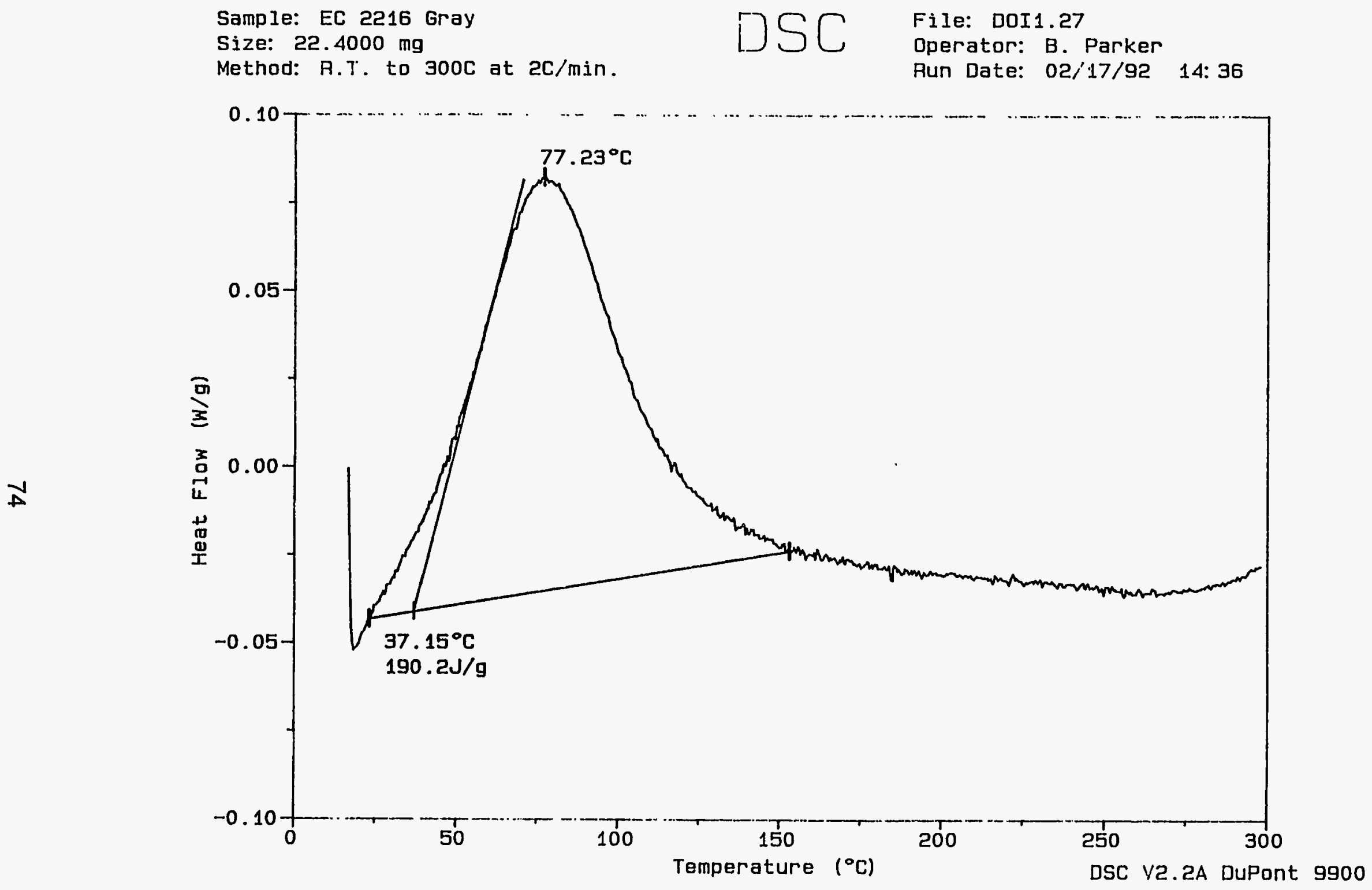

Figure l-2. Heat Flow as a Function of Temperature at $2^{\circ} \mathrm{C} / \mathrm{min}$ for $\mathrm{EC2216}$ 


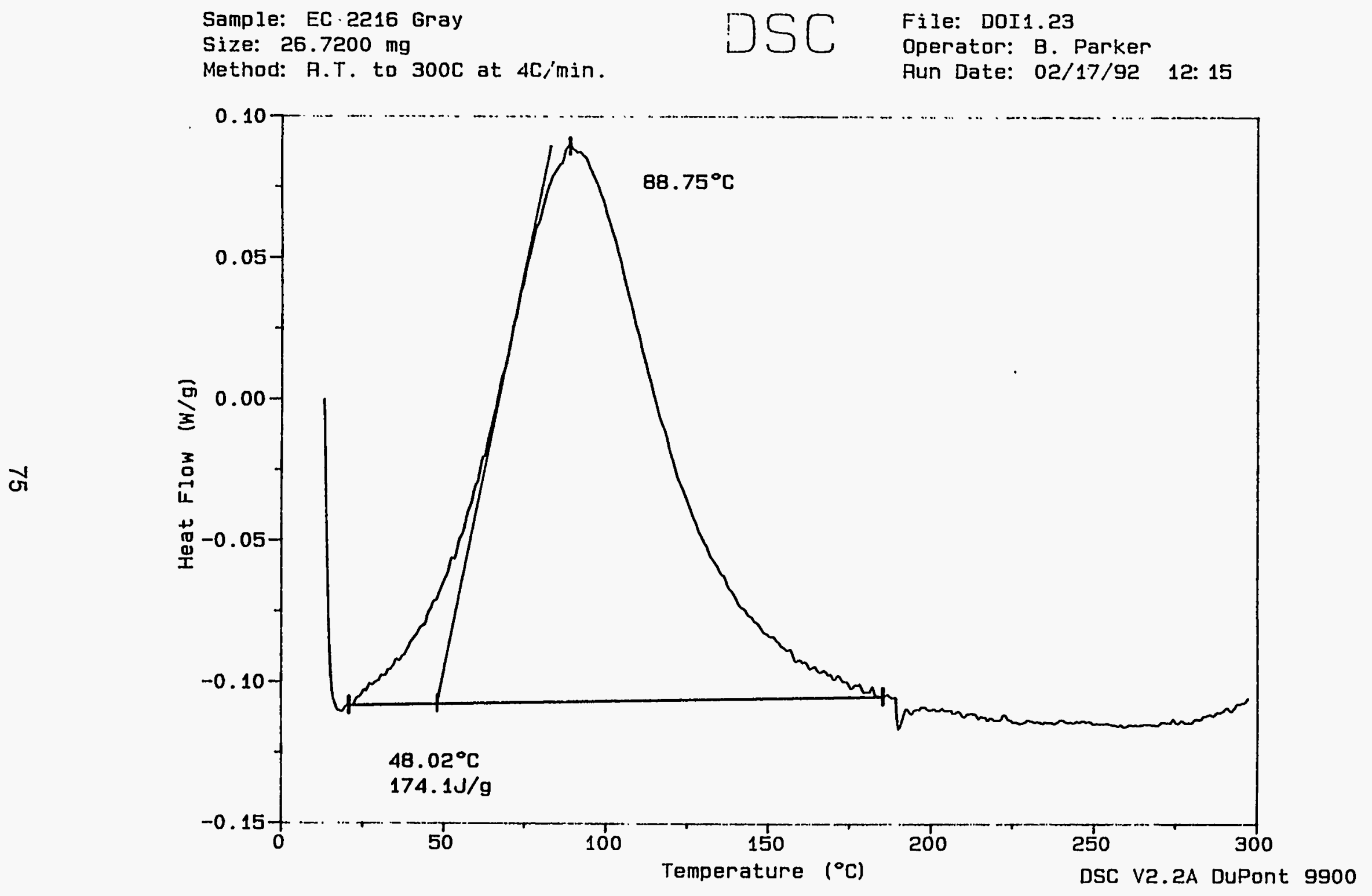

Figure 1-3. Heat Flow as a Function of Temperature at $4^{\circ} \mathrm{C} / \mathrm{min}$ for EC2216 


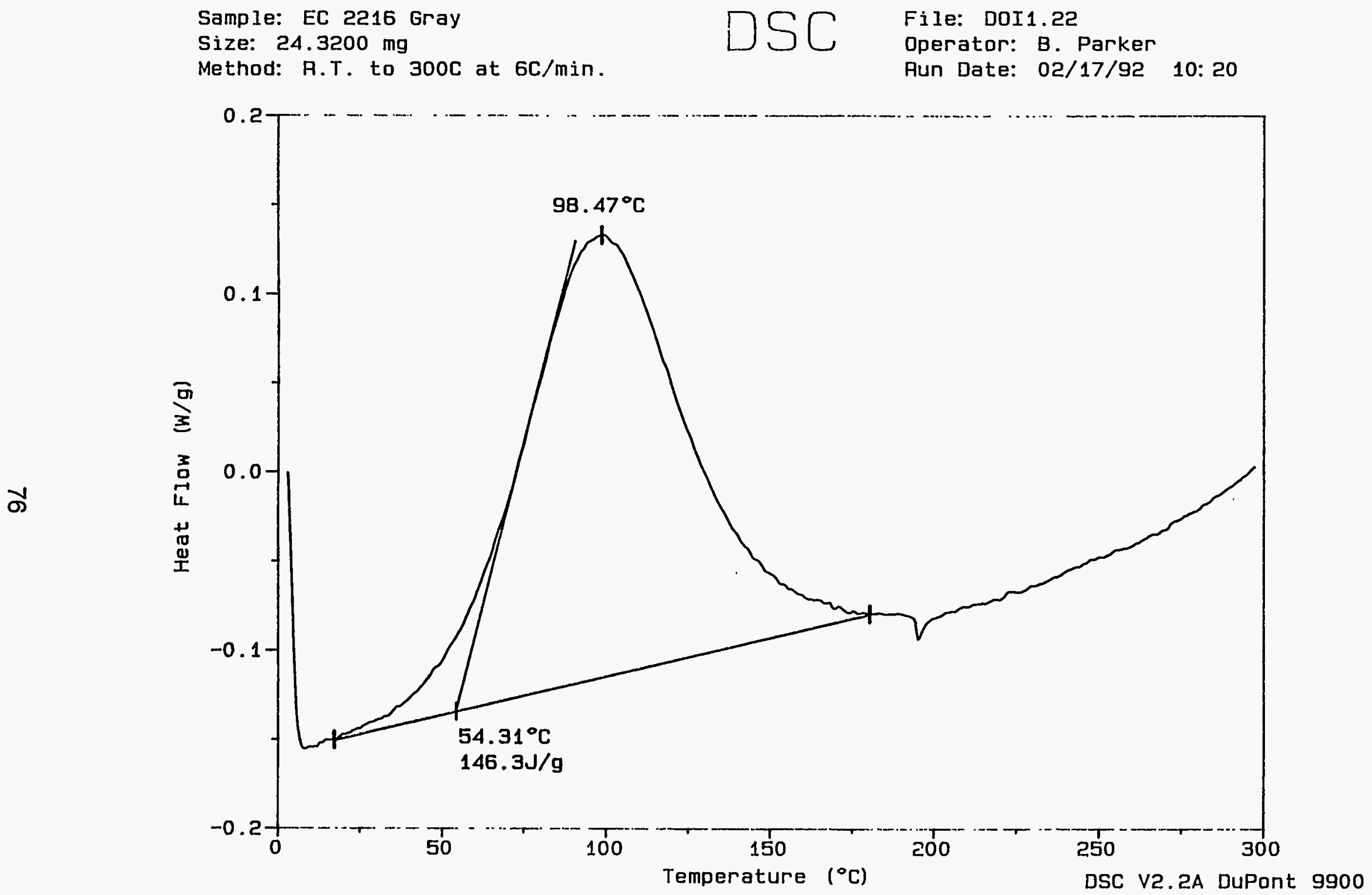

Figure 1-4. Heat Flow as a Function of Temperature at $6^{\circ} \mathrm{C} / \mathrm{min}$ for EC2216 
Sample: FC 2216 Gray

Size: $26.4300 \mathrm{mg}$

Method: A.T. to $300 \mathrm{C}$ at $\mathrm{BC} / \mathrm{min}$.

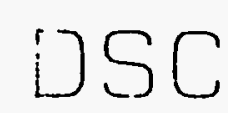

DSC

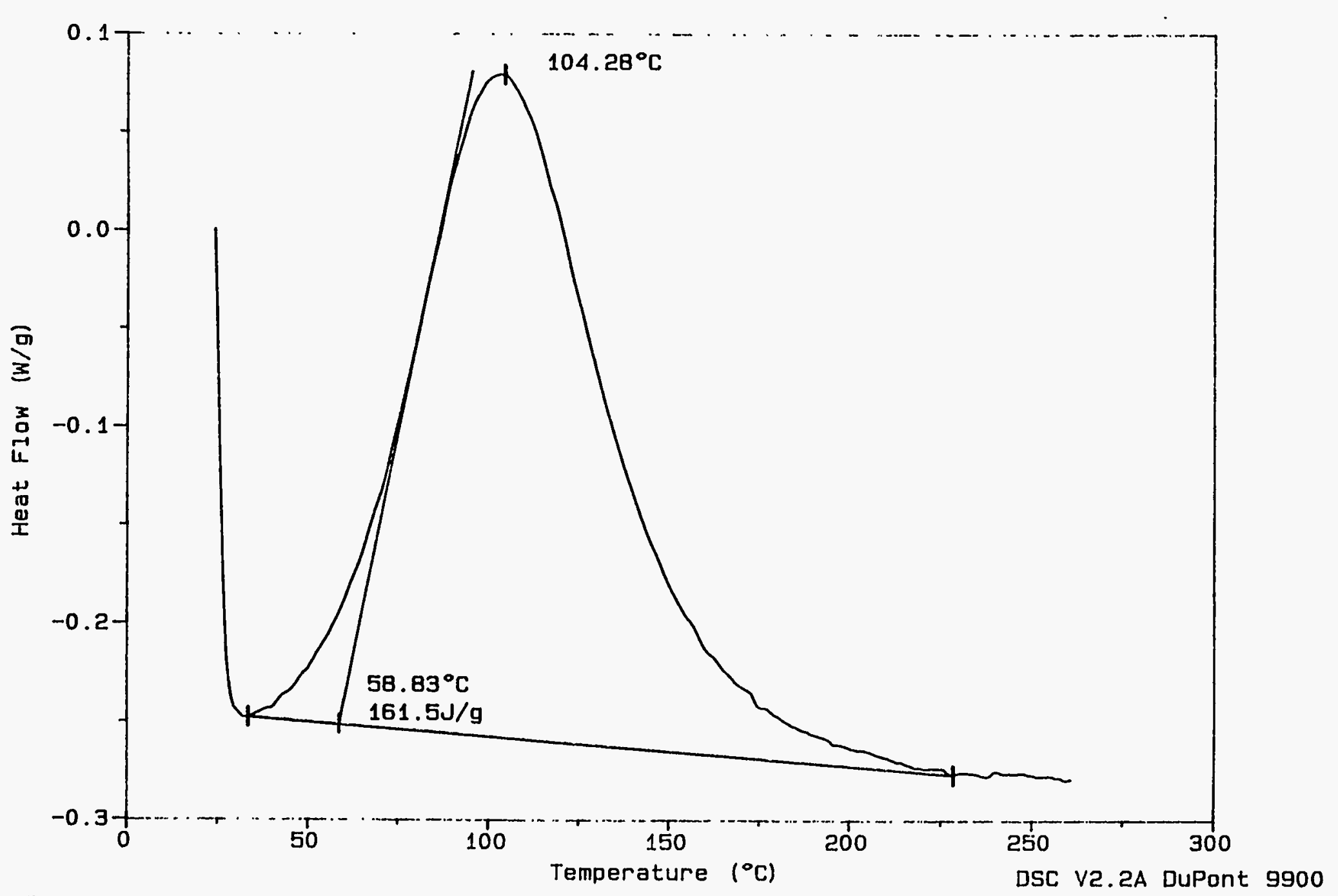

File: DOI1.29

Operator: B. Parker

Run Date: 02/18,'92 06: 13

Figure 1-5. Heat Flow as a Function of Temperature at $8^{\circ} \mathrm{C} / \mathrm{min}$ for EC2216 


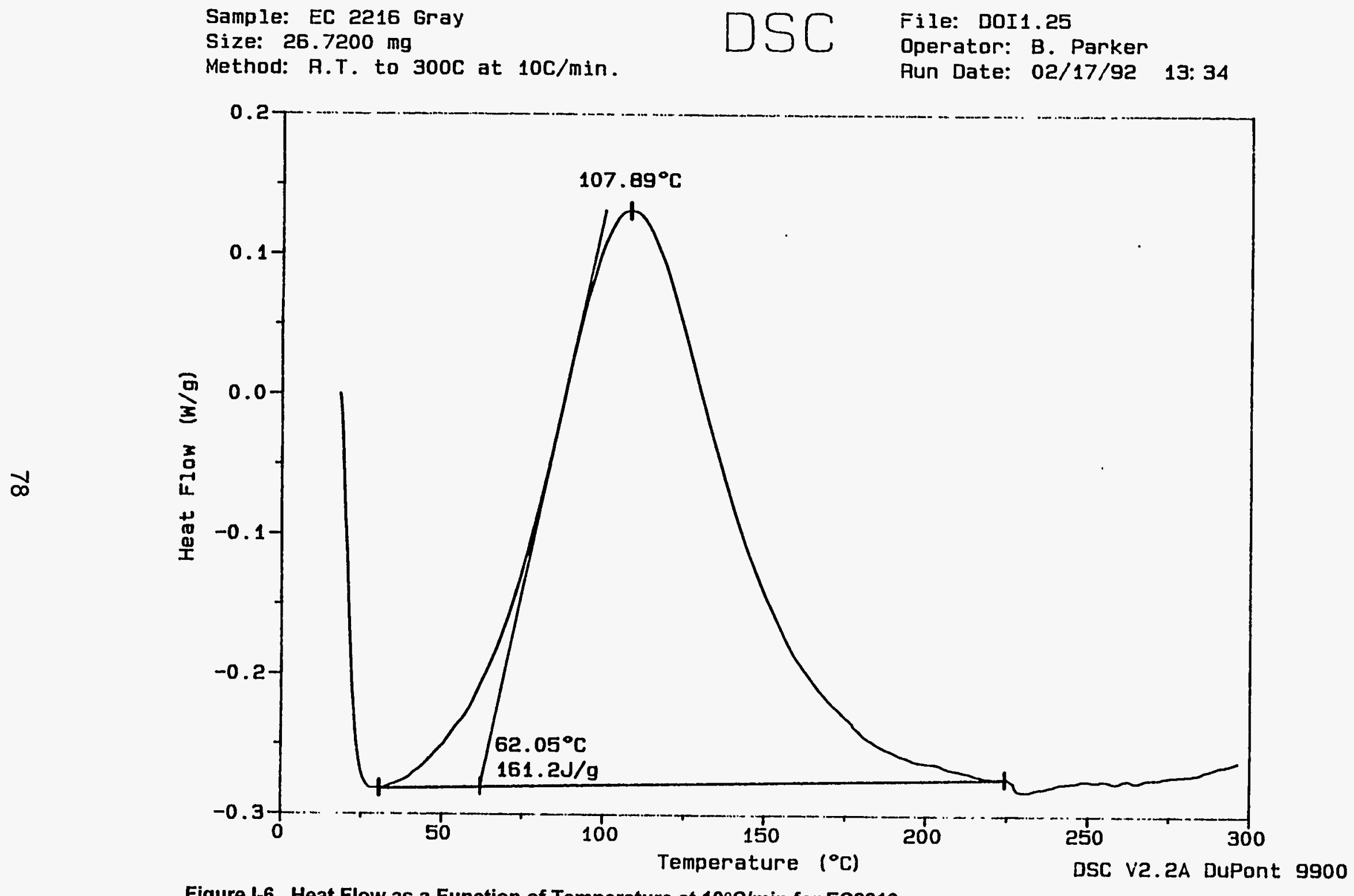

Figure 1-6. Heat Flow as a Function of Temperature at $10^{\circ} \mathrm{C} / \mathrm{min}$ for EC2216 
Sample : EC 2216 Gray

$D S C \quad \begin{aligned} & \text { Operator: B. Parker } \\ & \text { Pun Date: } 02 / 17 / 92\end{aligned}$

\section{Comment:}

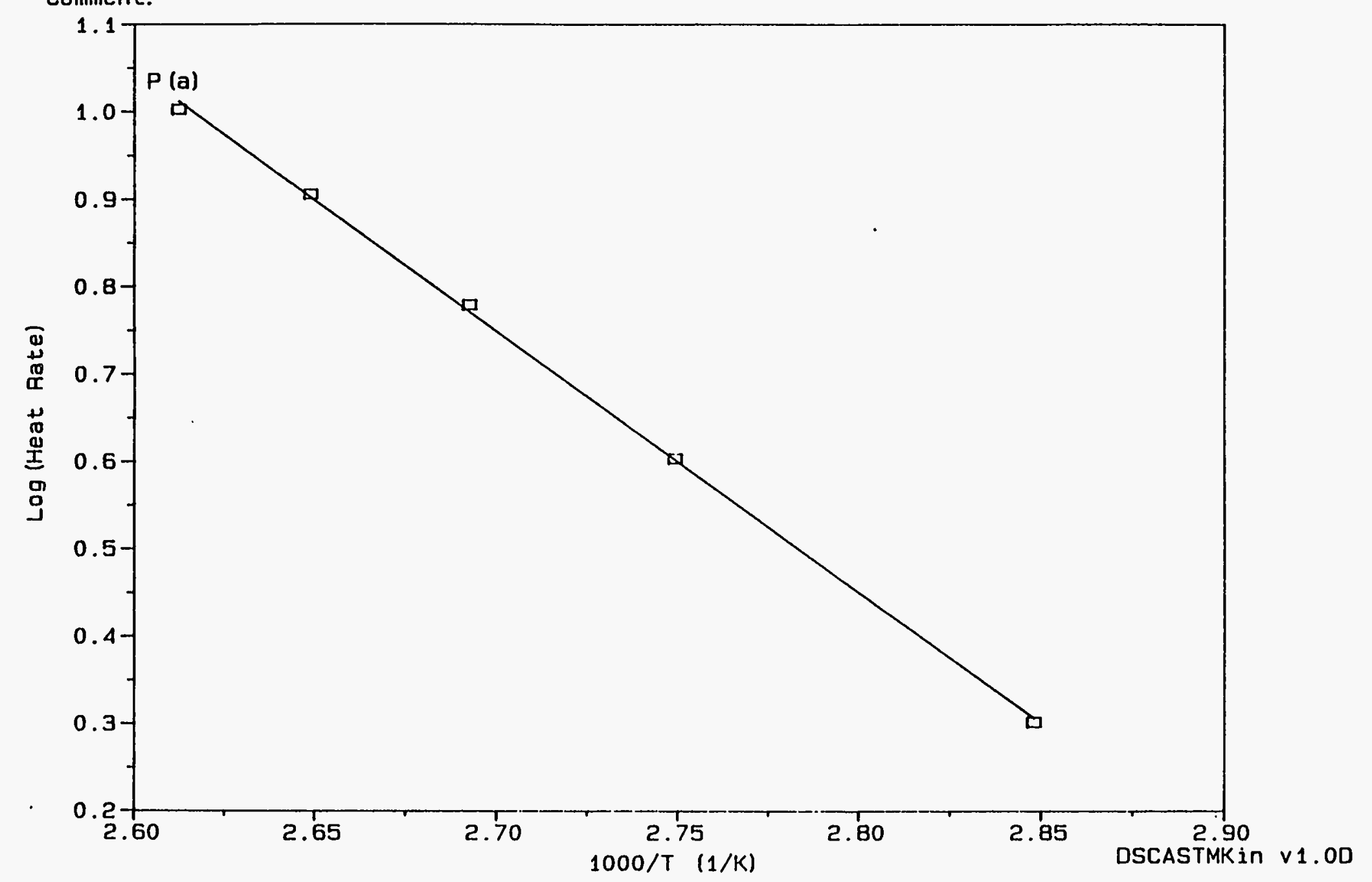

Figure 1-7. Log of the Heat Rate Versus the Reciprocal of the Temperature for EC2216 
Sample : EC 2216 Gray

$\square S C \begin{aligned} & \text { Operator: B. Parker } \\ & \text { Run Date: 02/17/92 }\end{aligned}$

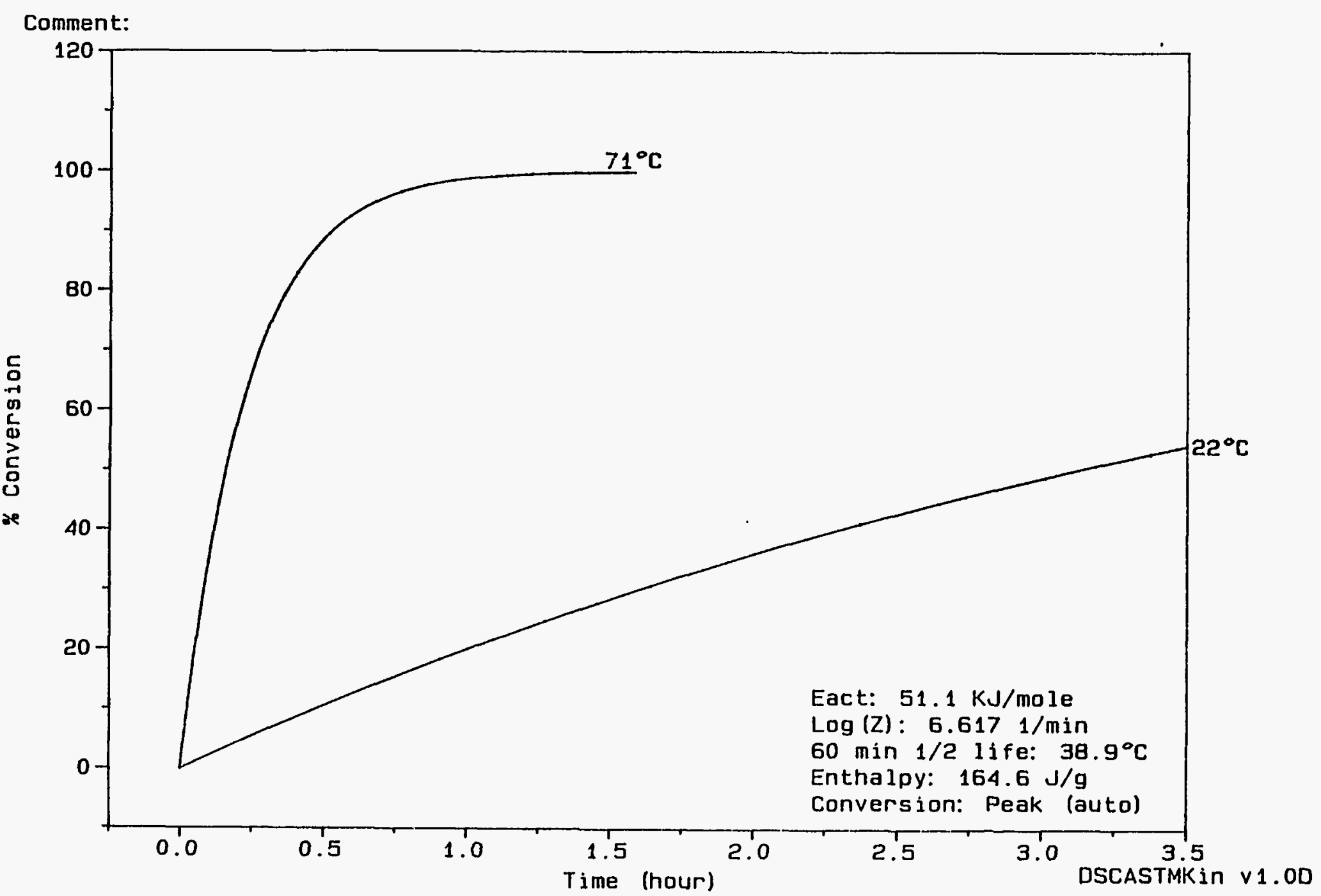

Figure 1-8. Predicted Cure Rate at $71^{\circ} \mathrm{C}$ and $22^{\circ} \mathrm{C}$ for EC2216 
Sample : EC 2216 Gray

$\square \circlearrowleft\left[\begin{array}{l}\text { Operator: B. Parker } \\ \text { Aun Date: } 02 / 17 / 92\end{array}\right.$

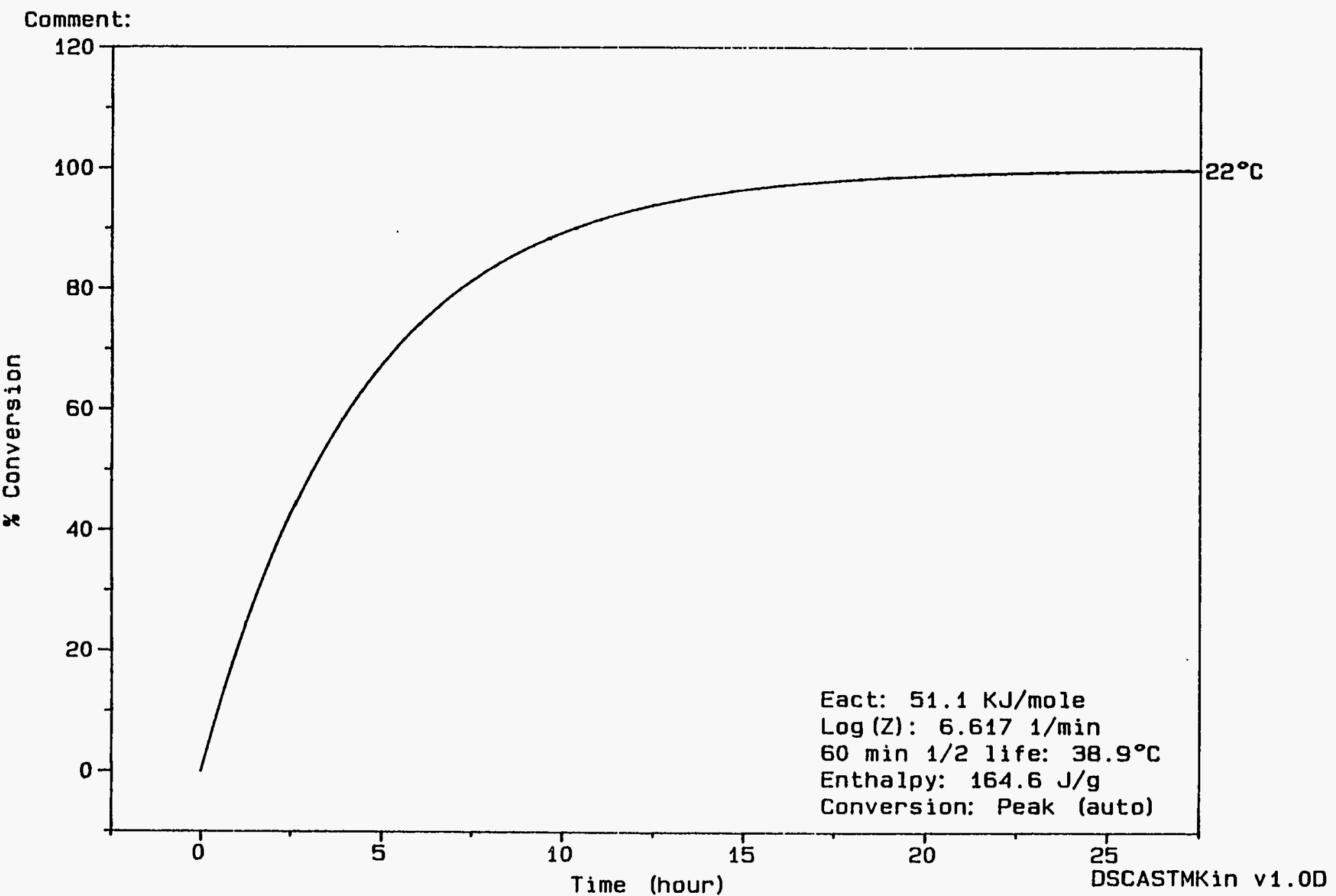

Figure 1-9. Predicted Cure Rate at $22^{\circ} \mathrm{C}$ and Measured Value for EC2216 
Appendix J

Thermal Conductivity 


\section{Thermal Conductivity}

\section{Test Purpose:}

The purpose of this test is to determine the thermal conductivity of a material using the guarded-comparative-longitudinal heat flow technique on the optical adhesives .

\section{Background:}

The testing is per ASTM E 1225 and is included in Appendix L. This test method describes a steady state technique for the determination of the thermal conductivity, $\mathrm{X}$, of homogeneous-opaque solids. This test method is for materials with effective conductivities in the approximate range of $.12<\mathrm{X}<116 \mathrm{BTU} / \mathrm{hr}-\mathrm{ft}-{ }^{\circ} \mathrm{F}$ over the approximate temperature range between -300 and $1550^{\circ} \mathrm{F}$ which is appropriate for the optical adhesives.

\section{Sample Preparation:}

Samples were molded into a two-inch diameter by one-inch tall cylinders in RTV molds and cured per Table A- 1 in Appendix A. After curing, a 0.020 inch by 0.020 inch groove was machined on each flat side parallel to each other. A thermal couple was RTV'ed in place in both grooves.

\section{Test Procedure:}

The samples were prepared and tested in accordance with ASTM E 1225. The thermal conductivity tests were performed using a Dynatech Model TCFCM-N20 comparative thermal conductivity instrument. A test specimen is inserted under load between two similar specimens of a material of known thermal properties. A temperature gradient is established in the test stack and heat losses minimized by use of a longitudinal guard having approximately the same temperature gradient conditions. At equilibrium conditions, the thermal conductivity is derived in terms of the temperature gradients in the respective specimens and the thermal conductivity of the reference materials.

\section{Test Results:}

Table $\mathrm{J}-1$ shows the thermal conductivity at $20^{\circ} \mathrm{C}$. Figures $\mathrm{J}-1$ through $\mathrm{J}-6$ show the thermal conductivity as a function of temperature (divide the values by 12 to get the units in BTU/hr-ft- ${ }^{\circ} \mathrm{F}$ ). Figure J-6 shows the test data for a pyocerm 9606 reference standard. The deviation between actual and measured thermal conductivity ranged from 0.2 to 2.4 percent high for the measured data. 
Table J-1: Thermal Conductivity at $20^{\circ} \mathrm{C}$

\begin{tabular}{|c|c|}
\hline Material & $\begin{array}{l}\text { Thermal Conductivity } \\
\left(\text { at } 20^{\circ} \mathrm{C} \text { ) BTU/hr-ft- }{ }^{\circ} \mathrm{F}\right.\end{array}$ \\
\hline A121:1 & 0.1978 \\
\hline A12 2:3 & 0.2262 \\
\hline A12 1:2 & 0.1918 \\
\hline EC 2216 (gray) & 0.2468 \\
\hline EA 9309.3 NA & 0.1474 \\
\hline
\end{tabular}




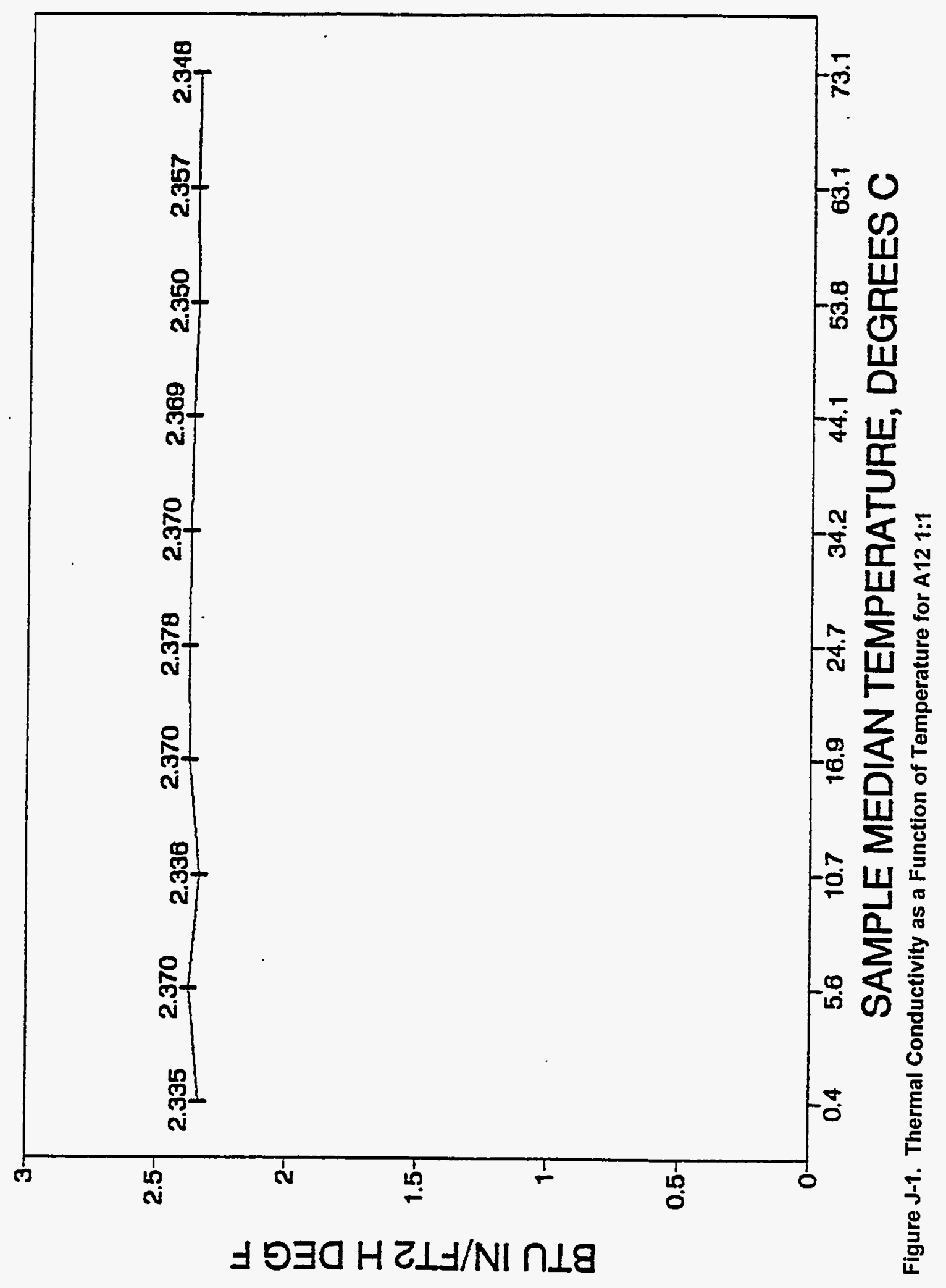




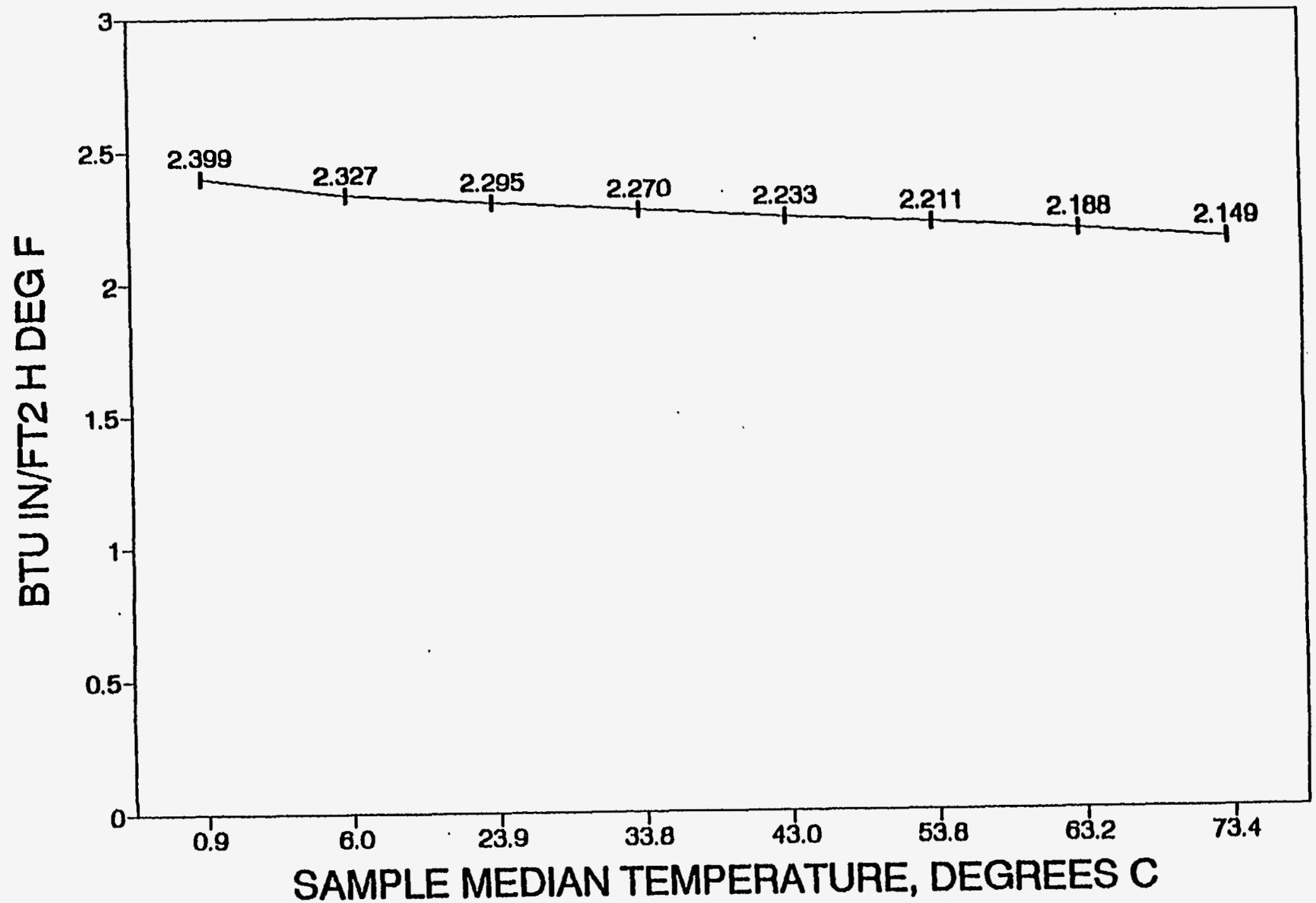

Figure J-2. Thermal Conductivity as a Function of Temperature for A12 1:2 


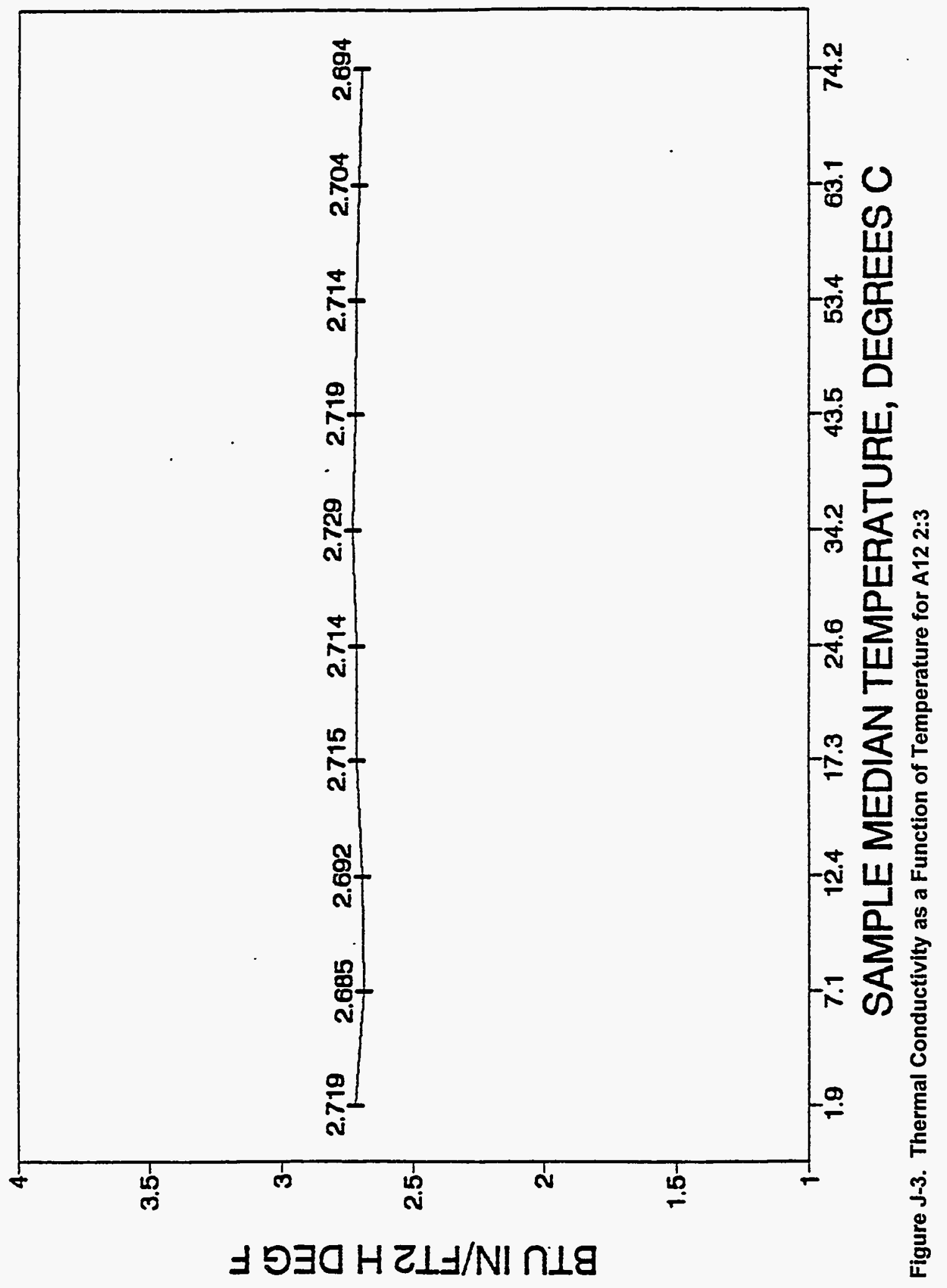




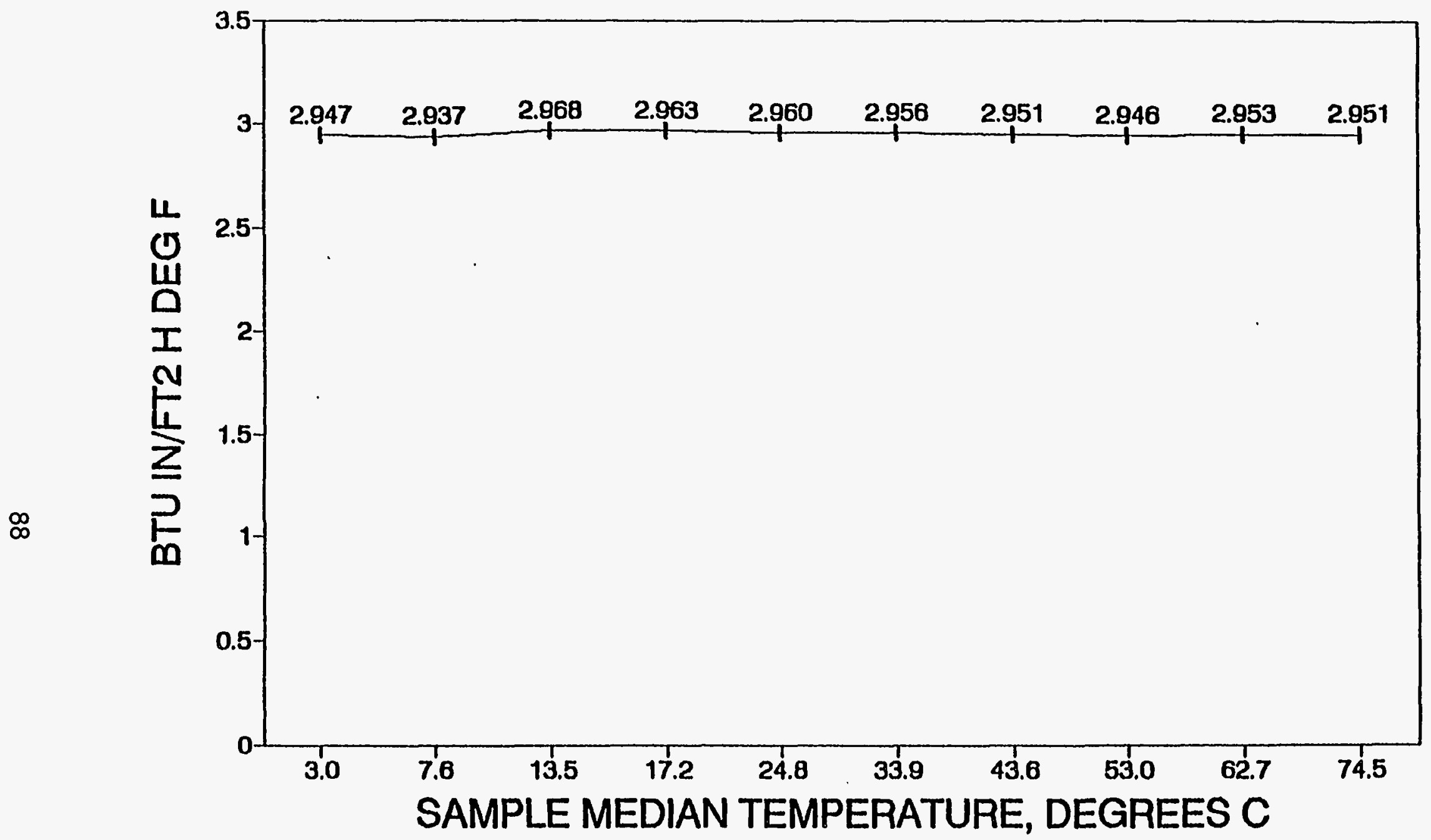

- - ACTUAL DATA

Figure J-4. Thermal Conductivity as a Function of Temperature for EC2216 


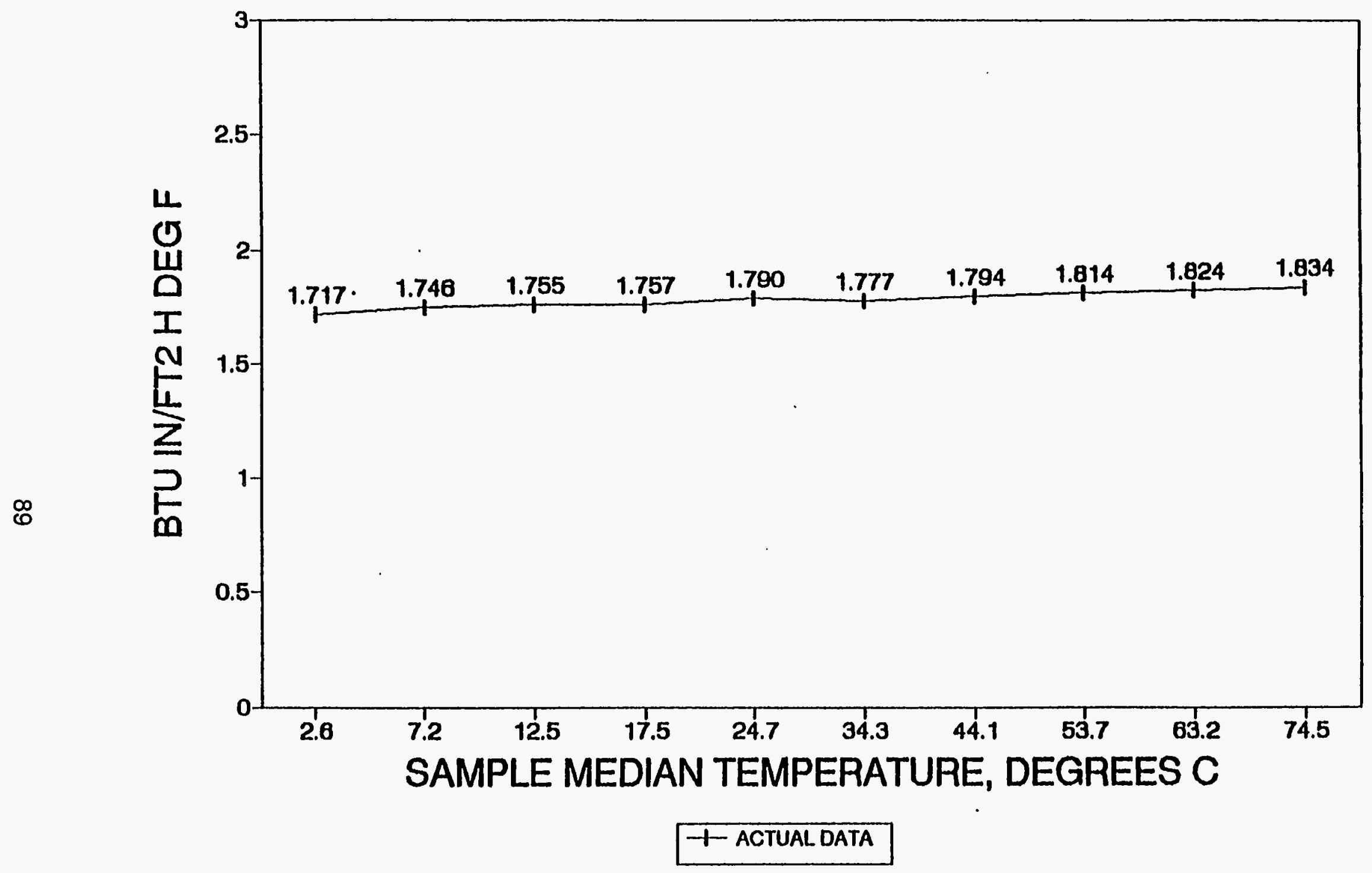

Figure J-5. Thermal Conductivity as a Function of Temperature for EA9309.3 NA 


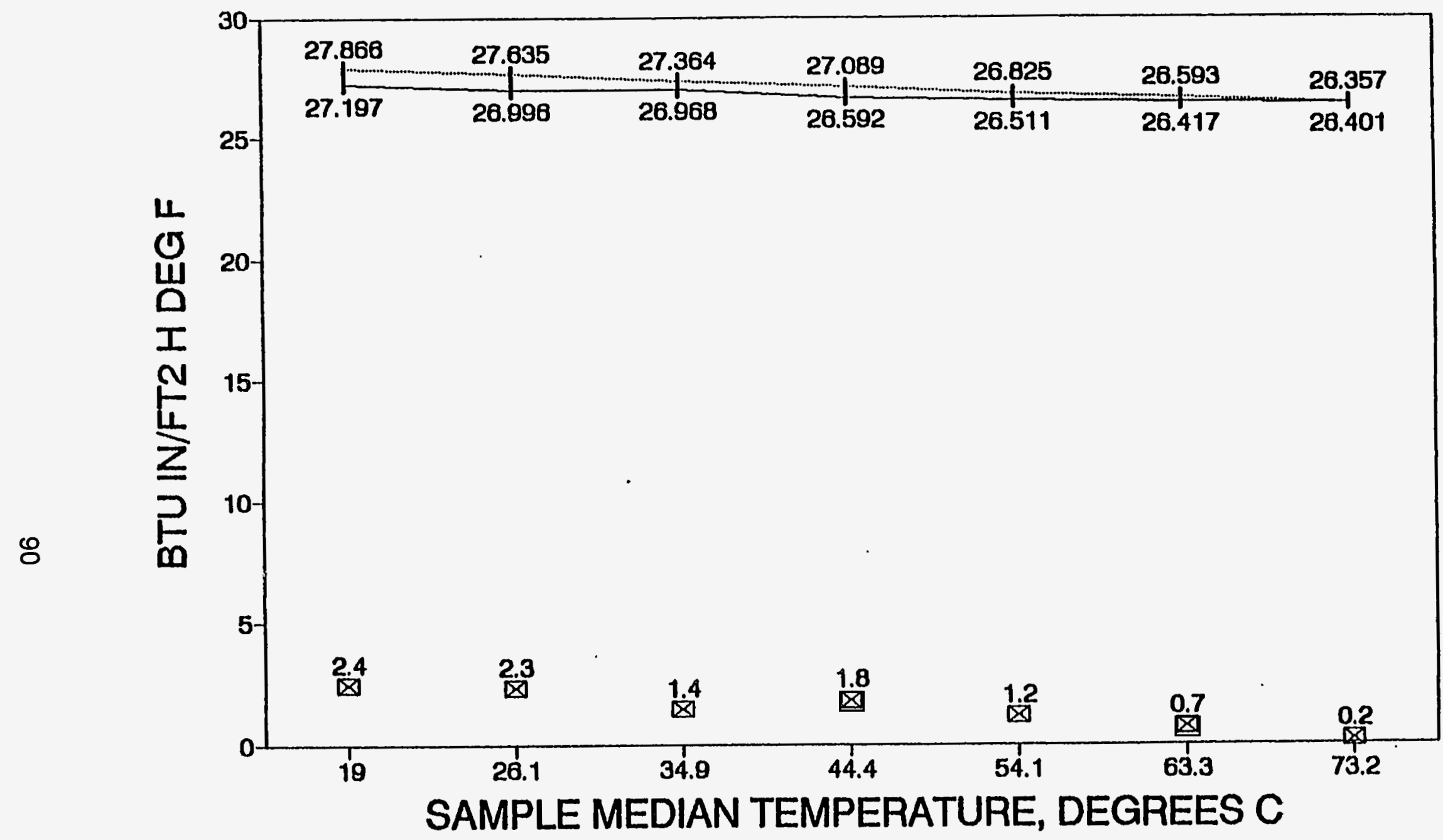

I ACTUAL DATA

-.... PYOCERM REF DATA $\otimes \%$ DIFFERENCE

Figure J-6. Pyrocerm 9606 Reference Standard 
Appendix K

Shrinkage and Density 


\section{Shrinkage and Density}

\section{Test Purpose:}

The purpose of this test is to determine the shrinkage after curing and the density of the optical adhesives.

\section{Background:}

To determine the shrinkage of adhesives after curing one must use caution to get the correct results. Shrinkage determination is made by measuring the mold in which the material will be cured and the dimensions of the final cured specimen. Adhesives, being what they are, have a tendency to bond to the mold, giving erroneous results. Typically, this is corrected by lining the mold with polyethylene film so that the adhesives do not bond to the mold. The film is removed after curing, and the specimens are then measured (the thickness of the film is accounted for in the baseline measurements of the mold). In this determination, an ASTM mold was not available, and RTV silicon tensile molds were used. The RTV silicon mold was flexible and would move with the adhesive while it cured.

The density measurements were performed per ASTM D 792 and is included in Appendix L.

\section{Sample Preparation:}

Samples were mixed and cured per Table A-1 in Appendix A.

\section{Test Procedure:}

Shrinkage data was obtained using 15 molded tensile test specimens for each formulation. The specimens were approximately 7.018 inches long and 0.752 inch wide and were machined to a final thickness of 0.125 inch. Measurements were made using a digital caliper for length and a digital micrometer for width. The linear shrinkage percent is calculated by dividing the length of the cured material by the length of the mold. Since the final thickness of the tensile specimens were machined to 0.125 inch, the volume shrinkage was calculated by dividing the volume of the sample (length $x$ width $^{2}$ ) and dividing by the volume of the mold (length $x$ width ${ }^{2}$ ).

Density (or Specific Gravity) measurements were performed per ASTM D 792 test method $A$. This test method involves weighing a one-piece specimen of one to 50 grams in air and in water (using a sinker with plastics that are lighter than water). 
This test method is suitable for plastics that are wet by, but not affected by water. Two samples of each formulation were measured.

\section{Test Results:}

Table K-1 shows the results of the shrinkage and density for the cured optical adhesives.

Table K-1: Linear Shrinkage, Volume Shrinkage, Density, and Specific Gravity

\begin{tabular}{||l|c|c|c|c||}
\hline \hline Material & $\begin{array}{c}\text { Linear } \\
\text { shrinkage } \\
\text { (percent) }\end{array}$ & $\begin{array}{c}\text { Volume } \\
\text { Shrinkage } \\
\text { (percent) }\end{array}$ & Density & $\begin{array}{c}\text { Specific } \\
\text { (lbs./in3) }\end{array}$ \\
\hline \hline A12 1:1 & 1.32 & 4.20 & 0.0497 & 1.375 \\
\hline A12 2:3 & 1.27 & 4.35 & 0.0491 & 1.360 \\
\hline A12 1:2 & 1.26 & 3.83 & 0.0484 & 1.340 \\
\hline EC 2216 (gray) & 1.40 & 4.06 & 0.0493 & 1.366 \\
\hline EA 9309.3 NA & 1.12 & 4.30 & 0.0423 & 1.170 \\
\hline
\end{tabular}


Appendix L

ASTM Standards 


\title{
Standard Test Method for Tensile Strength of Molded Electrical Insulating Materials ${ }^{1}$
}

\begin{abstract}
This standard is issued under the fixed designation D 651: the number immediately following the designation indicates the year of original adoption or, in the case of revision. the year of last revision. A number in parentheses indicates the year of last reapproval. A superscript epsilon (c) indicates an editorial change since the last revision or reapproval.

This lest method has been approved for use by agencies of the Depanment of Defense and for listing in the DoD index of Specticallons and Standards.

The commilue responsible for this standard has weted its withdrawal. In the ahsence of subsaantial reasons that it should be contunued. the Soctety will approve wuthdrawal from publication in June 1091
\end{abstract}

\section{Scope}

1.1 This test method covers the general procedure for determining the tensile strength of various molded electrical insulating materials. However, modifications in the procedure may be desirable for different types of such materials, and existing ASTM methods covering the requirements for testing a given material should be consulted for special details that may apply.

1.2 The values stated in inch-pound units are to be regarded as the standard.

1.3 This standard may involve hazardous materials, operations, and equipment. This standard does not purport to address all of the safety problems associated with its use. It is the responsibility of whoever uses this standard to consult and establish appropriate safety and health practices and determine the applicability of regulatory limitations prior to use.

\section{Referenced Documents}

\subsection{ASTM Standards:}

D618 Methods of Conditioning Plastics and Electrical Insulating Materials for Testing

D638 Test Method for Tensile Properties of Plastics 2

D 647 Practice for Design of Molds for Test Specimens of Plastic Molding Materials ${ }^{2}$

D 731 Test Method for Molding Index of Thermosetting Molding Powder ${ }^{2}$

D 1896 Practice for Transfer Molding Specimens of Thermosetting Compounds ${ }^{3}$

D 3123 Test Method for Spiral Flow of Low-Pressure Thermosetting Molding Compounds ${ }^{4}$

\section{Apparatus}

3.1 Any universal testing machine may be used, provided it is accurate to $1 \%$ of the lowest load to be applied. Special specimen holders made of steel, as shown in Fig. 1, shall be used for such test specimens as are of a shape to fit them. The specimen holders shall be attached to the heads of the testing machine by links held so that the pull is central, to avoid any

' This test method is under the jurisdiction of ASTM Committee D-9 on Electrical and Electronic Insulating Materiats and is the direct responsibility of Subcommittee D09.07 on Flexible and Rigid Insulating Matenals.

Current edition approved Dec. 28. 1984. Published February 1985. Onginally

published as D 6SI - $41 \mathrm{~T}$. Last previous edition D 651 -80 .

=.tnnutal Bunk or.ASTit Standards. Vol 08.0t

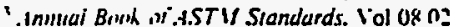

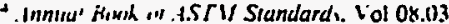

transverse strain. Equally suitable holders. suitably mounted. shall be used for specimens of other shapes.

\section{Test Specimen}

4.1 Compression Molded-Unless otherwise required, the test specimen for hot-molded and plastic cold-molded materials shall be molded to the form and dimensions shown in Fig. 2, the use of either the $1 / 8-i n$. (3.2-mm) or the 1/4-in. $(6.3-\mathrm{mm})$ specimen being permissible. Values for tensile strength obtained with these two specimens may be unequal. and in such cases the specimen which is considered most representative of the material and its intended use shall be tested.

4.2 Transfer or Injection Molded-Soft flow materials (see Test Methods D 731 and $D$ 3123) shall be molded to the dimensions of Fig. 1 in Test Method D 638.

\section{Mold}

5.1 Compression Molded-The specimens shall be molded in molds conforming to Fig. 3 of Practice D 647. The pressure and other molding conditions used in molding test specimens shall be agreed upon by the seller and the purchaser.

5.2 Transfer or Injection Molded-The specimens shall be molded in molds conforming to the tension cavity shown in Fig. 7 of Practice D 647. The pressure and other molding conditions used in molding test specimens shall be agreed upon by the seller and the purchaser.

\section{Conditioning}

6.1 Unless otherwise specified, all specimens prior to testing shall be conditioned in accordance with Methods D 618, the procedure used to be agreed upon between the supplier and purchaser.

\section{Test Conditions}

7.1 Tensile strength tests for materials conditioned (Section 6) by Procedure $A$ shall be performed at $23 \pm 1^{\circ} \mathrm{C}$ $\left(73.4 \pm 1.8^{\circ} \mathrm{F}\right)$ and $50 \pm 2 \%$ relative humidity. For materials conditioned by Procedure B, these tests shall be performed at $23 \pm 1^{\circ} \mathrm{C}\left(73.4 \pm 1.8^{\circ} \mathrm{F}\right)$ and the humidity prevailing in the laboratory. If, because of lack of equipment. matenals conditioned by Procedures A and B cannot be tested undet controlled humidity, the effect of exposure between cond: tioning the testing shall be established and reported. 


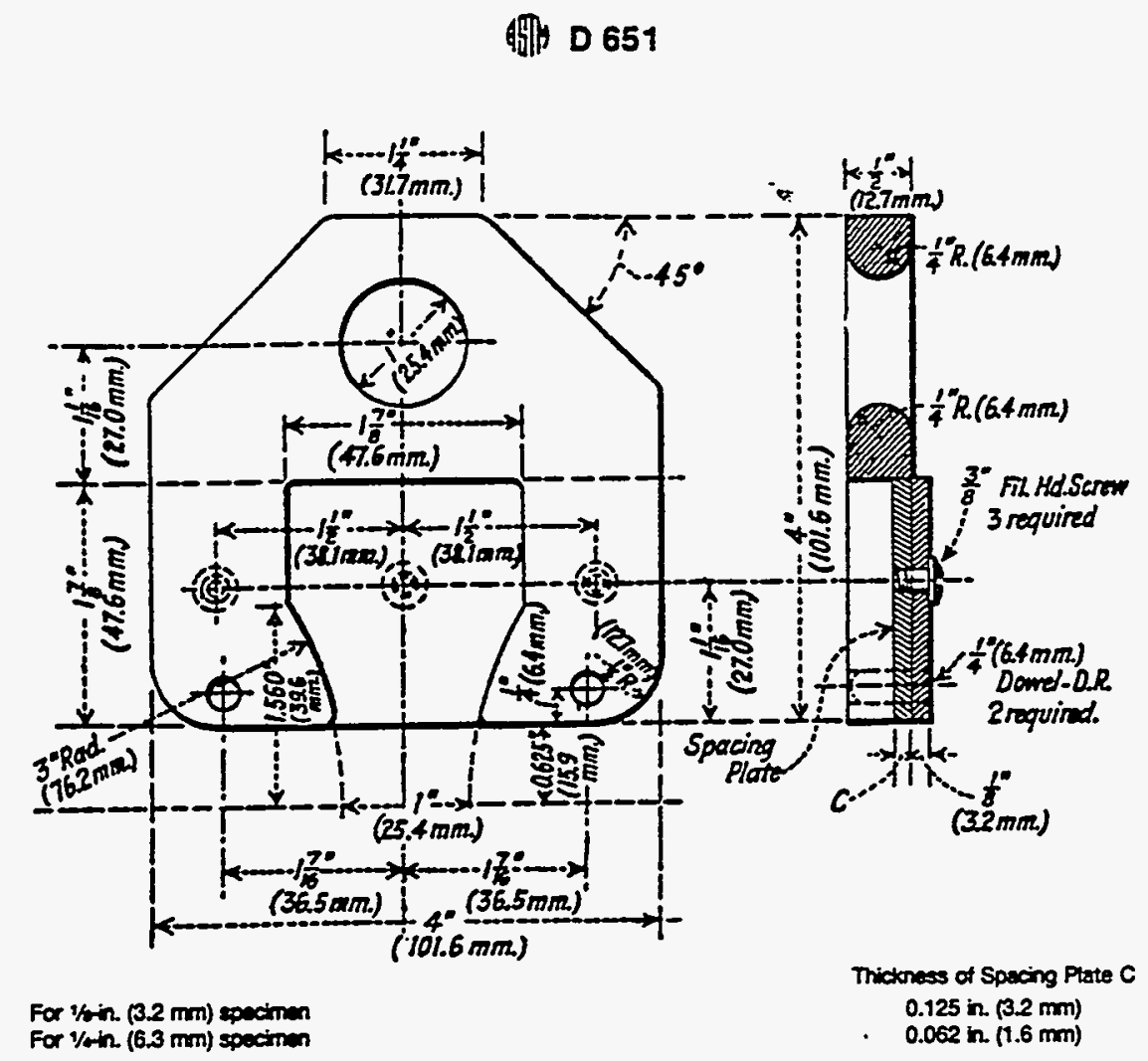

FIG. 1 Spocimen Holder

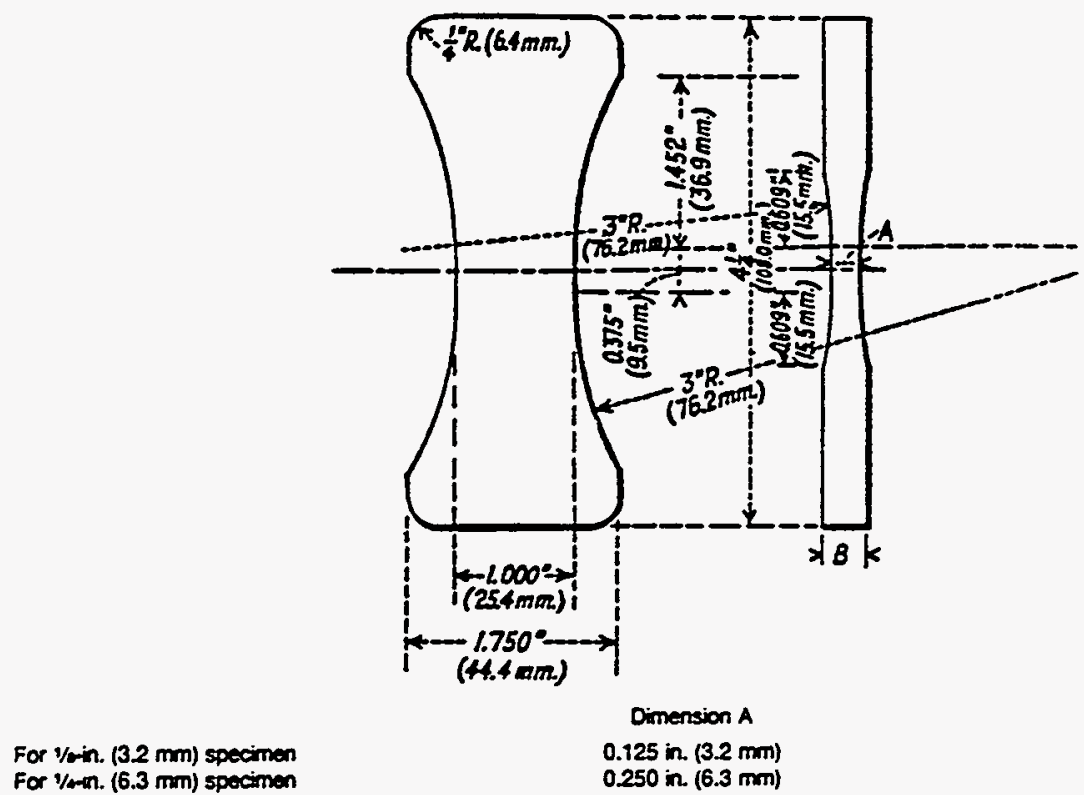

NoTE-To permit the use of one mold tor all molding compounds. the following vanations in dimensions of the test specamen will be permussible:

For hol-molded compounds
For cold-molded compounds

For cold-molded compounds

FG. 2 Tension Test Specimen 


\section{Procedure}

8.1 Five conditioned specimens shall be tested for each type of molded material, each specimen being properiy aligned in the holders and broken. The crosshead speed of the testing machine shall be such that the load can be accurately weighed, but shall not exceed 0.050 in. (1.27 $\mathrm{mm}) / \mathrm{min}$ with the machine running idle.

9. Report

9.1 The report shall include the following:

9.1.1 The breaking load of each specimen in pounds or kilograms.

9.1.2 The thickness and width of each specimen in inches or millimetres, as measured by a micrometer at the center of the specimen. that is, the point of minimum section,

9.1.3 The ultimate tensile strength in pounds-force per square inch or in mega-pascals of each specimen, calculated from the minimum area measured at the center of the test specimen before the load is applied.
9.1.4 The character of the material tested. with a descrip. tion of the fracture and its location with respect to the middle point of the specimen,

9.1.5 The speed in inches or millimetres per minute at which the head of the testing machine traveled during the test,

9.1.6 The elongation in inches or millimetres if determined,

9.1.7 Conditioning procedure prior to testing, and

9.1.8 In the case of tests performed after conditioning but not in a controlled atmosphere, reference shall be made to the availability of data showing the effects of exposure between conditioning and testing, and the test conditions observed.

\section{Precision and Bias}

10.1 The precision of this method has not been determined. A statement of bias is unavailable in view of the lack of a standard reference material for this property.

The American Sociery for Testing and Materiats takes no position respecting the velidity of any perent rignts asserted in connection with any ttem mentionod in thls standard. Users of this standard ere expresely actised the determination of the validity of any such patem nghts, and the riak of infringement of such rights, are entirely their own responsibility.

This standerd is subject to revision at any time by the responsiblo fochnical commitios and mest bo reviewed every five yaers and

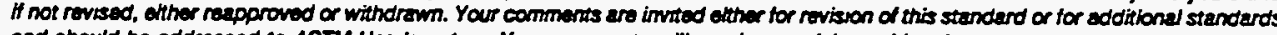
and should be addressed to ASTM Headquarters. Your comments will recaive careful consideretion at a meating of the responsible tochical committee, which you may attend. If you feel that your comments heve not recoived a feir hoering you should make your views known to the ASTM Commites on Stendends, 1916 Race St, Philedaphian, PA 19103. 


\title{
Standard Test Method for Compressive Properties of Rigid Plastics ${ }^{1}$
}

\begin{abstract}
This seandard is issued under the fixed designation D 695: the aumber immediately following the decipartion indicates the year of original adoption or, in the exse of revision, the year of last revision. A number in parentbeses iodicales the year of lass reapproval. A superseript epsilon (t) isdiertes an editorial chanes sioce the les revision or reappovel.

This test method has been approved for use by agencies of the Deparment of Defense to replace Mectod 1021. Consuls the DaD Index of Sperifications and Standards for the specific year of issue which has been adoped by the Department of Defense.
\end{abstract}

\section{Scope}

1.1 This test method covers the determination of the mechanical properties of unreinforced and reinforced rigid plastics, including high-modulus composites, when loaded in compression at relatively low uniform rates of straining or loading. Test specimens of standard shape are employed.

1.2 The values stated in SI units are to be regarded as the standard. The values in parentheses are for information only.

NoTE 1-A complete metric companion to Tex Method D 695 hro been developed-D 695M.

Note 2-For compressive properties of resin-matrix composites reinforced with oriented continuous, discontinuous, or cross-ply reinforcements, tests may be made in accordance with Tes Method D 3410 .

1.3 This standard does not purport to address all of the safety problems associated with its use. It is the responsibility of the user of this standard to establish appropriate safety and health practices and determine the applicability of regulatory limitations prior to use.

\section{Referenced Documents}

\subsection{ASTM Standards:}

D618 Methods of Conditioning Plastics and Electrical Insulating Materials for Testing

D638 Test Method for Tensile Properties of Plastics ${ }^{2}$

D 695M Test Method for Compressive Properties of Rigid Plastics [Metric] ${ }^{2}$

D 3410 Test Method for Compressive Properties of Unidirectional or Crossply Fiber-Resin Composites ${ }^{3}$

D 4066 Specification for Nylon Injection and Extrusion Materials

E 4 Practices for Load Verification of Testing Machiness

E 83 Practice for Verification and Classification of Extensometers's

E 691 Practice for Conducting an Interlaboratory Study to Determine the Precision of Test Method ${ }^{6}$

\section{Terminology}

3.1 Definitions.

3.1.1 compressive deformation-the decrease in length

'This tex method is under the juristiction of ASTM Commitree D-20 on Pharies and is the direct responsibility of Subcomarize D20.10 on Mectanical Properties.

Curremt edition approved October 26, 1990. Published December 1990.

Originally published as D 695 - 42 T. Las previous edition D $695-89^{* 1}$.

I Annual Boot of ASTM Slandards. Vol 08.01.

3 Annual Boak of ASTM Siandards Val 15.03 .

Annual Book of ASTM Standards. Vol 08.03.

S Annual Book of ASTM Standards, Vol 03.01.

- Annual Book of ASTM Slandards, Vol 14.02 produced in the gage length of the test specimen by a compressive load. It is expressed in units of length.

3.1.2 compressive strain-the ratio of compressive defor mation to the gage length of the test specimen, that is, the change in length per unit of original length along the longitudinal axis. It is expressed as a dimensionless ratio.

3.1.3 compressive strengh $h$-the maximum compressive stress (nominal) carried by a test specimen during a compres. sion test. It may or may not be the compressive stress (nominal) carried by the specimen at the moment of rupture.

3.1.4 compressive strength at failure (nominal) - the com. pressive stress (nominal) sustained at the moment of failure of the test specimen if shattering occurs.

3.1.5 compressive stress (nominal) - the compressive load per unit area of minimum original cross section within the gage boundaries, carried by the test specimen at any given moment. It is expressed in force per unit area.

NOTE 3-The expression of comprestive properties in terms of the minimum original cross section is almost universelly used. Under some circumstances the compressive propertie have bees expressed per unit of preveiling cross section. These properies are called "true" compres. sive properties

3.1.6 compressive stress-strain diagram-a diagram in which values of compressive stress are plotted as ordinates against corresponding values of compressive strain as abscissas.

3.1.7 compressive yield point-the first point on the stress-strain diagram at which an increase in strain occurs without an increase in stress.

3.1.8 compressive yield strength-normally the stress at the yield point (see also 3.11).

3.1.9 crushing load-the maximum compressive force applied to the specimen, under the conditions of test, that produces a designated degree of failure.

3.1.10 modulus of elasticity - the ratio of stress (nominal) to corresponding strain below the proportional limit of a material. It is expressed in force per unit area based on the average initial cross-sectional area

3.1.11 offset compressive yield strength-the stress at which the stress-strain curve departs from linearity by $a$ specified percentage of deformation (offser).

3.1.12 percentage compressive strain-the compressive deformation of a test specimen expressed as a percentage of the original gage length.

3.1.13 proportional limit-the greatest stress that a material is capable of sustaining without any deviation from proportionality of stress to strain (Hooke's law). It is expressed in force per unit area.

3.1.14 slenderness ratio-the ratio of the length of $a$ 
column of uniform cross section to its least radius of gration. For specimens of uniform rectangular cross section, the radius of gyration is 0.289 times the smaller crossectional dimension. For specimens of uniform circular cross section, the radius of gyration is 0.250 times the diameter.

\section{Significance and Use}

4.1 Compression tests provide information about the compressive properties of plartics when employed under conditions approximating those under which the tests are made.

42 Compressive properties include modulus of elasticity, ield stress, deformation beyond yield point, and compressir strength (unless the material merely flattens but does not frcture). Materials possessing a low order of ductility may oot exhibit a yield point. In the case of a material that fails in compression by a shattering fracture, the compressive grength has a very definite value. In the case of a material tor does not fail in compression by a shattering fracture, the compressive strength is an arbitrary one depending upon the begree of distortion that is regarded as indicating complete Gilure of the material. Many plastic materials will continue w deform in compression until a flat disk is produced, the rompressive stress (nominal) rising steadily in the process, rithout any well-defined fracture occurring Compressive gength can have no real meaning in such cases.

4.3 Compression tests provide a standard method of thaining data for research and development, quality consol, acceptance or rejection under specifications, and special pupposes. The tests cannot be considered significant for mgineering design in applications differing widely from the load-time scale of the standard tert. Such applications raquire additional tests such as impact, creep, and fatigue.

\section{Apparatus}

5.1 Testing Machine-Any suitable testing machine capeble of control of constant-rate-of-crosshead movement and omprising essentially the following:

5.1.1 Drive Mechanism-A drive mechanism for imarting to the cross-head movable member, a uniform, inotrolled velocity with respect to the base (fixed member), this velocity to be regulated as specified in Section 9.

5.1.2 Load Indicalor-A load-indicating mechanism capable of showing the total compressive load carried by the specimen. The mechanism shall be essentially free from wertia-lag at the specified rate of testing and shall indicate te load with an accuracy of $\pm 1 \%$ of the maximum indicated value of the test (load). The accuracy of the testing jachine shall be verified at least once a year in accordance xith Practices E 4.

5.2 Compressometer-A suitable instrument for deteraining the distance between two fixed points on the test pecimen at any time during the test. It is desirable that this astrument automatically record this distance (or any change $n$ it) as a function of the load on the test specimen. The asrument shall be essentially free of inertia-lag at the pecified rate of loading and shall conform to the requireIents for a Class B-2 extensometer as defined in Practice 583.

Vote 4-The requirements for extensometers cited herein apply to ampressometers as well.

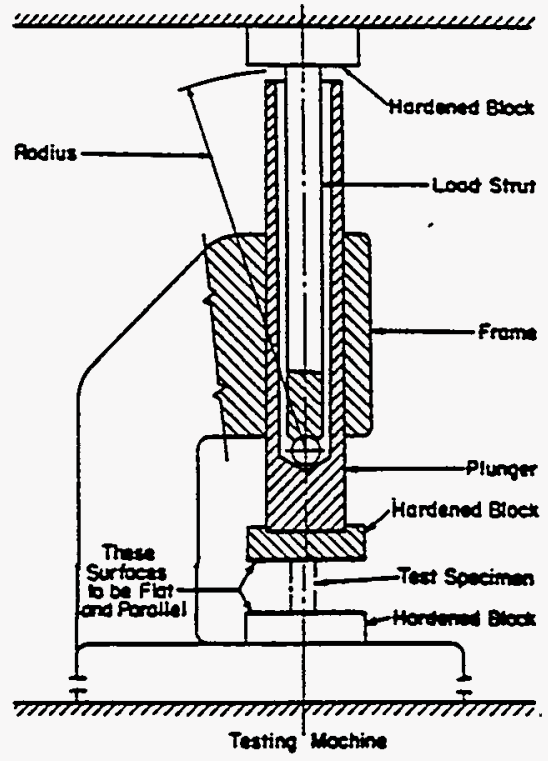

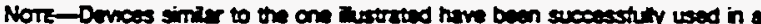
number of differem inboratoms. Detais of the dence develooed at the Nirocn: Burees of Stunderds ere given in the peper by Aitetinsen, C. S. and Milier. J. A. "A Suboress tor Compreserve Tests," Natl. Aovisory Commrties for Aeronautics. Tectricel Noto No. 912. 1943.

FG. 1 Subprese for Comprestion Test

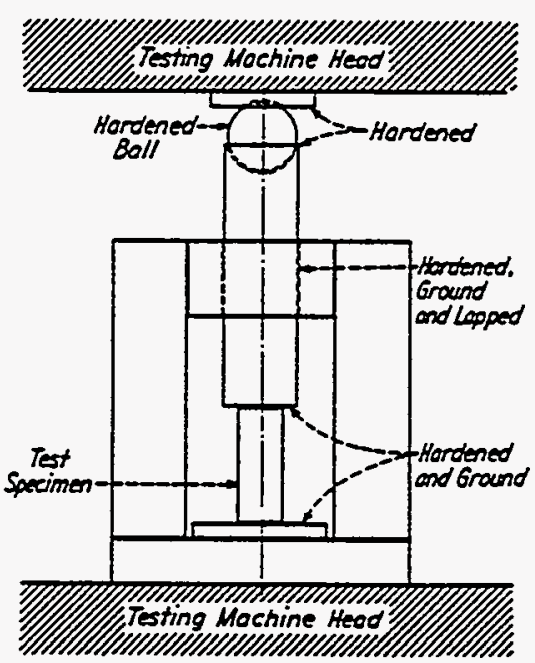

FI. 2 Comprasion Tool

5.3 Compression Tool-A compression tool for applying the load to the test specimen. This tool shall be so constructed that loading is axial within 1:1000 and applied through surfaces that are flat within $0.025 \mathrm{~mm}(0.001 \mathrm{in}$.) and parallel to each other in a plane normal to the vertical loading axis. Examples of suitable compression tools are shown in Figs, 1 and 2.

5.4 Supporing Jig-A supporting jig for thin specimens is shown in Figs. 3 and 4.

5.5 Micrometers-Suitable micrometers, reading to 0.01 


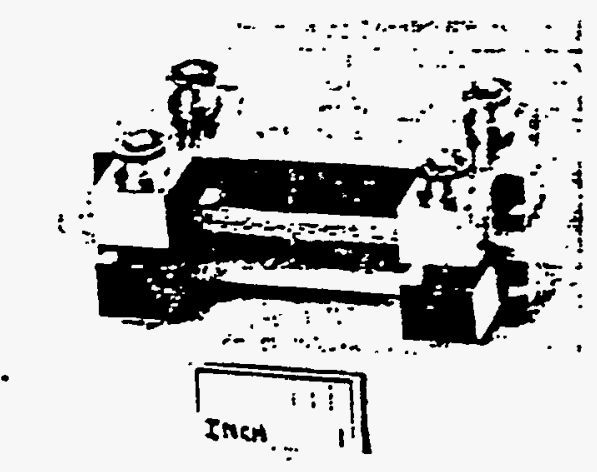

Fic. 3 Support Jig for This Spectimen

$\mathrm{mm}$ or $0.001 \mathrm{in}$. for measuring the width, thickness, and length of the specimens.

\section{Test Specimens}

6.1 Unless otherwise specified in the materials specifications, the specimens described in 6.2 and 6.7 shall be used. These specimens may be prepared by machining operations from materials in sheet, plate, rod, tube, or similar form, or they may be prepared by compression or injection molding of the material to be tested. All machining operations shall be done carefully so that smooth surfaces result. Great care shall be taken in machining the ends so that smooth, llat parallel surfaces and sharp, clean edges to within $0.025 \mathrm{~mm}$ (0.001 in.) perpendicular to the long axis of the specimen, result.

6.2 The standard test specimen, except as indicated in 6.3 to 6.7 , shall be in the form of a right cylinder or prism whose length is twice its principal width or diameter. Preferred specimen sizes are 12.7 by 12.7 by $25.4 \mathrm{~mm}(0.50$ by 0.50 by 1 in.) (prism), or $12.7 \mathrm{~mm}(0.50 \mathrm{in}$.) in diameter by $25.4 \mathrm{~mm}$ (1 in.) (cylinder). Where elastic modulus and offset yield- stress data are desired, the test specimen shall be of such dimensions that the slenderness ratio is in the range from II to 16:1. In this case, preferred specimen sizes are 12.7 b 12.7 by $50.8 \mathrm{~mm}$ ( 0.50 by 0.50 by 2 in.) (prism), or $12.7 \mathrm{~mm}$ $(0.50 \mathrm{in}$.) in diameter by $50.8 \mathrm{~mm}$ ( 2 in.) (cylinder).

6.3 For rod material, the test specimen shall have 3 diameter equal to the diameter of the rod and a sufficient length to allow a specimen slenderness ratio in the range from 11 to 16:1.

6.4 When testing tubes, the test specimen shall have a diameter equal to the diameter of the tube and a length of $25.4 \mathrm{~mm}$ (1 in.) (Note 5). For crushing-load determinations (at right angles to the longitudinal axis), the specimen size shall be the same, with the diameter becoming the height.

NOTE 5-This specimen can be used for tubes with a wall thicknes of $1 \mathrm{~mm}(0.039 \mathrm{in}$.) or over, to inside diameters of $6.4 \mathrm{~mm}(0.25 \mathrm{in}$.) or over, and to outside diameters of $50.8 \mathrm{~mm}$ (2.0 in.) or less.

6.5 Where it is desired to test conventional high-pressure laminates in the form of sheets, the thickness of which is less than $25.4 \mathrm{~mm}$ ( $1 \mathrm{in}$.$) , a pile-up of sheets 25.4 \mathrm{~mm}$ ( 1 in.) square, with a sufficient number of layers to produce a height of at least $25.4 \mathrm{~mm}(1 \mathrm{in}$.), may be used.

6.6 When resting material that may be suspected of anisotropy, duplicate sets of test specimens shall be prepared having their long axis respectively parallel with and normal to the suspected direction of anisotropy.

6.7 Reinforced Plastics. Including High-Strength Compos. ites and High-Strengih Composites and Highly Orthotropic Laminates-The following specimens shall be used for reinforced materials, or for other materials when necessary to comply with the slenderness ratio requirements or to permit attachment of a deformation-measuring device.

6.7.1 For materials $3.2 \mathrm{~mm}(1 / \mathrm{sin}$ ) and over in thickness a specimen shall consist of a prism having a cross section of $12.7 \mathrm{~mm}(1 / 2 \mathrm{in}$.) by the thickness of the material and a length such that the sienderness ratio is in the range from 11 to $16: 1$ (Note 6).
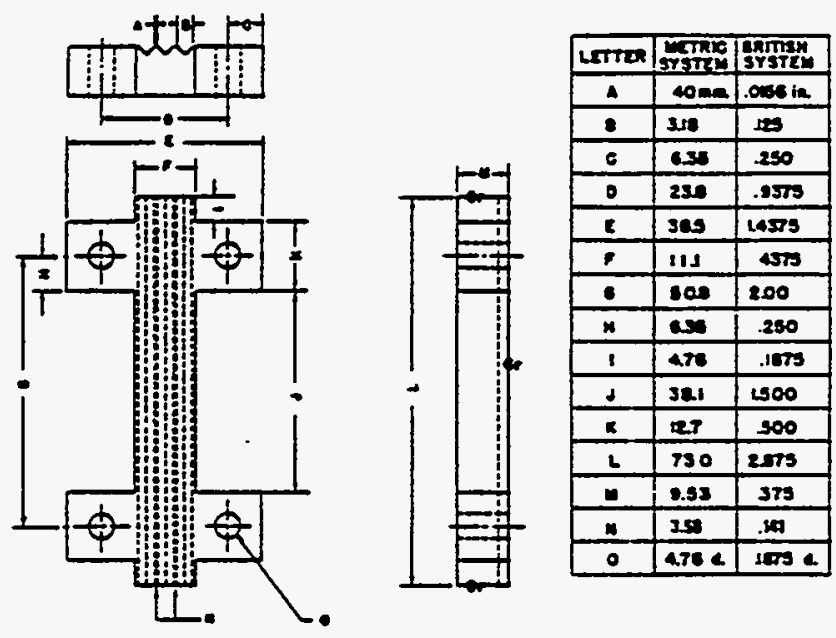

Nor: 1- Cold roled stew

Nore 2-Fumshed four sted mactime screws and nuth, round had, slotted, kngh $31.75 \mathrm{~mm}$ (11/4 in.f Note 3-Gind surtaces denoted "Gr."

FG. 4 Support Jig, Dotails 


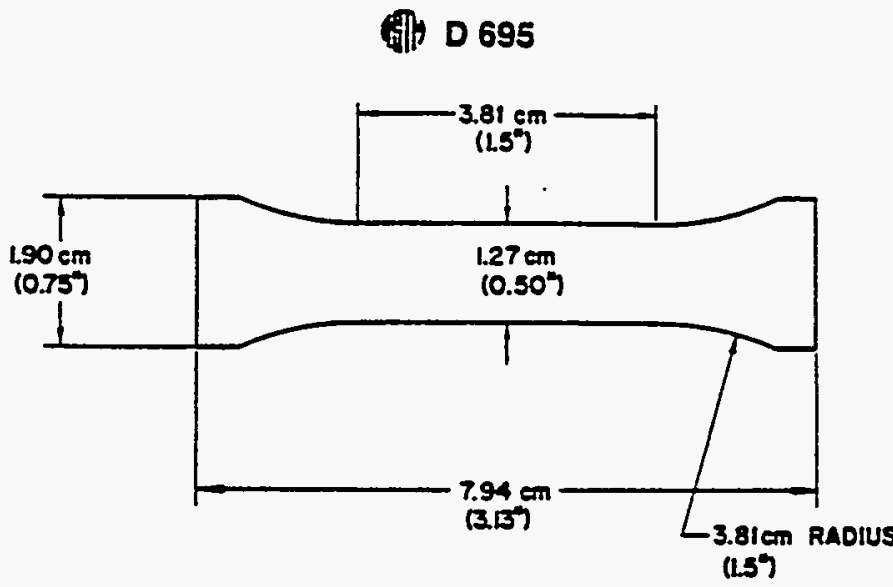

FiC. 5 Comprestion Tost Specimen for Materials Less than $322 \mathrm{~mm}$ Thick

6.7.2 For materials under $3.2 \mathrm{~mm}$ ( $1 / \mathrm{s}$ in.) thick, or where elastic modulus testing is required and the slenderness ratio does not provide for enough length for attachment of a compressometer or similar device, a specimen conforming to that shown in Fig. 6 shall be used. The supporting jig shown in Figs. 3 and 4 shall be used to support the specimen during tert (Note 7).

NOTE 6-If failure for materials in the thickness range of $3.2 \mathrm{~mm}(1 / \mathrm{s}$ in.) is by delamination rather than by the desirable shear piane fracture, the material may be terted in accordance with 6.7.2.

NOTE 7-Round-robin tests have established thas relatively atisfactory measurements of modulus of elasticity may be obrained by applying 2 compressometer to the edges of the jig-supported specimen.

6.8 When testing syntactic foam, the standard test specimen shall be in the form of a right cylinder $25.4 \mathrm{~mm}$ ( 1 in.) in diameter by $50.8 \mathrm{~mm}$ ( 2 in.) in length.

\section{Conditioning}

7.1 Conditioning-Condition the test specimens at $23 \pm$ $2^{\circ} \mathrm{C}\left(73.4 \pm 3.6^{\circ} \mathrm{F}\right)$ and $50 \pm 5 \%$ relative humidity for not less than $40 \mathrm{~h}$ prior to test in accordance with Procedure $A$ of Methods D 618, for those tests where conditioning is required. In cases of disagreement, the tolerances shall be $1^{\circ} \mathrm{C}$ $\left(1.8^{\circ} \mathrm{F}\right)$ and $\pm 2 \%$ relative bumidity.

7.1.1 Note that for some hygroscopic materials, such as nylons, the material specifications (for example, Specification D 4066) call for testing "dry as-molded specimens." Such requirements take precedence over the above routine preconditioning to $50 \% \mathrm{RH}$ and require sealing the specimens in water vapor-impermeable containers as soon as molded and not removing them until ready for testing.

7.2 Test Conditions-Conduct tests in the Standard Laboratory Atmosphere of $23 \pm 2^{\circ} \mathrm{C}\left(73.4 \pm 3.6^{\circ} \mathrm{F}\right)$ and $50 \pm 5 \%$ relative humidity, unless otherwise specified in the test methods. In cases of disagreement, the tolerances shall be $1^{\circ} \mathrm{C}\left(1.8^{\circ} \mathrm{F}\right)$ and $\pm 2 \%$ relative humidity.

\section{Number of Test Specimens}

8.1 At least five specimens shall be tested for each sample in the case of isotropic materials.

8.2 Ten specimens, five normal to and five parallel with the principal axis of anisotropy, shall be tested for each sample in the case of anisotropic materials.

8.3 Specimens that break at some obvious fortuitous flow shall be discarded and retests made, unless such flaws constitute a variable, the effect of which it is desired to study.

\section{Speed of Testing}

9.1 Speed of testing shall be the relative rate of motion of the grips or test fixtures during the test. Rate of motion of the driven grip or fixture when the machine is running idle may be used if it can be shown that the resulting speed of testing is within the limits of variation allowed.

9.2 The standard speed of testing shall be $1.3 \pm 0.3 \mathrm{~mm}$ $(0.050 \pm 0.010 \mathrm{in}$.) $/ \mathrm{min}$, except as noted in 10.5.4.

\section{Procedure}

10.1 Measure the width and thickness of the specimen to the nearest $0.01 \mathrm{~mm}(0.001 \mathrm{in}$.) at several points along its length. Calculate and record the minimum value of the cross-sectional area. Measure the length of the specimen and record the value.

10.2 Place the test specimen between the surfaces of the compression tool, taking care to align the center line of its long axis with the center line of the plunger and to ensure that the ends of the specimen are parallel with the surface of the compression tool. Adjust the crosshead of the testing machine until it just contacts the top of the compression tool plunger.

NOTE 8- The compresion tool may not be necessery for testing of lower modulus (for example, 100000 to $500000 \mathrm{psi}$ ) material if the loading surfaces are maintained smooth. flat, and paralled to the extent that buckling is not incurred.

10.3 Place thin specimens in the jig (Figs. 3 and 4) so that they are flush with the base and centered (Note 9). The nuts or screws on the jig shall be finger tight (Note 10). Place the assembly in the compression tool as described in 5.3.

NoTE 9-A round-robin test, designed to aseess the influence of specimen positioning in the supporting ijg (that is, flush versus centered mounting), showed no signifient effect on compressive scrength due to this variable. However, flush mounting of the specimen with the base of the jis is specified for convenience and ease of mounting Subsrantiating data are filed at ASTM Headquarers (RR:D20-1061).

NoTE 10-A round-robin ters on the effect of laterl pressure at the supporting jig has established that reproducible data can be obtained with the tightness of the jis controlled as indieated.

10.4 If only compressive strength or compressive yield strength, or both, are desired, proceed as follows: 
10.4.1 Set the speed control at $1.3 \mathrm{~mm} / \mathrm{min}(0.050$ in./min) and start the machine.

10.4.2 Record the maximum load arried by the specimen during the test (usually this will be the load at the moment of rupture).

10.5 If stress-strain data are desired, proceed as follows:

10.5.1 Atrach compressometer.

10.5.2 Set the speed control at $1.3 \mathrm{~mm} / \mathrm{min}(0.050$ in./min) and start the machine.

10.5.3 Record loads and corresponding compressive strain at appropriate intervals of strain or, if the test machine is equipped with an automatic recording device, record the complete load-deformation curve.

10.5.4 After the yield point bas been reached, it may be desirable to increase the speed from 5 to $6 \mathrm{~mm} / \mathrm{min}(0.20$ to $0.25 \mathrm{in} / \mathrm{min}$ ) and allow the machine to run at this speed until the specimen breaks. This may be done only with relatively ductile materials and on a machine with a weighing system with response rapid enough to produce accurate results.

\section{Calculation}

11.1 Compressive Strength-Calculate the compressive strength by dividing the maximum compressive load carried by the specimen during the test by the original minimum cross-sectional area of the specimen. Express the result in megapascals or pounds-force per square inch and report to three significant figures.

11.2 Compressive Yield Strength-Calculate the compres sive yield strength by dividing the load carried by the specimen at the yield point by the original minimum cross-sectional area of the specimen. Express the result in megapascals or pounds-force per square inch and report to three significant figures.

11.3 Offset Yield Strength-Calculate the offset yield streagth by the method referred to in 4.10.

11.4 Modulus of Elasticity-Calculate the modulus of elasticity by drawing a tangent to the initial linear portion of the load deformation curve, selecting any point on this straight line portion, and dividing the compressive stress represented by this point by the corresponding strain, meas ure from the point where the extended tangent line intersects the strain-axis. Expross the result in gigapascals or poundsforce per square inch and report to three significant figures (See Annex A1).

11.5 For each series of tests, calculate to three significant figures the arithmetic mean of all values obtained and report as the "average value" for the particular property in question.

11.6 Calculate the standard deviation (estimated) as follows and report to two significant figures.

TABLE 1 Prucision, Compreschve Strungth (Values are in Units of Megapascats)

\begin{tabular}{|c|c|c|c|c|c|}
\hline Materid & Averge & $s_{p}^{A}$ & $s_{n}=$ & $r$ & $R^{\circ}$ \\
\hline $\begin{array}{l}\text { Acets } \\
\text { Polystyrion } \\
\text { Linen Filied Phenclic }\end{array}$ & $\begin{array}{l}100 \\
100 \\
158\end{array}$ & $\begin{array}{l}1.1 \\
1.4 \\
3.7\end{array}$ & $\begin{array}{l}2.1 \\
3.5 \\
7.5\end{array}$ & $\begin{array}{r}3.1 \\
3.9 \\
10.4\end{array}$ & $\begin{array}{r}5.9 \\
9.8 \\
21.0\end{array}$ \\
\hline
\end{tabular}

$A s$, is the mothin-aboratory standerd devation of the average.

- $S_{n}$ is the betmean-leboratory standerd deviation of the everege.

$c r=2.8 \mathrm{~S}$

$0 R=2.8 s_{n}$
TABLE 2 Prection, Compressive Modulus (Vatues as in Units of Megapascals)

\begin{tabular}{|c|c|c|c|c|c|}
\hline Mrtents & Averge & $S_{i}^{A}$ & $S_{n}^{*}$ & $r^{c}$ & $A^{\circ}$ \\
\hline $\begin{array}{l}\text { Actit } \\
\text { Paystyrum } \\
\text { Linen Find Phonolic }\end{array}$ & $\begin{array}{l}3.28 \\
3.88 \\
6.82\end{array}$ & $\begin{array}{l}0.14 \\
0.07 \\
0.23\end{array}$ & $\begin{array}{l}0.25 \\
0.74 \\
0.90\end{array}$ & $\begin{array}{l}0.39 \\
0.20 \\
0.84\end{array}$ & $\begin{array}{l}0.70 \\
2.07 \\
2.52\end{array}$ \\
\hline
\end{tabular}

$A s$, is the withinkeboratory stenderd deviation of the everege.

- $S_{n}$ is the bermeandaboratory stendard deviation of the averege.

$c r=2.8 s_{p}$

$0 \quad R=2.8 S_{\text {, }}$

$$
s=\sqrt{\left(\Sigma X^{2}-n X^{2}\right) /(n-1)}
$$

where:

$s=$ estimated standard deviation,

$X=$ value of single observation,

$n=$ number of observations, and

$\bar{X}=$ arithmetic mean of the set of observations.

Norr 11-Tbe method for determining the offset compressive yield strength is sirilar to that described in the Annex of Ter Method D 638.

\section{Report}

12.1 Report the following information:

12.1.1 Complete identification of the material tested, including type, source, manufacturer's code number, form, principal dimensions, previous history, etc.,

12.1.2 Method of preparing test specimens,

12.1.3 Type of test specimen and dimensions,

12.1.4 Conditioning procedure used.

12.1.5 Atmospheric conditions in test room,

12.1.6 Number of specimens tested,

12.1.7 Speed of testing,

12.1.8 Compressive strength, average value, and standard deviation

12.1.9 Compressive yield strength and offset yield strength average value, and standard deviation, when of interest,

12.1.10 Modulus of elasticity in compression (if required), average value, standard deviation, and

\subsubsection{Date of test.}

\section{Precision and Bias?}

13.1 Tables 1 and 2 are based on a round robin conducted in 1987 per Practice E 691, involving three materials tested by six laboratories for D 695M. Since the test parameters overiap within tolerances and the test values are normalized. the same data is used for both methods. For each material all of the samples were prepared at one source. Each ter result was the average of five individual determinations Each lab obtained two test results for each material.

NoTE 12-Cantion The following explanations of $r$ and $R$ (13: through 13.2.3) are only intended to present a meaningful way a considering the approximate precision of this tes method. The data is Tables 1 and 2 should not be rigorousty applied to aceeptance $\alpha$ rejection of material, as those darn are specific to the round robin at may not be representative of otber lots condivions materials a laboratories. Users of this tes method should apply the principles outlined in Practice E 691 to generate data specific to their laborator? and materials or berwen specific laboratories. The principles of 13: through 13.2.3 would then $b$ ve valid for such data.

13.2 Concept of $r$ and $R$-If $S(r)$ and $S(R)$ have beto

'Supporing dan are available from ASTM Headquarers. Reques Re D-20-11s0. 
alculated from a large enough body of data, and for test results that were averages from testing five specimens:

13.2.1 Repeatability, r-Comparing two test results for the same material, obtained by the same operator using the same equipment on the same day. The two test results should be judged not equivalent if they differ by more than the $r$ value for that material.

13.2.2 Reproducibility, $R$-Comparing two results for the same material, obtained by different operators using different equipment on different days. The two test resuits should be judged not equivalent if they differ by more than $R$ value for that material.

13.2.3 Any judgement per 13.2.1 and 13.2.2 would bave an approximate $95 \%(0.95)$ probability of being correct.

13.3 There are no recognized standards by which to estimate bias of this test method.

\section{ANNEX}

\section{(Mandatory Information)}

\section{A1. TOE COMPENSATION}

Al.1 In a typical stress-strain curve (Fig A1.1) there is a toe region, $A C$, that does not represent a property of the material. It is an artifact caused by a takeup of siack, and alignment or seating of the specimen. In order to obtain correct values of such parameters as modulus, strain, and offset yield point, this artifact must be compensated for to give the corrected zero point on the strain or extension axis.

A1.2 In the case of a material exhibiting a region of Hookean (linear) behavior (Fig. A1.1), a continuation of the linear $(C D)$ region of the curve is constructed through the zero-stress axis. This intersection $(B)$ is the corrected zerostrain point from which all extensions or strains must be measured, including the yield offset $(B E)$, if applicable. The elastic modulus can be determined by dividing the stress at

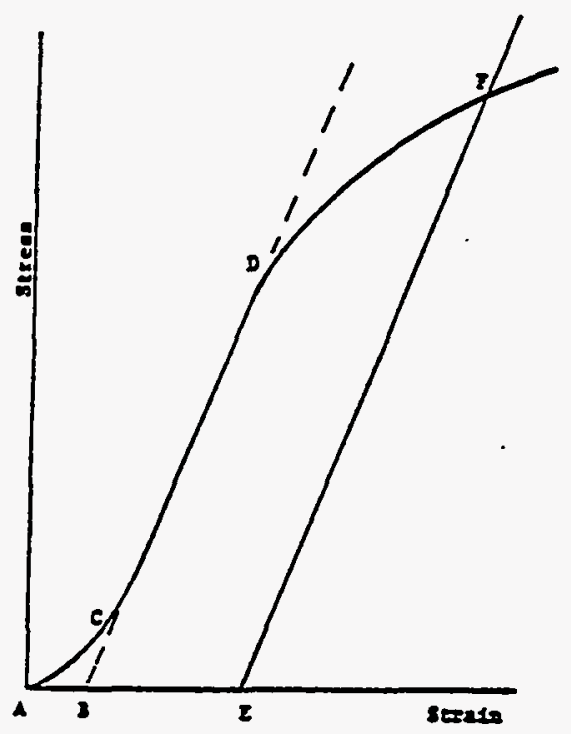

Note-Some chart recorders plot the mitror insog of this graph. FIG. A1.1 Material with Hookean Region any point along the line $C D$ (or its extension) by the strain at the same point (measured from point $B$, defined as zerostrain).

A1.3 In the case of a material that does not exhibit any linear region (Fig. A1.2), the same kind of toe correction of the zero-strain point can be made by constructing a tangent to the maximum slope at the inflection point $\left(H^{\prime}\right)$. This is extended to intersect the strain axis at point $B^{\prime}$, the corrected zero-strain point. Using point $B^{\prime}$ as zero strain, the stress at any point $\left(G^{\prime}\right)$ on the curve can be divided by the strain at that point to obtain a secant modulus (slope of line $B^{\prime} G^{\prime}$ ). For those materials with no linear region, any atrempt to use the tangent through the inflection point as a basis for determination of an offset yield point may result in unacceptable error.

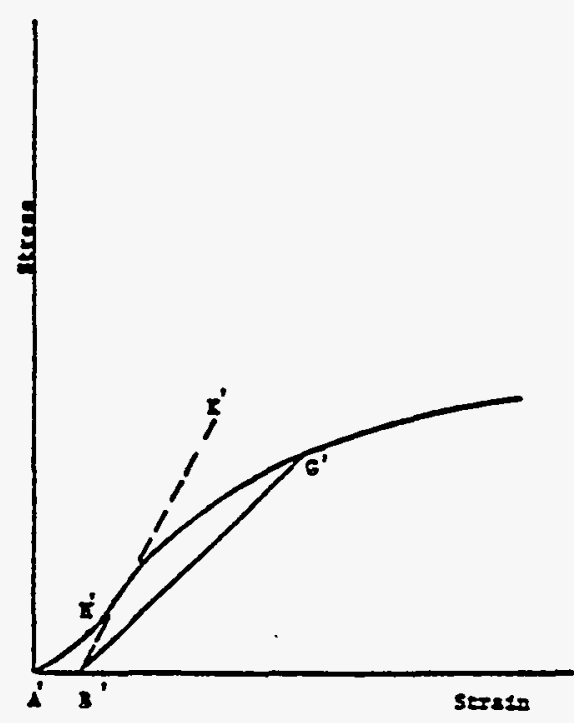

Nore-Scme chen recorders plot the mirrer insops of this graph. FG. A12 Material with No Hookean Region 


\section{佣 D 695}

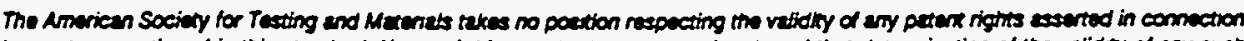

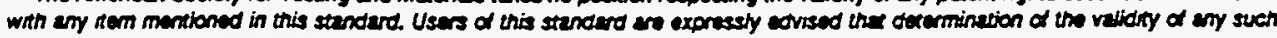

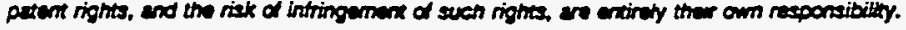

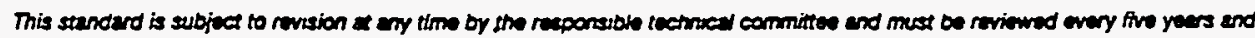

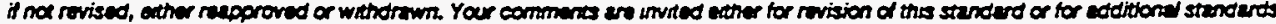

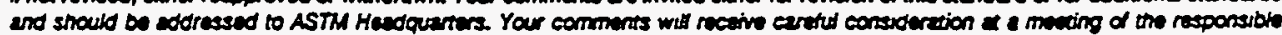

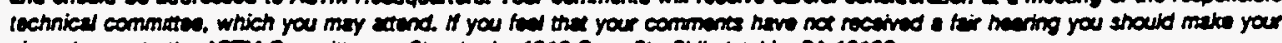

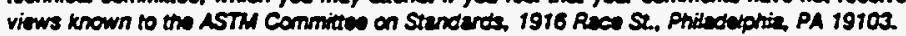




\title{
Standard Test Methods for Specific Gravity (Relativs Density) and Density of Plastics by Displacement'
}

\begin{abstract}
This standard is issued under the fixed designation D 792; the number immediately following the designation indicaies the year of original adoption or, in the case of revision, the year of hat revision. A number in parentheses indicates the year of hast reapproval. A superscript epsilon (e) indicates in editorial change since the last revision or reapproval.
\end{abstract}

These lest methods have been approved for use by asencies of the Department of Defense to replace method 5011 and 5012 of Federal Test Method Standard 106 and 14011 and 14021 of FTMS 601 . and for listing in the DoD Index of Specifications and Standards.

\section{Scope}

1.1 These test methods describe the determination of the specific gravity (relative density) and density of solid plastics in forms such as sheets, rods, tubes, or molded items.

1.2 Two test methods are described:

1.2.1 Test Method $A$-For testing solid plastics in water, and

1.2.2 Test Method B-For testing solid plastics in liquids other than water.

NoTE 1-Alternatively, Test Method D 1505 may be applied to many such forms, as well as to films and sheeting.

1.3 The values stated in SI units are to be regarded as the standard.

1.4 This standard may involve hazardous materials, operations, and equipment. This standard does not purport $t 0$ address all of the safety problems associated with its use. It is the responsibility of the user of this standard to establish appropriate safety and health practices and determine the applicability of regulatory limitations prior to use.

\section{Referenced Documents}

2.1 ASTM Standards:

D618 Methods of Conditioning Plastics and Electrical Insulating Materials for Testing ${ }^{2}$

D891 Test Methods for Specific Gravity of Liquid Industrial Chemicals ${ }^{3}$

D 1505 Test Method for Density of Plastics by the Density-Gradient Technique

D 1622 Test Method for Apparent Density of Rigid Cellular Plastics ${ }^{5}$

D 1898 Practice for Sampling of Plastics ${ }^{5}$

E 1 Specification for ASTM Thermometers ${ }^{6}$

E 12 Definitions of Terms Relating to Density and Specific Gravity of Solids, Liquids, and Gases?

\footnotetext{
1 These test methods are under the jurisdiction of ASTM Committee D-20 on Plastics and are the direct rexponsibility of Subcommitte D20.70 on Analytical Methods (Section D20.70.01)

Current edition approved May 30, 1986. Publisbed July 1986. Originally published as D 792 - 44. Lest previous edition D 792 - $66(1979)^{\ell t}$.

2 Annual Book of ASTM Siandards, Vols 08.01, 10.01, and 10.02

3 Annual Book of ASTM Slandards, Vol 15.05.

4 Annual Book of ASTM Standards, Vol 08.01.

s Annual Book of ASTM Siandards, Vol 08.02

Annual Book of ASTM Siandards, Vols 05.03 and 14.03 .

Annual Book of ASTM Siandards, Vols 04.02 and 15.05 .
}

E 691 Practice for Conducting an Interlaboratory Test Program to Determine the Precision of Test Methods ${ }^{8}$

\section{Definitions}

3.1 specific gravity (relative density)-the ratio of the weight in air of a unit volume of the impermeable portion of the material at $23^{\circ} \mathrm{C}\left(73.4^{\circ} \mathrm{F}\right)$ to the weight in air of equal density of an equal volume of gas-free distilled water at the same temperature. The form of expression shall be:

$$
\begin{aligned}
& \text { Specific gravity (relative density) } 23 / 23^{\circ} \mathrm{C} \\
& \text { (or sp gr } 23 / 23^{\circ} \mathrm{C} \text { ) }
\end{aligned}
$$

NoTe 2-This definition is essentially equivalent to the definition for apparent specific gravity and apparent density in Definitions E 12, because the small percentage difference introduced by not correcting for the buoyancy of air is insignificant for most purposes.

3.2 density - the weight in air in milligrams per cubic metre of impermeable portion of the material at $23^{\circ} \mathrm{C}$. The form of expression shall be:

$$
D^{23}, \mathrm{mg} / \mathrm{m}^{3} \text { (Notes 2, 3, 4) }
$$

NoTe 3-Densities in other units may be obtained, when desired, by multiplying by appropriate factors.

Note $4-S$ pecific gravity $23 / 23^{\circ} \mathrm{C}$ can be converted to density $23^{\circ} \mathrm{C}$ $\mathrm{mg} / \mathrm{m}^{3}$, by use of the following equation:

$$
\mathrm{D}^{23 \mathrm{C}}, \mathrm{mg} / \mathrm{m}^{3}=\mathrm{sp} \mathrm{gr} 23 / 23^{\circ} \mathrm{C} \times 0.9975
$$

\section{Summary of Test Methods}

4.1 A specimen of the solid plastic is weighed in air. It is then immersed in a liquid, its loss in weight upon immersion is determined, and its specific gravity (relative density) calculated.

\section{Significance and Use}

5.1 The specific gravity or density of a solid is a property that can be measured conveniently to identify a material, to follow physical changes in a sample, to indicate degree of uniformity among different sampling units or specimens, or to indicate the average density of a large item.

5.2 Changes in density of a single specimen may be due to changes in crystallinity, loss of plasticizer, absorption of solvent, or to other causes. Portions of a sample may differ in density because of difference in crystallinity, thermal history, porosity, and composition (types or proportions of resin, plasticizer, pigment, or filler).

Note 5-Reference is made to Test Method D 1622.

\footnotetext{
Anmual Book of ASTM Standards, Vols 06.03, 08.03, and 14.02
} 
5.3 Density is useful for calculating strength-weight and cost-weight ratios.

\section{Sampling}

6.1 The sampling units used for the determination of specific gravity (relative density) shall be representative of the quantity of product for which the data are required, in accordance with Practice D 1898.

6.1.1 If it is known or suspected that the sample consists of two or more layers or sections having different specific gravities, either complete finished parts or complete cross sections of the parts or shapes shall be used as the specimens, or separate specimens shall be taken and tested from each layer. The specific gravity (relative density) of the total part cannot be obtained by adding the specific gravity of the layers, unless relative percentages of the layers are taken into account.

\section{Conditioning}

7.1 Conditioning-Condition the test specimens at $23 \pm$ $2^{\circ} \mathrm{C}\left(73.4 \pm 3.6^{\circ} \mathrm{F}\right)$ and $50 \pm 5 \%$ relative humidity for not less than $40 \mathrm{~h}$ prior to test in accordance with Procedure $A$ of Methods D 618, for those tests where conditioning is required. In cases of disagreement, the tolerances shall be $1^{\circ} \mathrm{C}$ $\left(1.8^{\circ} \mathrm{F}\right)$ and $\pm 2 \%$ relative humidity.

7.2 Test Conditions-Conduct tests in the Standard Laboratory Atmosphere of $23 \pm 2{ }^{\circ} \mathrm{C}\left(73.4 \pm 3.6^{\circ} \mathrm{F}\right)$ and $50 \pm 5 \%$ relative humidity, unless otherwise specified in the test methods or in this specification. In cases of disagreement, the tolerances shall be $1^{\circ} \mathrm{C}\left(1.8^{\circ} \mathrm{F}\right)$ and $\pm 2 \%$ relative humidity.

\section{TEST METHOD A FOR TESTING SOLID PLASTICS IN WATER (SPECIMENS 1 TO $50 \mathrm{~g}$ )}

\section{Scope}

8.1 This test method involves weighing a one-piece specimen of 1 to $50 \mathrm{~g}$ in water, using a sinker with plastics that are lighter than water. This test method is suitable for plastics that are wet by, but otherwise not affected by water.

\section{Apparatus}

9.1 Analytical Balance-A balance with a precision within $0.1 \mathrm{mg}$ accuracy within $0.05 \%$ relative (that is, $0.05 \%$ of the weight of the specimen in air), and equipped with a stationary support for the immersion vessel above the balance pan ("pan straddle").

Nore 6-Assurance that the balance meets the performance requirements should be provided by frequent checks on adjustments of zero point and sensitivity and by periodic calibration for absolute accuracy, using standard weights.

9.2 Wire-A corrosion-resistant wire for suspending the specimen.

9.3 Sinker-A sinker for use with specimens of plastics that have specific gravities less than 1.000 . The sinker shall: (1) be corrosion-resistant; (2) have a specific gravity of not less than $7.0 ;(3)$ have smooth surfaces and a regular shape; and (4) be slightly heavier than necessary to sink the specimen. The sinker should have an opening to facilitate attachment to the specimen and wire.

9.4 Immersion Vessel-A beaker or other wide-mouthed vessel for holding the water and immersed.specimen.
9.5 Thermometer-A thermometer with an accuracy of $\pm 1^{\circ} \mathrm{C}\left( \pm 2^{\circ} \mathrm{F}\right)$ is required if the test is not performed in the Standard Laboratory Atmosphere of Methods D 618, (compare 16.4).

\section{Materials}

10.1 Water-The water shall be substantially air-free, distilled, or demineralized water.

NoTE 7-Water may be rendered substantially air-free by boiling and cooling or by shaking under vacuum in a heavy-walled vacuum flask (Precaution: Use gloves and shielding.) If the water does not wet the specimen, a few drops of a wetting agent shall be added. If this solution does not wet the specimen, Method B shall be used.

\section{Test Specimens}

11.1 The test specimen shall be a single piece of the material under test of any size and shape that can conve. niently be prepared and tested, provided that its volume shall be not less than $1 \mathrm{~cm}^{3}\left(0.06\right.$ in. $\left.^{3}\right)$, and its surface and edges shall be made smooth. The thickness of the specimen should be at least $1 \mathrm{~mm}(0.04$ in.) for each $1 \mathrm{~g}$ of weight. A specimen weighing 1 to $5 \mathrm{~g}$ usually will be found convenient, but specimens up to approximately $50 \mathrm{~g}$ may be used (Note 8). Care should be taken in cutting specimens to avoid changes in density resulting from compressive stresses or frictional heating.

NOTE 8-Specifications for certain plastics require a particular method of specimen preparation and should be consulted if applicable

11.2 The specimen shall be free from oil, grease, and other foreign matter.

\section{Procedure}

12.1 Weigh the specimen in air to the nearest $0.1 \mathrm{mg}$ for specimens weighing 1 to $10 \mathrm{~g}$ or to the nearest $\mathrm{mg}$ for specimens weighing more than 10 to $50 \mathrm{~g}$.

12.2 Attach to the balance a piece of fine wire sufficiently long to reach from the hook above the pan to the support for the immersion vessel. Attach the specimen to the wire such that it is suspended about $2.5 \mathrm{~cm}$ (1 in.) above the vessel support.

NoTE 9-The specimen may be weighed in air after hanging from the wire. In this case, record the weight of the specimen, $a=$ (weight of specimen + wire, in air) - (weight of wire in air).

12.3 Mount the immersion vessel on the support, and completely immerse the suspended specimen (and sinkers, if used) in water (10.1) at a temperature of $23 \pm 2^{\circ} \mathrm{C}$. The vessel must not touch wire or specimen. Remove any bubbles adhering to the specimen, wire, or sinker, paying particular attention to holes in the specimen and sinker. Usually these bubbles can be removed by rubbing them with another wire. If the bubbles cannot be removed by this method or if bubbles are continuously formed (as from dissolved gases), the use of vacuum is recommended (Note 11). Weigh the suspended specimen to the required precision (12.1) (Note 10). Record this weight as $b$ (the weight of the specimen, sinker, if used, and the partially immersed wire in liquid). Unless otherwise specified, weigh rapidly in order to minimize absorption of water by the specimen.

NoTe 10-It may be necessary to change the sensitivity adjustment of the balance to overcome the damping effect of the immersed specimen. 
NoTE 11-Some specimens may contain absorbed or dissolved gases, a irregularities which tend to trap air bubbles; any of these may affect the density values obtained. In such cases, the immersed specimen may be subjected to vacuum in a separate vessel until evolution of bubbles bes substantially ceased before weighing (see Test Method B). It must iso be demonstrated that the use of this technique leads to results of the required degree of precision.

12.4 Weigh the wire (and sinker, if used) in water with immersion to the same depth as used in the previous step (Notes 12 and 13). Record this height as $w$ (weight of the wire in liquid).

NOTE 12-It is convenient to mark the level of immersion by means of a shallow notch filed in the wire. The finer the wire, the greater the wlerance which may be permitted in adjusting the level of immersion between weighings. With wire Awg No. 36 or finer, disregard its degrees of immersion and, if no sinker is used, use the weight of the wire in air $25 w$.

Note 13-If the wire is left attached to the balance arm during a series of determinations, the weight $a$ may be determined either with the id of a tare on the other arm of the balance or as in Note 11. In such eases, care must be taken that the change of weight of the wire (for example, from visible water) between readings does not exceed the desired precision.

12.5 Repeat the procedure for the required number of specimens. Two specimens per sample are recommended. Determine acceptability of number of replicate test specimens by comparing results with precision data given in Tables 1 and 2 of Section 23. Additional specimens may be required to give the desired precision.

\section{Calculation}

13.1 Calculate the specific gravity of the plastic as follows:

$$
\text { Sp gr } 23 / 23^{\circ} \mathrm{C}=a /(a+w-b)
$$

where:

$a=$ apparent weight of specimen, without wire or sinker, in air,

$b=$ apparent weight of specimen (and of sinker, if used) completely immersed and of the wire partially immersed in liquid, and

$w=$ apparent weight of totally immersed sinker (if used) and of partially immersed wire.

13.2 Calculate the density of the plastic as follows:

$$
\mathrm{D}^{23 \mathrm{C}}, \mathrm{mg} / \mathrm{m}^{3}=\mathrm{sp} \mathrm{gr} 23 / 23^{\circ} \mathrm{C} \times 0.9975
$$

\section{Report}

14.1 The report shall include the following:

14.1.1 Complete identification of the material or product tested, including method of specimen preparation and conditioning,

14.1.2 Average specific gravity (relative density) for all specimens from a sampling unit, reported as sp gr $23 / 23^{\circ} \mathrm{C}=$ or average density reported as $D^{23 C}=-\mathrm{g} / \mathrm{cm}^{3}$,

14.1.3 A measure of the degree of variation of specific gravity or density within the sampling unit such as the standard deviation and number of determinations on a homogeneous material or the averages plus these measures of dispersion on different layers or areas of a nonhomogeneous product,

14.1.4 Any evidence of porosity of the material or specimen,

14.1.5 The method of test (Method A of Methods D 792), and

14.1.6 Date of test.

15. Precision and Bias

15.1 See Section 23.

TEST METHOD B FOR TESTING SOLID PLASTICS IN LIQUIDS OTHER THAN WATER (SPECTMENS 1 TO $50 \mathrm{~g}$ )

\section{Scope}

16.1 Test Method B uses a liquid other than water for testing one-piece specimens, 1 to $50 \mathrm{~g}$, of plastics that are affected by water or which are lighter than water.

\section{Apparatus}

17.1 The apparatus shall include the balance, wire, and immersion vessel of Section 8, and, optionally, the following:

17.2 Pycnometer with Thermometer-A $25-\mathrm{mL}$ specific gravity bottle with thermometer, or

17.3 Pycnometer-A pycnometer of the Weld type, preferably with a capacity of about $25 \mathrm{~mL}$ and an external cap over the stopper.

17.4 Thermometer-A thermometer having not fewer than four divisions per ${ }^{\circ} \mathrm{C}$ or two divisions per ${ }^{\circ} \mathrm{F}$ over a temperature range of not less than $5^{\circ} \mathrm{C}$ or $10^{\circ} \mathrm{F}$ above and below the standard temperature, and having an ice point for calibration. A thermometer short enough to be handled inside the balance case will be found convenient. ASTM Thermometer 23C (see Specification E 1) and Anschütz-type thermometers have been found satisfactory for this purpose.

17.5 Constant-Temperature Bath-An appropriate constant-temperature bath adjusted to maintain a temperature of $23 \pm 0.1^{\circ} \mathrm{C}$.

\section{Materials}

18.1 Immersion Liquid-The liquid used shall not dissolve, swell, or otherwise affect the specimen, but should wet it and should have a specific gravity less than that of the specimen. In addition, the immersion liquid should be nonhygroscopic, have a low vapor pressure, a low viscosity, and a high flash point, and should leave little or no waxy or tarry residue on evaporation. A narrow cut distilled from kerosine meets these requirements for many plastics. The specific gravity $23 / 23^{\circ} \mathrm{C}$ of the immersion liquid shall be determined shortly before and after each use in this method to a precision of at least $0.1 \%$ relative, unless it has been

TABLE 1 Tost Mothod A Tested in Water

\begin{tabular}{llllll}
\hline Material & Mean & $S_{r}$ & $S_{n}$ & $I_{A}$ \\
\hline Polypropylene & 0.9007 & 0.00196 & 0.00297 & 0.00555 \\
Cellulose Acetate Butyrate & 1.1973 & 0.00232 & 0.00304 & 0.00657 \\
Potyphenylene Sulfide & 1.1708 & 0.00540 & 0.00738 & 0.00860 \\
Thermoset & 1.3136 & 0.00271 & 0.00313 & 0.02089 \\
Potyvinyl Chiloride & 1.3396 & 0.00243 & 0.00615 & 0.02171 & 0.00688 \\
\hline
\end{tabular}


TABLE 2 Teat Method B Teated in Liquids Other Than Water

\begin{tabular}{|c|c|c|c|c|c|}
\hline Materla & Moan & $S_{f}$ & $S_{A}$ & I & In \\
\hline $\begin{array}{l}\text { Potypropylene } \\
\text { LDPE } \\
\text { HDPE } \\
\text { Thermoset }\end{array}$ & $\begin{array}{l}0.9023 \\
0.9215 \\
0.9878 \\
1.3130\end{array}$ & $\begin{array}{l}0.00139 \\
0.00109 \\
0.00126 \\
0.00160\end{array}$ & $\begin{array}{l}0.00239 \\
0.00195 \\
0.00189 \\
0.00217\end{array}$ & $\begin{array}{l}0.00393 \\
0.00308 \\
0.00356 \\
0.00453\end{array}$ & $\begin{array}{l}0.00678 \\
0.00552 \\
0.01007 \\
0.01282\end{array}$ \\
\hline
\end{tabular}

established experimentally in the particular application that a lesser frequency of determination can be used to assure the desired precision.

NoTE 14-For the determination of the specific gravity of the liquid, the use of a standard plummet of known volume (Note 15) or of Method A, C, or D of Test Methods D 891, using the modifications required to give specific gravity $23 / 23^{\circ} \mathrm{C}$ instead of specific gravity $60 / 60^{\circ} \mathrm{F}$, is recommended. One suggested procedure is the following:

If a constant-temperature water bath is not available, determine the weight of the clean, dry pycnometer with thermometer to the nearest 0.1 mg on an analytical balance. Fill the pycnometer with water (10.1) cooler than $23^{\circ} \mathrm{C}$. Insert the thermometer-stopper, causing excess water to be expelied through the side arm. Permit the filled bottle to warm in air until the thermometer reads $23.0^{\circ} \mathrm{C}$. Remove the drop of water at the tip of the side arm with a bit of filter paper, taking care not to draw any liquid from within the capillary, place the cap over the side arm, wipe the outside carefully, and weigh the filled bottle again to the nearest 0.2 me. Empty the pycnometer, dry, and fill and weigh with the other liquid in the same manner as was done with the water. Calculate the specific gravity $23 / 23^{\circ} \mathrm{C}$ of the liquid, $d$, as follows:

where:

$$
d=(b-e) /(w-e)
$$

$e=$ apparent weight of empty pycnometer,

$w=$ apparent weight of pycnometer filled with water at $23.0^{\circ} \mathrm{C}$, and

$b=$ apparent weight of pycnometer filled with liquid at $23.0^{\circ} \mathrm{C}$.

If a constant-temperature water bath is available, a pycnometer without a thermometer may be used (compare 30.2).

NOTE 15-One standard object which has been found satisfactory for this purpose is the Reimann Thermometer Plummet. These are normally supplied calibrated for measurements at temperatures other than $23 / 23^{\circ} \mathrm{C}$, so that recalibration is necessary for the purposes of these methods.

\section{Test Specimens}

\subsection{See Section 11.}

\section{Procedure}

20.1 The procedure shall be similar to Section 12, except for the choice of immersion liquid, and the temperature during the immersed weighing (12.3) shall be $23 \pm 0.5^{\circ} \mathrm{C}$ $\left(73.4 \pm 1.0^{\circ} \mathrm{F}\right)$.

\section{Calculation}

21.1 The calculations shall be similar to Section 13, except that $d$, the specific gravity $23 / 23^{\circ} \mathrm{C}$ of the liquid, shall be placed in the numerator.

$$
\text { Sp gr } 23 / 23^{\circ} \mathrm{C}=(a \times d /(a+w-b)
$$

\section{Report}

\subsection{See Section 14.}

\section{Precision and Bias 9}

23.1 Tables 1 and 2 are based on round robins conducted in 1985 involving five materials tested by six laboratories for Test Method A. Four materials were tested with Test Method B by six laboratories. Each test result was based on two individual determinations. Each laboratory obtained four test results for each material.

23.2 In Tables 1 and 2, for the materials indicated, and for mean values that are derived from testing two specimens:

23.2.1 S is the within-laboratory standard deviation of the mean and $I_{r}=2.83 \mathrm{~S}_{r}$ (See 23.2.3 for application of $\mathrm{I}_{r}$ )

23.2.2 $\mathrm{S}_{R}$ is the between-laboratory standard deviation of the mean, and $\mathrm{I}_{R}=2.83 \mathrm{~S}_{R}$. (See 23.2.4 for application of $\mathrm{I}_{\mathrm{R}}$ )

23.2.3 Repeatability-In comparing two mean values for the same material obtained by the same operator using the same equipment on the same day, the means should be judged not equivalent if they differ by more than the $I$, value for that material and condition.

23.2.4 Reproducibility -In comparing two mean values for the same material obtained by different operators using different equipment on different days, the means should be judged not equivalent if they differ by more than the $\mathrm{I}_{R}$ value for that material and condition. (This applies between different laboratories or between different equipment within the same laboratory.)

23.2.5 The judgements per 23.2.3 and 23.2.4 will have an approximate $95 \%(0.95)$ probability of being correct.

23.2.6 Other materials may give somewhat different results.

23.3 For further information on the methodology used in this section, see Practice E 691 .

'Supporting data are available from ASTM Headquarters Reques RR:D 20 - 1133 .

\footnotetext{
The American Sociecy for Teating and Materials takes no position respecting the validty of any patent rights asserted in connection with any item mentioned in this standard. Users of this standard are expressly advised that determination of the valldity of any such peterx nighte, and the riste of infingement of such rights, are entrey thatr awn rosponsibility.
}

This standard is subject to reviliton ar any time by the responsiblo technical committes and must be reviowed overy five yoars and If not revised, either respoproved or withdrawn. Your comments are invited either for roviston of this standard or for additional stendards and should be addressed to ASTM Heedquarters. Your comments will recelve caroful consideration at a mooting of the responsible technieal committee, which you may attend. If you foel that your comments havo not roceived a fair hearing you should make your views known to the ASTM Committe on Standerds, 1916 Race St. Philadeiphla, PA 19103 


\section{Standard Test Method for Strength Properties of Adhesives in Shear by Tension Loading (Metal-to-Metal) ${ }^{1}$}

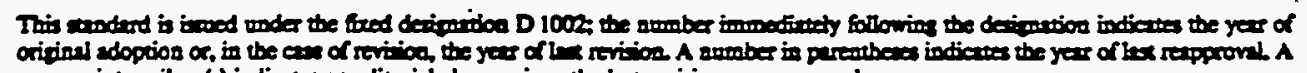

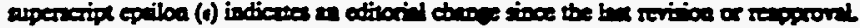

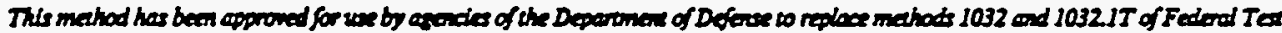

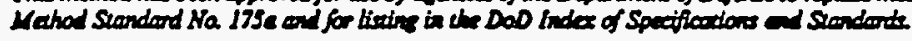

The accuracy of the results of strength tents of adhesive bonds will depend on the conditions under which the bonding process is carried out. Unless otherwise agreed upon by the manufacturer and the purchaser, the bondins conditions shall be preseribed by the manufacturer of the adhesive. In order to ensure that complete information is available to the individual conducting the tests, the manufacturer of the adhesive shall furnish numeriel values and other specific information for each of the following variables

(l) Procedure for preparation of surfaces prior to application of the adhesive, the cleaning and drying of metal surfaces, and specinl surface treatments such as sanding that are not specifically limited by the pertinent teat method.

(2). Complete mixing directions for the adherive

(3) Conditions for application of the adhesive, including the rate of spread or thickness of film, number of coats to be applied, whether to be applied to one or both surfaces, and the conditions of drying where more than one cont is required.

(4) Assembly conditions before application of pressure, including the room temperature, relative humidity, length of time, and whether open or closed assembly is to be used.

(5) Curing conditions, including the amount of pressure to be applied, the length of time under pressure, method of applying pressure (pressure bag, press platens, etc), heat-up rate, and the temperature of the assembly when under pressure. It should be stated whether this temperature is that of the bondline or of the atmosphere at which the assembly is to be maintained.

(ด) Conditioning procedure before testing, unless a standard procedure is specified, including the length of time, temperatuire, and relative humidity.

A range may be prescribed for any variable by the manufacturer of the adhesive if it can be assumed by the test operator that any artitrarily chosen value within such a range or any combination of such values for several variables will be acceptable to both the manufacturer and the purabaser of the adivive.

\section{Scope}

1.1 This test method covers the cstermination of the comparative shear strengths of adhesives for bonding metals when tested on a standard specimen and under specified conditions of preparation and test.

12 This standard does not pupport to address all of the safaty problems, if any, associated with its use. It is the responsibility of whoever uses this standard to consult and establish appropriate safay and health practices and determine the applicability of regulatory limitations prior to use. $\because:$

\section{Apparatu.}

- 21 The testing machine shall conform to the requiromerits of ASTM Methods E4, Verification of Testing

'This tes method is under the jurixtiction of ASTM Comminten D.14 an Adherive and is the direct responsibility of Subcommitue D14.80 oe Metal Booding Adherive

Curreac etition epproved fuly 22, 1972 Pubtisbed Ocoub- 1972 Oripinilly

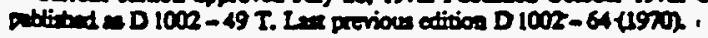

Machines. ${ }^{2}$ The texing machine shall be so selected that the breaking load of the specimens fills betreen 15 and 85 percent of the full-scale capacity. The machine shall be capable of maintaining a rate of loading of 80 to $100 \mathrm{~kg} / \mathrm{cm}^{2}$ (1200 to 1400 poi)/min, or, if the rate is dependent on crosshead motion, the machine should be set to approach this rate of loading It shall be provided with a suitable pair of self-aligning grips to bold the specimen. It is recommended that the jaws of these grips shall engage the outer $25 \mathrm{~mm}$ (1 in) of each ead of the test specimen firmily (Note 1). The grips and attachments shall be so constructed that they will move into alignment with the teat specimen as soon as the load is applied, so that the long axis of the test specimen will coincide with the direction of the applied pull through the center line of the grip assembly.

Norn 1-The length of overip of the specimen may be varied where neceseny. The length of the specimen in the jaws, bowever, must not be varied The distance from the end of the lap to the end of the jaws should be $63 \mathrm{~mm}(24 \mathrm{sin})$ in an test

\footnotetext{
2 Arual Book of ASTM Suadarts, Vol 0301.
} 


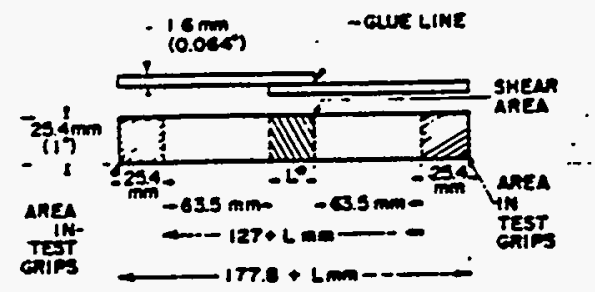

Fa. 1 Form and Dimasions of Text spectume

\section{Test Specimess}

3.1 Ter specimens shall conform to the form and dimensions shown in Fig. 1. These shall be cut from tert pariels prepared as prescribed in Section 4. The recommended thickness of the sheets is $1.62 \pm 0.125 \mathrm{~mm}(0.064 \pm 0.005$ in.). The recommended leagth of overlap for most metrils of $1.62 \mathrm{~mm}(0.064 \mathrm{in}$.) in thichess is $12.7 \pm 0.25 \mathrm{~mm}(0.5 \pm$ 0.01 in.).

Norr 2-Since it is underimble to exced the yield point of the meth in tension durios test the permivible leopth of overiap in the specimen will vary with the thickness and type of metal, and on the reseral level of strength of the adhesive beins investipted The muximum permivible leasth may be computed from the forlowios relationshipe

$$
L=F y t / s
$$

where

$L=$ leaph of overlep, in.

$l$ - thicines of metal, in.

Fity = yield point of meen (or the stren an propartional limit), pri, and

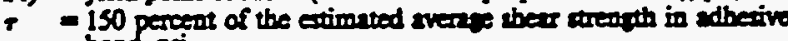
bond, pai.

NoTr 3-A veriation in thictors of the meth and the length of overap, will likety influence the tert values and make direcer comparison of dats quertionable For this resson, in comparrive or specification tests, the thicknes should preferibly be $1.62 \pm 0.125 \mathrm{~mm}(0.064 \pm$ 0.005 in.) and the leurth of ovedisp should perfently be $12.7 \pm 0.25$

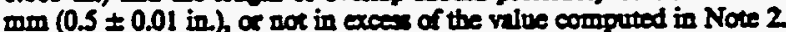
For development tests vilues could be different, but should then be constant.

3.2 The following grades of metal are recommended for the tex specimens

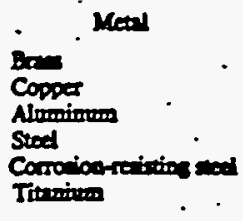

\begin{tabular}{|c|}
\hline ASTM Deciontion \\
\hline 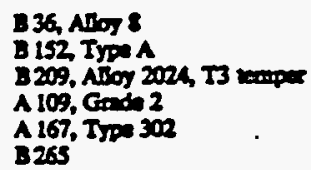 \\
\hline
\end{tabular}

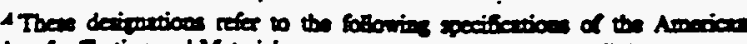
Socidy for Texine and Materite

B 36, for Bris Pinte, Stece, Strip and Roled Bur

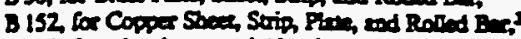

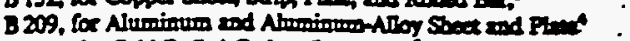

A 109. for Cold-Roled Curboa Sted String

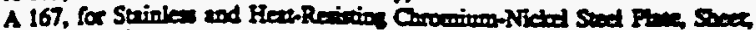
and Strip. and

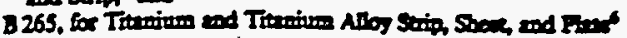

3 Anrual Book of ASTM Srandorte Val or oi

- Anual Book of ASTM Standard Vol 0202

i Anrual Book of ASTM Stondert, Vol 01.03

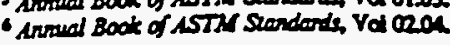

3.3 At least 30 specimens shall be tested, representing at least four different joints. However, if statistical analysis of data and variance is employed, it should be possible to reduce this number.

\section{Preparation of Teat Joints}

4.1 It is recommended that test specimens be made up in multiples of at least five specimens, and then cut into individual test specimens (Note 4), Fise, 2 and 3. Cut sheets of the metols prescribed in 3.1 and 3.2 to suitable size. Al edges of the metal parels and specimens which will be within (or which will bound) the lap joints shall be machined true (without burs or bevels and at right angles to faces) and smooth (ms 160 max) before the panels are surfece-treated and bonded. Clean and dry the sheets carefully, sccording to the procedure prescribed by the manufacturer of the adhesive, and assemble in pairs. Prepare and appiy the adhesive sccording to the recommendations of the manuiacturer of the adhesive. Apply the adhesive to a sufficient length in the area across the end of one or both metal sheets so that the adhesive will cover a space approximately $6 \mathrm{~mm}$ ( $y_{4}$ in.) longer than the overiap as selected in Section 3. Assernble the sheets so that they will be held rigidly so that the leagth of the overiap will be controlled, as indiated in Section 3, within $0.25 \mathrm{~mm}( \pm 0.01 \mathrm{in}$ ), and the adhesive allowed to cure as preseribed by the manufacturer of the adhesive.

Nors 4-Bonding specimens in multiple peocts is betieved to give mare representuive specimens Howeve, individual specimeos may be prepured if apcentie to the supplier or the purchiser of the adhesive.

\section{Preparation of Test Specimens}

5.1 Cut the tent specimens, as shown in Fis 1, from the panels, Figs, 2 and 3. Perform the cutting operation so as to avoid overheating or mechanical damage to the joints (Note 5). For final preparation trim panel area according to Fis 2 Messure the width of the specimen and the length of the overlap to the nearest $0.25 \mathrm{~mm}(0.01 \mathrm{in}$ ) to determine the shear area.

Norr S-A fivotooth typecter's circular saw hes been found suituble for such purpowe.

\section{Procedure}

6.1 Test the specimens, prepared as preseribed in Section $S$, as soon after preparation as possible. The manufacturer of the adhesive may, however, prescribe a definite period of conditioning under specific conditions before testing.

6.2 Place the specimens in the grips of the testing machine 30 that the outer $25 \mathrm{~mm}$ ( 1 in) of each end are in contact with the jaws (Note 1) and so that the long axis of the test specimen coincides with the direction of applied pull through the center line of the grip assembly. Apply the losding immediately to the specimen at the rate of 80 to $100 \mathrm{~kg} / \mathrm{cm}^{2}$ (1200 to 1400 poii) of the shear aren per min. Continue the load to failure. This rate of loading will be approximated by a free crosshead speed of $1.3 \mathrm{~mm}(0.05 \mathrm{in}$ )/min.

\section{Calcolitions}

7.1 Recond the load at failure and the nature and amount of this failure (cohesion in adhesive or metal, or adhesion) for each specimen Express all failing loads in kilograms pes 


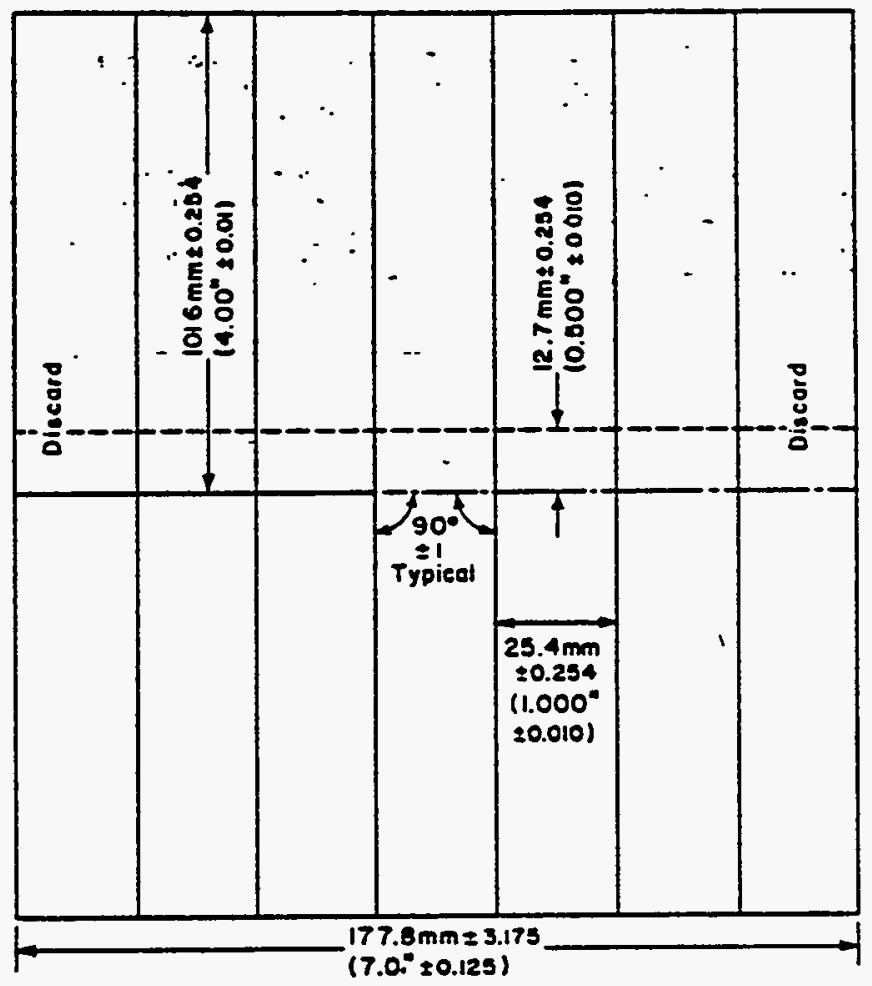

Fe 2 senderd Teet Pand

square centimeter (pounds per square inch) of shear area, elculated to the nearest $0.06 \mathrm{~cm}^{2}\left(0.01 \mathrm{in}^{2}\right)$.

\section{Report}

8.1 The report shall include the following information:

8.1.1 Complete identification of the adhesive tested, inctuding type, source, date manufactured, manufactures' code numbers, form, etc.,

8.1.2 Complete identification of the metal used, its thick. ness, and the method of cleaning and proparing its surface prior to bonding.

8.1.3 Application and bonding conditions used in pro paring specimeas,

8.1.4 Average thickness of adhesive layer after formation of the joint within 0.001 in. The method of obtaining the thickeness of the adhesive lnyer shall be described including procedure, location of measurements, and sange of measurements.

8.1.5 Length of overlap used,

8.1.6 Conditioning procedure used for specimens prior to testing.

8.1.7 Number of specimens tested

8.1.8 Number of joints represented and type of joint if other than single overlap,

8.1.9 Maximum, minimum, and average values for the failing load, and

8.1.10 The nature of the failure including the average estimated percentages of failure in the cohesion of the adhesive, contact failure, and adhesion to the metal.

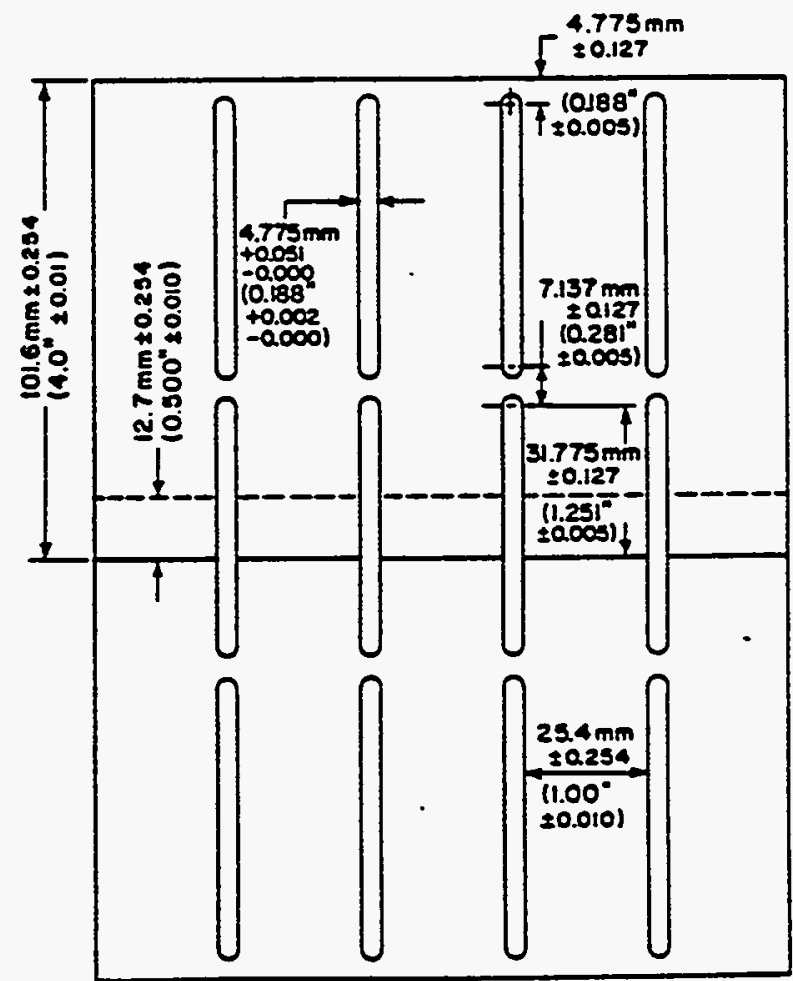

Fic. 3 Optond Pand for Acesptance Teats Onty 


\section{Ali $D 1002$}

$$
\ldots . .
$$

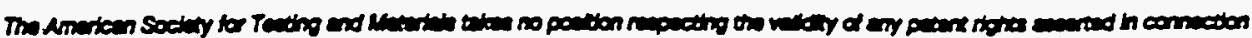

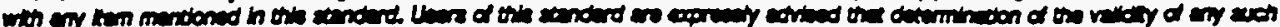

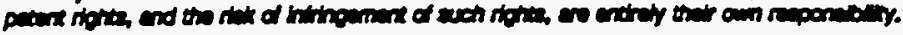

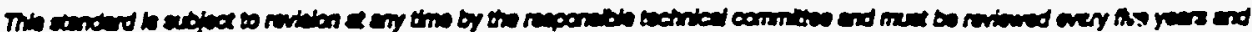

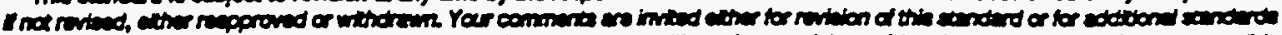

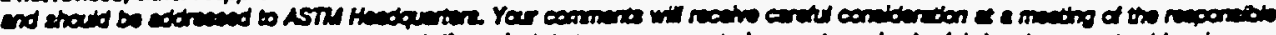

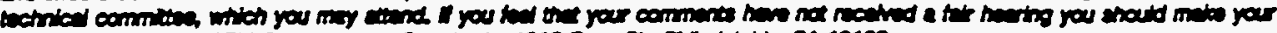

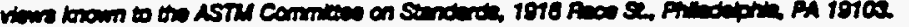




\title{
Standard Test Method for \\ Thermal Conductivity of Solids by Means of the Guarded- Comparative-Longitudinal Heat Flow Technique'
}

\begin{abstract}
This standard is issued under the fixed desination E 1225: the number immediately following the designation indiertes the jens of ongnal adoption or. in the case of revision, the yeat of less revision. A number in parentheses indielas the year of last reaproval. A superseripr epsilon (1) indieates an editorial change since the has revision or reapproval.
\end{abstract}

"NoTE-Editonal corrections were made throughoun in March 1990.

\section{Scope}

1.I This test method describes a steady state technique for the determination of the thermal conductivity, $\lambda$, of homogeneous-opaque solids (see Notes 1 and 2). This test method is for materials with effective conductivities in the approximate range $0.2<\lambda<200 \mathrm{~W} / \mathrm{m} \cdot \mathrm{K}$ over the approximate temperature range between 90 and $1300 \mathrm{~K}$. It can be used outside these ranges with decreased accuracy.

VOTE 1-For purposes of this technique 2 system is homogeneous if the apparent thermal conductivity of the specimen. $\lambda_{\lambda}$, does not vary with changes of thickness or cross-sectional area by more than $\pm 5 \%$. For composites or heterogeneous systems consisting of siabs or plates bonded together. the specimen should be more than 20 units wide and 20 units thick. respectively. where a unit is the thickness of the thickest slab or plate. so that diameter or length changes of one-half unit will affect the apparent $\lambda_{1}$ by less than $=5 \%$. For systems that are non-opaque or parially transparent in the infrared. the combined error due to inhomogeneity and photon unansmission should be less than \#5 c. Measurements on highly uransparent solids must be accompanied with infrared absorption coefficient information or the results must be reported as apparent $\lambda_{A}$.

SOTE 2-This test method may also be used to evaluate the thermal conductance/resistance of materials in contact.

1.2 This standard may involve hazardous materials, operations, and equipment. This standard does not purport 10 address all of the safety problems associated with its use. It is the responsibility of the user of this standard to establish appropriate safetr. and health practices and determine the applicability of regulaton limitarions prior to use.

\section{Referenced Documents}

\section{1 .AST.M Standards:}

C 177 Test Method for Steady State Heat Flux Measurements and Thermal Transmission Properties by Means of the Guarded-Hot-Plate Apparatus ${ }^{2}$

C 408 Test Method for Thermal Conductivity of Whiteware Ceramics ${ }^{3}$

C 1045 Practice for Calculating Thermal Transmission Properties from Steady State Heat Flux Measurements

I This test method is under the jurisdietion of ASTM Committe E.37 on Thermal Measurements and is the diren responsibility of Subcommmtte E37.01 on Test Miethods and Recommended Practices.

Current edition approved Dec. 31. 1987. Published February 1988.

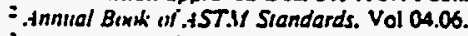

'. Innial Bemk of.tST.M Siandards. Vol 15.02.
D 4351 Test Method for Measuring the Thermal Co tivity of Plastics by the Evaporation-Caloris Method 4

E 230 Temperature-Electromotive Force (EMF) T for Standardized Thermocouples ${ }^{5}$

E 220 Method for Calioration of Thermocouple Comparison Techniques ${ }^{5}$

F 433 Practice for Evaluating Thermal Conciuctiv Gasket Materials ${ }^{6}$

3. Descriptions of Terms and Symbols Specific th Standard

\subsection{Terms:}

3.1.1 Thermal Conductivity, $\lambda$-In an optically thick with constant properties, the quantity of heat flow pe area at steady state, $q^{\prime}$, is given by $q^{\prime}=\lambda \nabla T$, where $\lambda$ thermal conductivity. For one-dimensional teat for $Z$ direction, this reduces to $q^{\prime}=\lambda \frac{d T}{d Z}$, where $d T / c$. temperature gradient. When only two seusors ire us differential must be replaced with $\Delta T / \Delta Z$, tive tempe: difference between the two sensors.

3.1.2 Apparent Thermal Conductivity-In some mens the quantity of heat flow will be a functi additional system parameters such as the eminanc bounding surfaces and the specimen size. In these case comparator determines only an apparent thermal $\infty$ tivity, $\lambda_{A}$, defined as $\lambda_{A}=-q^{\prime} \frac{\Delta Z}{\Delta T}$.

3.2 Symbols:

$\lambda_{M}(T) \quad=$ thermal conductivity of meter bars (reit materials) as a function of tempstanure. $m \cdot K)$.

$\lambda_{M}{ }^{3}=$ thermal conductivity of top meter ba $\boldsymbol{m} \cdot \mathbf{K})$,

$\lambda_{M}{ }^{2} \quad=$ thermal conductivity of bottom mete $(W / m \cdot K)$

$\lambda_{s}(T) \quad=$ thermal conductivity of specimen correct heat exchange where necessary, $(W / m \cdot k$

$\lambda^{\prime} s(T)=$ thermal conductivity of specimen calc. by ignoring heat exchange correction. $\boldsymbol{m} \cdot \mathrm{K})$,

\footnotetext{
Annual Book of ASTM Siandards. Vol 08.03

Annual Book of ASTM Siandards. Vol 14.03.

- Annual Book of AST.K Slandards. Vol 09.02
} 


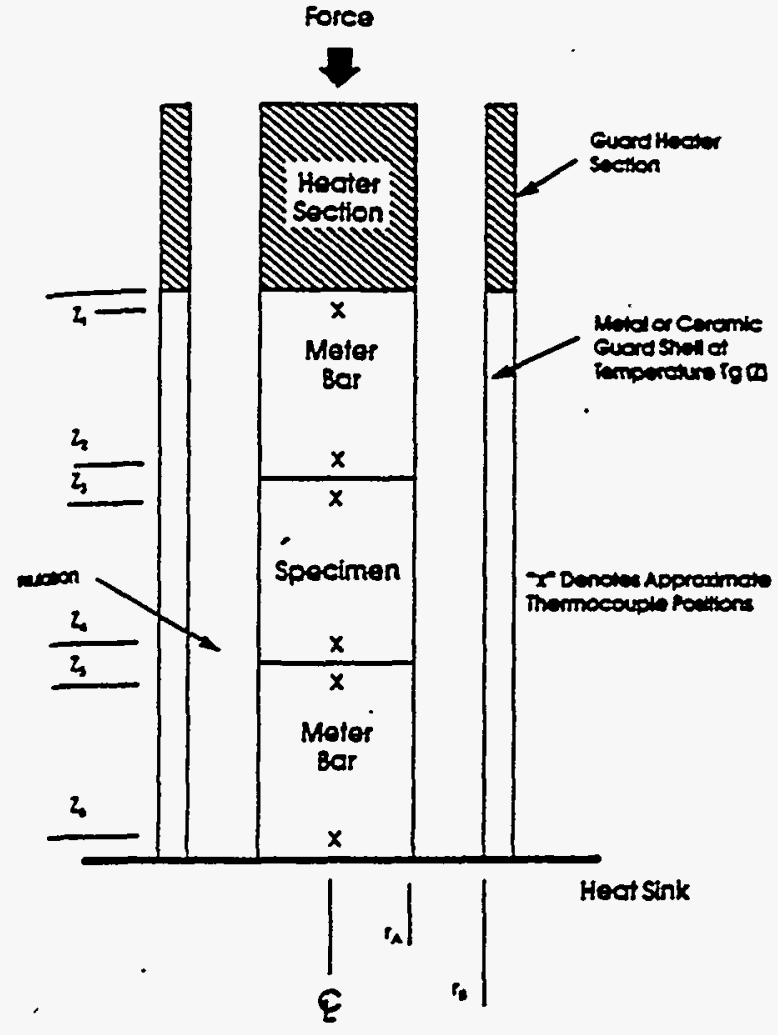

FG. 1(a) Schematic of a Comparative-Guarded-Longitudinal Mat flow System Showing Possible Locations of Temperature sensors

$4 n=$ thermal conductivity of insulation as a function of temperature, $(\mathrm{W} / \mathrm{m} \cdot \mathrm{K})$.

I = absolute temperature in degrees Keivin (K).

L $\quad=$ position as measured from the upper end of the column, $(m)$.

$i \quad=$ specimen length. $(m)$.

$T_{1} \quad=$ the temperature at $Z_{n}(\mathrm{~K})$.

$q^{\prime} \quad=$ heat flow per unit area $\left(W / m^{3}\right)$.

id. $\delta T$. etc. $=$ uncertainty in $\lambda, T$, etc.,

$r \quad=$ specimen radius, $(m)$.

$\Rightarrow$ guard cylinder inner radius, $(m)$.

$T(=) \quad=$ guard temperature as a function of position, $z$, (K), and

7. $\quad \Rightarrow$ distance from upper end of the column. (m).

4. Summary. of Test Method

4.1 A test specimen is inserted under load between two imilar specimens of a material of known thermal properties. I temperature gradient is established in the test stack and heat losses minimized by use of a longitudinal' guard having approximately the same temperature gradient conditions. At aquilibrium conditions. the thermal conductivity is derived in terms of the temperature gradients in the respective specimens and the thermal conductivity of the reference materials.

4.2 General Features of Test .1Yethod:

4.2.1 The general features of the guarded longitudinal

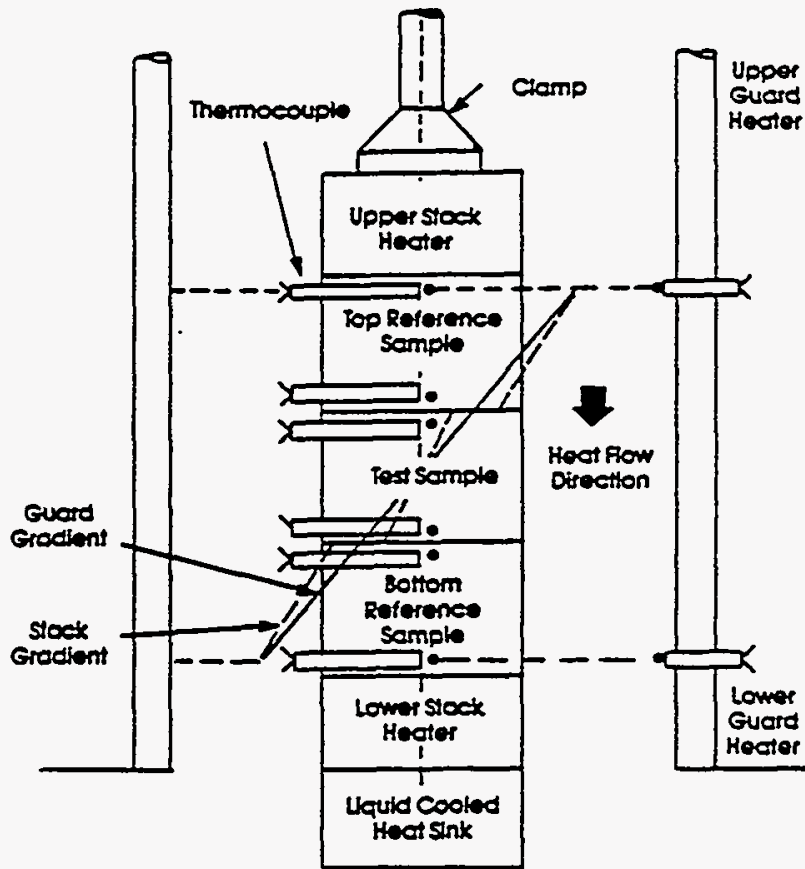

FIG. 1(b) Schematic of Typical Test Stack and Guard System Illustrating Matehing of Temperature Gradients

heat flow technique are shown in Fig. 1. A specimen of unknown conductivity. $\lambda_{s}$. but having an estimated thermal conductance of $\lambda_{s} / l_{s}$. is mounted between two meter bars of known thermal conductivity. $\lambda_{1 y}$ of the same cross-section and similar thermal conductance. $\lambda_{s g} / /_{. y}$. A more complex but suitable arrangement is a column consisting of a disk heater with a specimen and a meter bar on each side between heater and heat sink. Approximately one-half of the power would then flow through each specimen. When the meter bars and specimen are right-circular cylinders of equal diameter the technique is described as the cut-bar method. When the cross-sectional dimensions are larger than the thicknesss it is described as the flat slab comparative. Essentially any shape can be used as long as the meter bars and specimen have the same conduction areas.

4.2. A force is applied to the column that is surrounded by an insulation material of thermal conductivity, $\lambda_{1}$. The insulation is enclosed in a guard shell with a radius. $r_{B}$, and the shell is held at temperature. $T_{R}(\Leftrightarrow)$. A temperature gradient is imposed on the column by maintaining the top at a temperature. $T_{T}$, and the bottom at temperature $T_{8 .} T_{\alpha}(=)$ is usually a linear temperature gradient situation with the gradient matched approximately to that established in the test stack. However. an isothermal guard with $T_{s}(\xi)$ equal to the average temperature of the specimen may also be used. An unguarded system is not recommended due to the potential very large errors paricularly at elevated temperatures (1). Ai steady state, the temperature gradients along the sections are calculated from measured temperatures

\footnotetext{
${ }^{7}$ The boldiace numbers in parentheses refer to a liss of references at the end of this test method.
} 


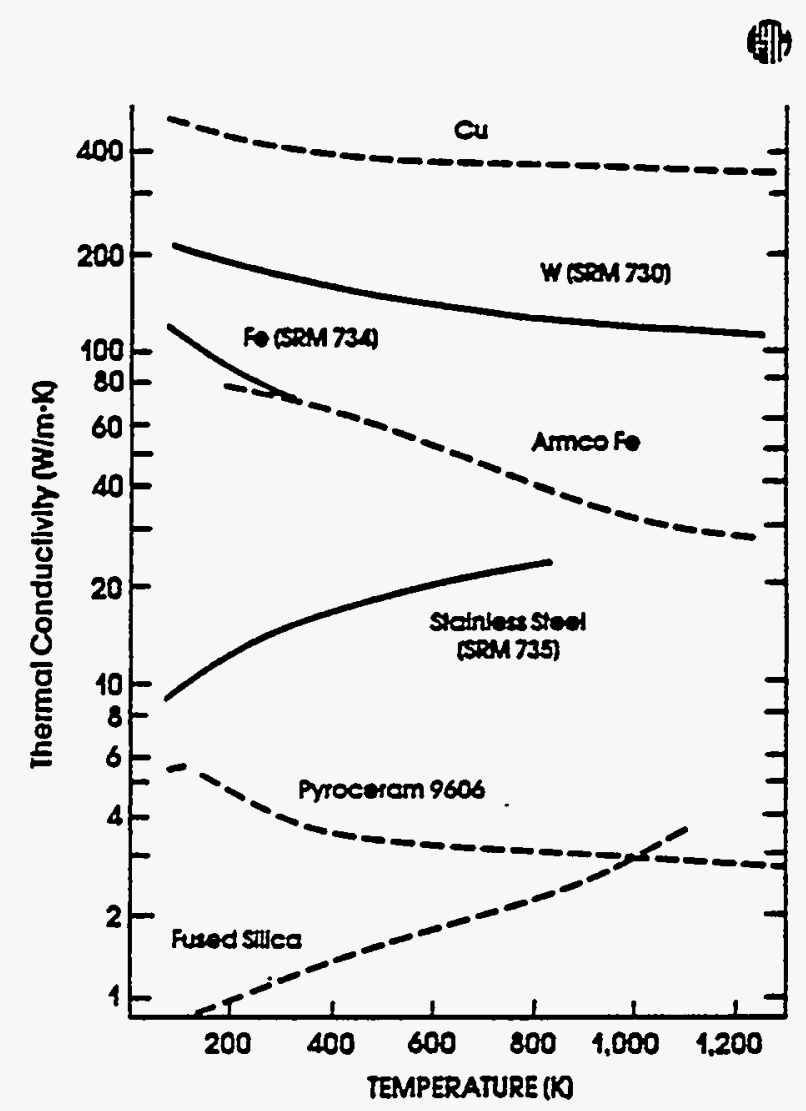

Note-The matenal salected for the meter bars should hrve a themal conducturty as near as possible to the thermal conctuctivity of the unitnown.

FIG. 2 Approximate Values for the Thermal Conductivity of Several Possible Reterence Materials for Meter Bars

along the two meter bars and the specimen. The value of $\lambda_{s}$ ( $\lambda_{S}$ as uncorrected for heat shunting) can then be determined using the following where the notation is shown in Fig. 1:

$$
\lambda_{3}{ }^{\prime}=\frac{\left(Z_{4}-Z_{3}\right)}{\left(T_{4}-T_{3}\right)} \frac{\lambda_{31}{ }^{\prime}}{2} \frac{\left(T_{3}-T_{1}\right)}{\left(Z_{2}-Z_{3}\right)}+\left[\frac{\lambda_{4}^{2}}{2} \frac{\left(T_{6}-T_{3}\right)}{\left(Z_{6}-Z_{3}\right)}\right]
$$

This is a highly idealized situation, however, since it assumes no heat exchange between the column and insulation at any position and uniform heat transfer at each meter barspecimen interface. The errors caused by these two assumptions vary widely and are discussed in Section 9. Because of these two effects, restrictions must be placed on this test method if the desired accuracy is to be achieved.

\section{Significance and Use}

5.1 The comparative method of measurement of thermal conductivity is especially useful for engineering materials including ceramics. polymers, metals and alloys, refractories, carbons. and graphites including combinations and other composite forms of each. It is capable of generating relatively accurate data on specimens that are too small (or possibly have an unusual shape) to accept heater and heat sink designs required by absolute methods.

5.2 Proper design of a guarded-longitudinal system is difficult and it is not practical in a method of this type to try to establish details of construction and procedures to cover all contingencies that might offer difficulties to a person

\section{E 1225}

without technical knowiedge concerning theory of heat : temperature measurements, and general testing prac. Standardization of this test method is not intended to res in any way the future development by research worke: new or improved methods or improved procedures $t$ ever, new or improved techniques must be thoroughly te and requirements for qualifying an apparatus are outline Section 10.

\section{Requirements}

6.1 Meter Bar Reference Materials:

6.1.1 Reference materials or transfer standards : known thermal conductivities must be used for the $\mathrm{m}$ : bars. Since the minimum measurement error of the meth is the uncertainty in $\lambda_{M}$, it is preferable to use standa available from a national standards laboratory. Other $\pi$ : ence materials are available because numerous measi ments of $\lambda$ have been made and general acceptance of values has been obtained. Table $I$ lists the currently avaita recognized reference materials including those availa from National Institute of Standards and Technology. Fis shows the approximate variation of $\lambda_{M}$ with temperatiri

6.1.2 Table 1 is not exhaustive and other materials may used as references. The reference material and the source $\lambda_{M}$ values shall be stated in the report.

6.1.3 The requirements for any reference material cludes stability over the temperature range of operatic compatibility with other system components, reasonat cost, ease of thermocouple attachment, and an accurate known thermal conductivity. Since heat shunting errors fo: specific $\lambda_{I}$ increase as $\lambda_{M} / \lambda_{s}$ varies from unity, (1) [ reference which has a $\lambda_{M}$ nearest to $\lambda_{s}$ should be used for $t$ meter bars.

6.1.4 If a sample has a $\lambda_{s}$ between two reference materiat the reference with the higher $\lambda_{M}$ should be used to reduce tt total temperature drop along the column.

\subsection{Insulation Materials:}

6.2.1 A large variety of powder, particulate, and inx materials exist for reducing both radial heat flow in th column-guard annulus and surrounds and heat shuntir along the column. Several factors must be considered duris selection of the most appropriate insulation. The insulatio: must be stable over the anticipated temperature range, har a low $\lambda_{l}$, and be easy to handle. In addition, the insulatio: should not contaminate system components such as to temperature sensors, it must have low toxicity, and it shouk not conduct electricity. In general, powders and particulate are used since they pack readily. However, low density fibe blankets can also be used.

6.2.2 Some candidate insulations are listed in Table 2

6.3 Temperature Sensors:

6.3.1 There shall be a minimum of two temperature sensors on each meter bar and two on the specimen. Whenever possible, the meter bars and specimen should exi contain three sensors. The extra sensors are useful it confirming linearity of temperature versus distance along the column or indicating an error due to a temperature sensor decalibration.

6.3.2 The type of temperature sensor depends on the system size, temperature range. and the system environmes: as controlled by the insulation, meter bars, specinen. ad 
TABLE 1 Reference Materials For Use as Meter Bars

\begin{tabular}{|c|c|c|c|c|}
\hline Materal & Temperature Renge $(K)$ & $\begin{array}{l}\text { Percentage } \\
\text { Uncertanty } \\
m \lambda(=x)\end{array}$ & $\lambda_{\omega}(W / m \cdot K)$ & Matenal Source \\
\hline Secroprtic Iron SAM 734 & To 1000 & 2 & $A$ & NISTA \\
\hline Tungten SRM 730 & $\begin{array}{l}4 \text { to } 300 \\
300 \text { to } 2000 \\
>2000\end{array}$ & $\begin{array}{l}2 \\
205 \\
5108\end{array}$ & $\lambda_{m}$ Dependent on TA & NISTA \\
\hline Nestente Staniess SRM 735 & 4101200 & $<5$ & $\lambda_{N}=1.22 T^{\circ} 02 T>200 K^{4}$ & NIST^ \\
\hline ran & 80 to 1200 & 2 & 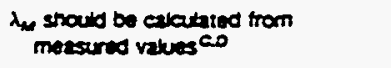 & $\cdots$ \\
\hline $\operatorname{cosen}$ & SO to 1250 & $<$ & $\begin{array}{c}\lambda_{N}=416.3-0.05904 T+7.087 \\
\times 10^{7} \pi^{3 e}\end{array}$ & manufacturer \\
\hline Proourem Code 9606 & 90101200 & $\ldots$ & $\approx .0$ & corame glass \\
\hline fused Srica" & 1300 & $\operatorname{lis}_{0} 10900 \mathrm{~K}$ & 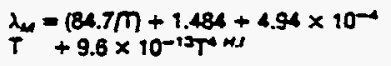 & grass \\
\hline $\operatorname{Arcx} \pi 740$ & 90 to 600 & 6 & . & glass \\
\hline
\end{tabular}

¿ Mapond Institute of Standares and Tectrnology. Washington. O.C. 20234. See Spocivl Pubteatons $260-52$ and 260-46.

- Hust J. G. Cryogenves Divison: NBS. Boulder. Covorado 80302.

EFuterson W., et al. Physies Roview 167. p. 765. (1968).

Dudes C. F.. Journal of Terting and Eveluation. ASTM 1 (5). 422 (1973).

(Moore, J. P.. Graves. R. S. and MeElroy. D. L. Canadien Joumal of Physics. 45. 3849 (1967).

-Thermal Conducturty of Selected Materats." Report NSROS-NBS 8. Natonal Bureau of Standards. 1966.

aL C. Hulstom. R. P. Tye, and S. E. Sinth. Themel Conduetrity 19. Ed. D. W. Yarorougn. Pienum Press. New York. in Course of Publeation (see also High Temperaturotigh Pressures. 17. 707. 1985.

" Nbove $700 \mathrm{~K}$ a large fraction of heat conduction in fused strea will be by radiation and the actud effective values may depend on the errutrances of beundtang surtaces motion bas size.

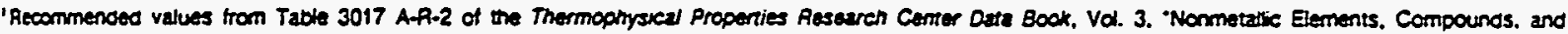
vartures, Purdue Unversity, Lafayette, Inctana.

TABLE 2 Suitable Themal Insulation Materials

\begin{tabular}{|c|c|c|c|}
\hline \multirow{2}{*}{ Matena' } & \multicolumn{3}{|c|}{ Typieal Therma Concucerviny $(\mathrm{W} / \mathrm{m} \cdot \mathrm{K})$} \\
\hline & $300 \mathrm{~K}$ & $800 \mathrm{~K}$ & $1300 K$ \\
\hline \multicolumn{4}{|l|}{ Mared Powders } \\
\hline Dusomacoous Earth & 0.053 & 0.10 & 0.154 \\
\hline Buoded Alumna & 0.21 & 0.37 & 0.41 \\
\hline Buodied Zroonia & 0.19 & 0.33 & 0.37 \\
\hline Vemafite & 0.07 & 0.16 & $\ldots$ \\
\hline Perte & 0.050 & 0.17 & $\ldots$ \\
\hline \multicolumn{4}{|l|}{ Burtets and Felts } \\
\hline Ahmosicate $60-120 \mathrm{~kg} / \mathrm{m}^{3}$ & 0.044 & 0.13 & 0.33 \\
\hline Zreonu $60-90 \mathrm{~kg} / \mathrm{m}^{3}$ & 0.039 & 0.09 & 0.25 \\
\hline
\end{tabular}

Al materals fisted can be used up to the $1300 \mathrm{~K}$ Ent of the comparauve unguand except where noted.

po within the system. Any sensor possessing adequate ucuracy may be used for temperature measurement (2) and be used in large systems where heat flow perturbation by the temperature sensors would be negligible. Thermocouples are comally employed. Their small size and the ease of atwxhing them are distinct advantages.

6.3.3 When thermocouples are employed they should be fibricated from wires which are $0.1 \mathrm{~mm}$ diameter or less. A consiant temperature reference shall always be provided for all cold junctions. This reference can be an ice-cold slurry (j). a constant temperature zone box or an electronic ice point reference. All thermocouples shall be fabricated from vither calibrated thermocouple wire (4) or from wire that has been centified by the supplier to conform with tables from E 230 to within the special limits of error given in Table 1 of E 230. E 230 should be consulted for information regarding thermocouple selection.

6.3.4 Thermocouple attachment is important to this techrique in order to ensure that reliable temperature measurs- ments are made at specific points. The various techniques are illustrated in Fig. 3. Intrinsic junctions can be obtained with metals and alloys by welding individual thermo-elements to the surfaces (Fig. 3a). Butt or bead welded thermocouples junctions can be rigidly attached by peening. cementing. or welding in fine grooves or small holes (Figs. 3b to $3 \mathrm{~d})$.

6.3.5 In Fig. 3b. the thermocouple resides in a radial slot. and in Fig. $3 c$ the thermocouple is pulled through a radial hole in the material. When a sheathed thermocouple or a thermocouple with both thermoelements in a two-hole electrical insulator is used. the thermocouple attachment shown in Fig $3 d$ can be used. In the latter three cases. the thermocouple should be thermally connected to the solid surface using a suitable glue or high temperature cement. All four of the procedures shown in Fig. 3 should include wire tempering on the surfaces. wire loops in isothermal zones. thermal wire grounds on the guard, or a combination of all three (5).

6.3.6 Since uncertainty in temperature sensor location leads to large errors. special care must be taken to determine the correct distance between sensors and to calculate the possible error resulting from any uncertainty.

6.4 Reduction of Contact Resistance:

6.4.1 This test method requires uniform heat transfer at the meter bar to specimen interfaces whenever the temperature sensors are within a distance equal to $r_{A}$ from an interface (6). This requirement necessitates a uniform contact resistance across the adjoining areas of meter bars and specimens. This is normally attained by use of an applied axial load in conjunction with a conducting medium at the interfaces. Measurements in a vacuum environment are not recommended unless the vacuum is required for protection purposes. 


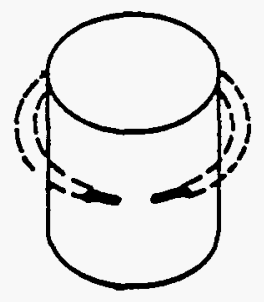

3 .

Intrnsx wex wth separate temperature elements maded to spocinen or meter burs so that signet is trough the matend.

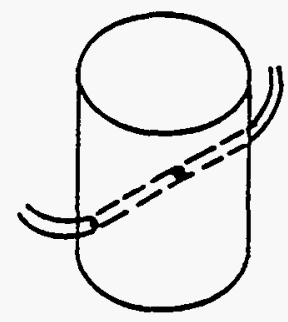

$3 c$

Small radial hole dniled trough the specomen or meter by and non-insulated (permited if the matenal is an etectncel insulater) of nsulated thermocouple pulled through the hole.

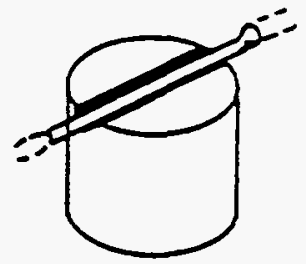

30

Raciel stots on the fat surtaces to hold a bere wire or ceremc neter themocouple senser the mity be bonced anto stot.

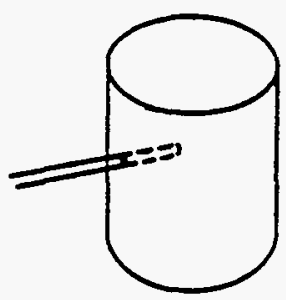

$3 d$

Smal Rediey hole orited part way trough the specinen of meter bor ar therinocouple pursed mo the hols.

NoTE- In all cases the thermotements should be themaly tempered and/or themaly grounded on the guard to minmize temperature measurement errors of br. flow into of out of the not junction.

FIG. 3 Thermocouple Attachments

6.4.2 For the relatively thin specimens normally used for materials having a low thermal conductivity the temperature sensors must be mounted close to the surface and in consequence the uniformity of contact resistance is critical. In such cases. a very thin layer of a compatible highly conductive fluid, paste, soft metal foil, or screen shall be introduced at the interfaces.

6.4.3 Means shall be provided for imposing a reproducible and constant load along the column with the primary purpose of minimizing interfacial resistances at meter barspecimen interfaces. Since the force applied to the column usually affects the contact resistance, it is desirable that this force be variable to ensure that $\lambda_{s}$ does not change with force variation. This force can be applied either pneumatically, hydraulically, by spring action, or by putting a dead weight on the column. The above load mechanisms have the advantage of remaining constant with change in column temperature. In some cases, the compressive strength of the specimen might be so low that the applied force must be limited to the dead weight of the upper meter bar. In this case. special care must be taken to limit error caused by poor contact by judicious positioning of temperature sensors away from any heat flow perturbation at the interfaces.

6.5 Guard Cylinder:
6.5.1 The specimen-meter bar column shall be enciose with a guard tube or pipe normally of right circular sye metry. This guard cylinder can be either a metal or a cerar but its inside radius should be such that the ratio $r_{B} / r_{A}$. be between 2.0 and 3.5 (1). This guard cylinder shall coote. at least one heater for controlling the temperature proife along the guard.

6.5.2 The guard shall be constructed and operated so the the temperature of the guard surface is either isothermal ax equal to the approximate mean temperature of the spenner or preferably have an approximately linear profile with th top and bottom ends of the guard matched to corresponies positions along the column. In each case. at least thri temperature sensors shall be attached to the guard at know. positions to measure the temperature profile.

\subsection{System Instrumentation:}

6.6.1 The combination of temperature sensor and the instrument used for measuring the sensor output shall bx adequate to ensure a temperature measurement precisios o $\pm 0.04 \mathrm{~K}$ and an absolute error less than $\pm 0.5 \%$.

6.6.2 Instrumentation for this technique shall be adeque to maintain the required temperature control and mearr. all pertinent output voltages with accuracy commensurat with the system capability. Although control can be manial 
I lechnique of this general description can be automated so that a computer carries out all the control functions. acquires Ill pertinent voltages. and calculates the thermal conducusity (7).

\section{- Sampling and Conditioning Test Specimens}

1.1 Test Specimens-This test method is not restricted to a particular geometry. General practice is to use cylindrical if square cross-sections. The conduction area of the specmen and reference samples must be the same to within $1 \%$ ixe. Vole 3) and any difference in area shall be taken into scount in the calculations of the result. For the cylindrical infiguration. the radii of the specimen and meter bars must uree 10 within $\pm 1 \%$ and the specimen radius, $r_{A}$, must be weh that $r_{B} / r_{A}$ is between 2.0 and 3.5. Each flat surface of ine specimen and reference must be flat with a surface finish qual to or better than $32 \sqrt{ }$ and the normal to each end shall $\tau$ parallel with the specimen axis to within $\pm 10 \mathrm{~min}$.

inte 3-In some enses this requirement is not necessary. For :umple. some appsartus might consist of meter bars and specimen with Thalues of $\lambda_{1,}$ and $\lambda_{s}$ so that thermal shunting errors would be small ir long sections. These sections might be long enough to permit -mperature sensor attachment far enough away from the interfaces that - allow uas uniform. The specimen lengih should be selected based on . miderations of radius and thermal conductivity. When $\lambda_{M}$ is higher in the thermal conductivity of SRM 735 (stainless steed). long rommens uith length/ $r$, $\gg 1$ can be used. These long specimens permit $\because$ use of large distances between temperature sensors and this reduces $x$ percentage error from uncerainty in sensor posizion. When $\lambda_{y}$ inte than the thermal conductivity of SRM 735. the length must be :ilkied because uncerninty from heat shunting becomes too large.

7.2 Sampling and Conditioning-Unless specifically rejuired or prescribed. one representative specimen shall be mepared from the sample and no preconditioning underialen.

\section{Calibration and Verification}

8.I There are many situations that call for equipment wass before operations on unknown materials can be successiully accomplished. These include the following:

8.1.1 After initial equipment construction.

8.1.2 When the ratio of $\lambda_{M}$ to $\lambda_{S}$ is less than 0.3 or greater than 3 and it is not possible to match thermal conductance ialues.

8.1.3 When the specimen shape is complex or the specmen is inordinately small.

\$.l.4 When changes have been made in the system sometry,

8.1.5 When meter bar or insulation material other than tose lised in 5.1 and 5.2 are considered for use, and

8.1.6 When the apparatus has been to a high enough imperature to change the properties of a component such as ixmocouples sensitivity.

8. These tests shall be run by comparing at least two iference materials in the following manner.

8.II A reference material which has the closest thermal ronductivity to the estimated thermal conductivity of the Ahnown should be machined according to 6.1. and

8.2. The $\lambda$ of this specimen fabricated from a reference zulerial shall then be measured as described in Section 9 aung meter bars fabricated from another reference material inich has the closest $\lambda$ to that of the specimen. For example. technique tests might be made by measuring on Pyroceram 9606 using meter bars fabricated from SRM 735 stainless steel. If the measured thermal conductivity of the specimen disagrees with the value from Table $\downarrow$ after all corrections for heat exchange are applied. additional effor is required to find the error source(s).

\section{Procedure}

9.1 Where possible and practical, select the reference specimens (meter bars) such that the thermal conductance is of the same order as that expected for the test specimen. After instrumenting and installing the proper meter bars. the specimen should be instrumented similarly. It should then be inserted into the test stack such that it is at aligned between the meter bars with at least $99 \%$ of each specimen surface in contact with the adjacent meter bar and sof foil or other contacting medium. if any, used to reduce interfacial resistance. If the system must be protected from oxidation during the test or if operation requires a particular gas or gas pressure to control $\lambda_{1}$. the system should be pumped and purged. and the operating gas and pressure established. The predetermined force required for reducing the effects of non-uniform interfacial resistance should be applied to the load column.

9.2 Heaters at either end of the column should be energized (see Note 4 ) and adjusted until the $T$ differences between positions $Z_{1}$ and $Z_{2} . Z_{3}$ and $Z_{4 .}$ and $Z_{5}$ and $Z_{6}$ are between 200 times the imprecision of the $\lambda T$ measurements and $30 \mathrm{~K}$ and the specimen is at the average temperature desired for the measurement. Although the exact temperature profile along the guard is not important for $r_{B} / r_{-4} \geqslant 3$. the power to the guard heaters should be adjusted until the temperature profile along the guard. $\left.T_{f}=\right)$. is constant with respect to time to within $\pm 0.1 \mathrm{~K}$ and either:

9.2.1 Approximately linear so that $T_{f}(\Leftrightarrow)$ coincides with the temperature along the sample column at a minimum of three places including the temperature at the top sensor on the top meter bar, the bottom sensor on the bottom bar. and the specimen midplane: or

9.2.2 Constant with respect to $=$ to within $\pm 5 \mathrm{~K}$ and matched to the average temperature of the test specimen.

Note 4-These heaters can either be attached to the ends of the meter bars or attacined to a structure adjacent to the meter bar. The heaters can be powered with A.C. or D.C. Several difterent heater configurations are acceptable. The power to these heaters shall be steady enough to maintain shor term $T$ fluctuations less than $\neq 0.03 \mathrm{~K}$ on the meter bar thermocouple nearest the heater. These two heaters. in conjunction with the guard shell heater and the system coolant shall maintain long term temperature drift less than $=0.05 \mathrm{~K} / \mathrm{h}$.

9.3 After the system has reached steady state ( $T$ drift $<0.05 \mathrm{~K} / \mathrm{h}$ ), measure the output of all temperature sensors.

\section{Calculation}

10.1 Approximate Specimen Thermal Conductivity:

10.1.1 The outputs from the temperature sensors shall be converted to temperature and the apparent heat fow per unit area. $q^{\prime}$, in the meter bars shall be calculated using the following:

$$
q_{T}^{\prime}=\lambda_{M} \frac{T_{2}-T_{1}}{Z_{2}-Z_{1}}
$$




$$
q_{B}^{\prime}=\lambda_{M} \frac{T_{6}-T_{s}}{Z_{b}-Z_{s}} \quad \text { bottom bar }
$$

In each of these equations, the $\lambda_{\mu}$ value (see Note 5 ) to be inserted shall be obtained from the information of 6.1 for the average meter bar $T$. Although these two values, $q^{\prime} T$ and $q_{\text {B }}^{\prime}$ should agree with each other to within about $\pm 10 \%$ when heat exchange with the insulation is small, good agreement is not a sufficient condition (nor always a necessary condition) for low heat shunting error.

10.1.2 A value for the specimen thermal conductivity at temperature $\left(T_{3}+T_{4}\right) / 2$, as uncorrected for heat exchange with the insulation can then be calculated using the following:

$$
\lambda^{\prime}{ }_{S}=\frac{\left(q^{\prime} T^{+} q_{B}^{\prime}\right)\left(Z_{4}-Z_{3}\right)}{2\left(T_{4}-T_{3}\right)}
$$

This would be at an average specimen $T$ of $\left(T_{4}+T_{3} / 2\right)$. Calculate $l / r_{A}\left(T_{4}-T_{3} / r_{A}\right) . \lambda_{M} / \lambda_{5}$, and apply a correction for extraneous heat flow as described in 10.2 to adjust $\lambda^{\prime}$ to $\lambda$. Report all results according to Section 11 .

Note 5-This type calculation procedure actually requires only two $T$ sensors on each column section. In this ose. the thind sensor on wach secrion serves 25 a test for consistency of the other two. Some calculation procedures require more than the two sensors to obrain more knowledge about $d T / d Z$

\subsection{Corrections for Extraneous Heat Flou:}

10.2.1 Calculation of the specimen thermal conductivity by a simple comparison of temperature gradients in the meter bars to that in the specimen is less valid when the specimen or meter bars, or both, have low thermal conductivities relative to that of the insulation. The apparatus should be designed to minimize these errors and information on this has been enclosed as an adjunct. The deviation from uniform heat flow has been expressed as (1) follows:

$$
\boldsymbol{\gamma}=F_{\boldsymbol{z}} F_{\lambda}
$$

where $F_{g}$ is a function of system dimensions, and $F_{\lambda}$ is a function of $\lambda_{.1 \%} \lambda_{\%}$. and $\lambda_{S}(1)$. The $F_{g}$ term has a value between 2 and 3 for the ratio of guard radius to column radius specified for this system. The $F_{\lambda}$ term is shown in Fig. 4 as a function of $\lambda_{S} / \lambda_{1}$ for various values of $\lambda_{M} / \lambda_{I}$ for a linear guard. At high ratios of $\lambda_{M} / \lambda_{f}$ and $\lambda_{S} / \lambda_{l}$, corrections would not be necessary since the departure from ideal heat flow would be small. For example, the product of $F_{\lambda}$ and $F_{k}$ would be less than $0.10(10 \%)$ for all measurements where $\lambda_{1} / \lambda_{1}$ and $\lambda_{S} / \lambda_{1}$ are greater than 30 . If the value of $F_{s} / F_{2}$ is to be kept below $10 \%$. the ratios $\lambda_{M} / \lambda_{l}$ and $\lambda_{s} / \lambda_{l}$ are greater than 30 . If the value of $F_{\ell} / F_{\lambda}$ is to be kept below $10 \%$, the ratios. $\lambda_{w} / \lambda_{1}$ and $\lambda_{s} / \lambda_{1}$ must be within the dotted boundaries on Fig. 4.

10.2.2 Measurements on materials where the ratios of $\lambda_{11} / \lambda_{1}$ and $\lambda_{S} / \lambda_{1}$ do not fall within these boundaries shall be accompanied with corrections for extraneous heat flow, but these corrections can be determined in the following three different ways:

10.2.2.1 Use of analytical techniques as described by Didion (1) and Finn (8).

10.2.2.2 Using calculations from finite-difference or finite-element heat conduction codes. and

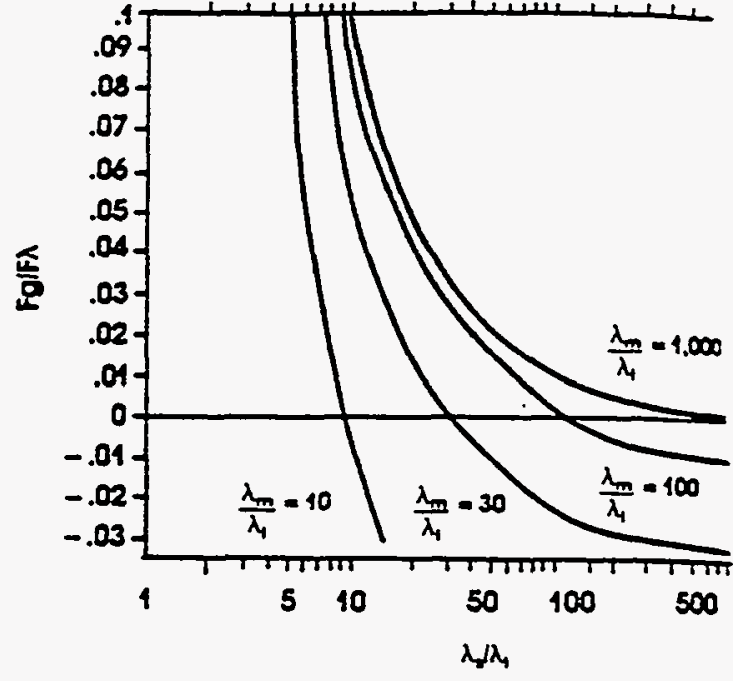

FiG. 4 Fractional Heat Exchange Batween the Mets Specimen Column and Surrounding Insulation as a Function o for Several Values of $\lambda_{d} / \lambda$

10.2.2.3 Determined experimentally by using severz erence materials or transfer standards of different the conductance as specimens. The procedure must be cautiously since all such specimens should have the sam: as the specimen with an unknown thermal conductivin have the same suriace finish.

\section{Report}

11.1 The report of the test results shall include following:

11.1.1 Complete specimen identification including st. and size,

11.1.2 Complete identification of insulation and soure $\lambda$, values, gas, and gas pressure,

11.1.3 Statements of thermocouple type, size, and attment procedure,

11.1.4 Complete listing of the geometrical dimension the system including $r_{A}, r_{B}$ specimen height, meter height, and distances between temperature sensors,

11.1.5 Column force,

11.1.6 Meter bar material and source of $\lambda_{M}$ values if or than those listed in Table 1 ,

11.1.7 Reference to the use of this test method si include a statement of the percentage variation of qualification results about the true value. For examr "thermal conductivity results on Pyroceram 9606 us: SRM 735 meter bars were within $\pm 4 \%$ of the accopi values for Pyroceram over the temperature range from: to $900 \mathrm{~K}$ "

11.1.8 Variations, if any, from this test method. If rest are to be reported as having been obtained by this meix then all requirements prescribed by this method shall be If Where such conditions are not met, the phase. "All reaul ments of this method have been met with the exceptis? ..." shall be added and a compiete list of the excep!no included. and 
11.1.9 Measured values of temperature and specimen thermal conductivity.

\section{Precision and Bias}

12.1 Determinate Errors-The determinate error of Eq. 1 is

$$
\begin{aligned}
& \left|\frac{\alpha_{s}^{\prime}}{\lambda_{s}}\right|=\left|\frac{\delta \lambda_{14}}{\lambda_{N}}\right|+\left|\frac{\delta\left(Z_{4}-Z_{3}\right)}{Z_{4}-Z_{3}}\right|+\left|\frac{\delta\left(T_{4}-T_{3}\right)}{T_{4}-T_{3}}\right| \\
& +\frac{\&\left(T_{2}-T_{1}\right)}{\left(Z_{2}-Z_{1}\right) C}+\frac{\left(T_{2}-T_{1}\right)\left(Z_{2}-Z_{1}\right)}{\left(Z_{2}-Z_{1}\right) C}+\frac{z\left(T_{6}-T_{3}\right)}{\left(Z_{6}-Z_{9}\right) C} \\
& +\frac{\&\left(T_{6}-T_{g}\right) \&\left(Z_{6}-Z_{g}\right)}{\left(Z_{6}-Z_{g}\right)^{2} C}
\end{aligned}
$$

where:

$$
C=\frac{T_{2}-T_{1}}{Z_{2}-Z_{1}}+\frac{T_{6}-T_{3}}{Z_{6}-Z_{9}}
$$

Usually the heat flows and temperature sensor spacings are esentially the same in the meter bars so that $C$ approaches a uumerical value of 2.

12.2 Example of Determinate Error:

12.2.1 Assumptions for a system where both meter bars and the specimen are of equal length is that the sensor parings are all $13 \mathrm{~mm}$ and $\lambda_{M}=\lambda_{S}$ :

$$
\begin{gathered}
\left|\frac{\delta \lambda_{34}}{\lambda_{3}}\right|=|0.003| \\
Z_{2}-Z_{1}-Z_{4}-Z_{3}-Z_{6}-Z_{9}=13 \mathrm{~mm}: \\
T_{2}-T_{1}-T_{4}-T_{3}-T_{6}-T_{3}=10 \mathrm{~K}:
\end{gathered}
$$

$$
\begin{gathered}
\alpha\left(Z_{2}-Z_{1}\right)-\alpha\left(Z_{4}-Z_{3}\right)-\alpha\left(Z_{6}-Z_{3}\right)=0.2 \mathrm{~mm}: \text { and } \\
\delta\left(T_{2}-T_{1}\right)-\alpha\left(T_{4}-T_{3}\right)-\delta\left(T_{6}-T_{3}\right)=0.04 \mathrm{~K}
\end{gathered}
$$

12.2.2 The maximum value of $\delta\left(Z_{2}-Z_{1}\right)$ etc. was approximated by assuming an uncertainty of $\pm 1 / 2$ (sensor diameter) at each temperature measurement position. Therefore, if the diameter of each sensor is $0.2 \mathrm{~mm}$, the uncertainty in the difference would be $\pm 0.2 \mathrm{~mm}$. The number for $\delta\left(T_{2}-T_{1}\right)$ etc. was calculated based on the sensor absolute accuracy.

12.2.3 With these values the fractional uncertainty in $\lambda^{\prime} s$, $\lambda^{\prime} S / \lambda^{\prime}$ s, will be !0.069! or $\pm 6.9 \%$.

12.3 Indeterminate Errors:

12.3.1 There are at least three other errors that can contribute to total system error and these are (1) nonuniform interfacial resistance, (2) heat exchange between the column and guard, and (3) heat shunting through the insulation around the column. These three errors must be minimized or appropriate corrections applied to the data if the desired accuracy is to be obtained.

12.3.2 The contributions from the last two errors can be determined approximately using results from appropriate experiments carried out at different levels of guard temperature to specimen stack temperature out of balance.

12.4 Overall-A recent international, interlaboratory round robin study also involving absolute methods (9) has shown that a precision of $\pm 6.8 \%$ can be attained over the temperature range 300 to $600 \mathrm{~K}$. Although no definite bias could be established these are indications that the values were the order of $2 \%$ lower than those obtained by absolute methods. This paper is on file at ASTM as a research report. 


\section{REFERENCES}

(1) Didion. D. A.. "An Analysis and Design of a Linear Guanded Cut-Bar Apparatus for Thermal Conductivity Measurements. "AD665789. January. 1968. available from the National Technieal Information Service. Springield. VA.

(2) Finch. D. 1., "General Principles of Thermoelastic Thermometry," in Temperature. I's Measurement and Control in Science and Indusiry: Vol. 3, Part 2 Secrion 1, Reinhold Publishing Corporauon.

(3) Caldwell. F. R.. "Temperatures of Thermocouple Reference Junctions in an lee Bar." J. Res. Nat. Bur. Std., 69C(2) (1965).

(4) ASME: PTC 19.3, Temperature Measurement, Part 3, p. 1232. 1974.

(5) Anderson. R. L., and Kollie, T. G. "Problems in High Temperature Thermometry." July, 1976, pp. 171-221.
(6) Fried. E. Thermal Conduativit; Vol 2. Tye. R. P. (ed), Acide Press, New York (1969).

(7) Morgan, M. T. and West, G. A. "Thermal Conductiviry of $t$ Rocks in the Bureau of Mines Standard Rock Site. Therm Conductivity. 16, Lassen, D. C. ed. Plenum Press. NY, NY, 198 pp 79-90.

(8) Fynn, D. R. Thermal Conductivity of Ceramice" Mecterex and Thermal Propenies of Ceramics. Special Publiarion $x$ Nacional Bureau of Standards, 1969.

(9) Hulstrom. L. C., Tye, R. P., and Smith, S. E. "Round-Rod Testing of Therral Conductivity Reference Materials," Them Conducivity. 19. Yarbrough. D. W. ed. Plenum Press. New Yox (See also High-Temp-High Pressures, Vol 17, p. 707, 1985). 